\title{
Application of Statistical Learning Theory to Plankton Image Analysis

\author{
Qiao $\mathrm{Hu}$
}

B.S., University of Science and Technology of China(1990)

M.S., University of Science and Technology of China(1993)

Submitted to the Joint Program in Applied Ocean Science and

Engineering

in partial fulfillment of the requirements for the degree of

Doctor of Philosophy

at the

MASSACHUSETTS INSTITUTE OF TECHNOLOGY and the

WOODS HOLE OCEANOGRAPHIC INSTITUTION June 2006

(C)Woods Hole Oceanographic Institution 2006. All rights reserved.

Author

Joint Program in Applied Ocean Science and Engineering

April 25, 2006

Certified by

Cabell S. Davis

Senior Scientist, WHOI

Thesis Supervisor

Certified by

Hanumant Singh

Associate Scientist, WHOI

Thesis Supervisor

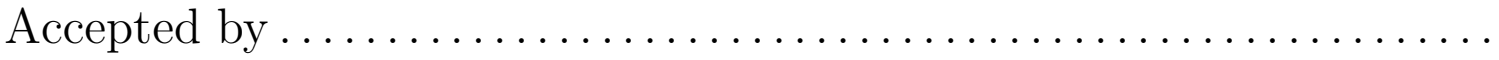

Henrik Schmidt

Chairman, Joint Committee for Applied Ocean Science and

Engineering

Accepted by

Lallit Anand

Chairman, Committee on Graduate Student, Department of Mechanical Engineering 


\title{
Application of Statistical Learning Theory to Plankton Image Analysis
}

by

\author{
Qiao Hu
}

\begin{abstract}
Submitted to the Joint Program in Applied Ocean Science and Engineering on April 25, 2006, in partial fulfillment of the

requirements for the degree of

Doctor of Philosophy
\end{abstract}

\begin{abstract}
A fundamental problem in limnology and oceanography is the inability to quickly identify and map distributions of plankton. This thesis addresses the problem by applying statistical machine learning to video images collected by an optical sampler, the Video Plankton Recorder (VPR). The research is focused on development of a real-time automatic plankton recognition system to estimate plankton abundance. The system includes four major components: pattern representation/feature measurement, feature extraction/selection, classification, and abundance estimation.

After an extensive study on a traditional learning vector quantization (LVQ) neural network (NN) classifier built on shape-based features and different pattern representation methods, I developed a classification system combined multi-scale cooccurrence matrices feature with support vector machine classifier. This new method outperforms the traditional shape-based-NN classifier method by $12 \%$ in classification accuracy. Subsequent plankton abundance estimates are improved in the regions of low relative abundance by more than $50 \%$.

Both the NN and SVM classifiers have no rejection metrics. In this thesis, two rejection metrics were developed. One was based on the Euclidean distance in the feature space for NN classifier. The other used dual classifier (NN and SVM) voting as output. Using the dual-classification method alone yields almost as good abundance estimation as human labeling on a test-bed of real world data. However, the distance rejection metric for NN classifier might be more useful when the training samples are not "good" ie, representative of the field data.

In summary, this thesis advances the current state-of-the-art plankton recognition system by demonstrating multi-scale texture-based features are more suitable for classifying field-collected images. The system was verified on a very large realworld dataset in systematic way for the first time. The accomplishments include developing a multi-scale occurrence matrices and support vector machine system, a dual-classification system, automatic correction in abundance estimation, and ability to get accurate abundance estimation from real-time automatic classification. The
\end{abstract}


methods developed are generic and are likely to work on range of other image classification applications.

Thesis Supervisor: Cabell S. Davis

Title: Senior Scientist, WHOI

Thesis Supervisor: Hanumant Singh

Title: Associate Scientist, WHOI 


\section{Acknowledgments}

First of all, I would like to thank my co-advisors, Cabell Davis and Hanumant Singh. They stand on my side with only occasional doubts. Even when I seemed have no way out. Cabell Davis was always there when I needed his guidance. His enthusiasm and "hands-on" approaches to science, both in the lab and during cruises, have been an inspiration to me. He is a great scientist and a good mentor. Hanumant Singh kept me on the track. He provided me the opportunity to share my work with fellow students. He is a really expert on underwater imaging.

I would like to thank the rest of my committee members, Jerome Milgram and George Barbastathis for their suggestions and useful criticism. I would like thank Carin Ashjian for serving as chair of my defense.

This work was supported by National Science Foundation Grants OCE-9820099 and Woods Hole Oceanographic Institution academic program.

I would like thank Marine Ecology Progress Series for publishing my thesis Chapter 5 and 6 and giving me permission to include them in my thesis. Thanks to editors to make these two chapters more readable.

I would like thank everyone in the Video Plankton Recorder group. Cabell Davis, Scott Gallager, Carin Ashjian, Xiaoou Tang, Philip Alatalo, Andrew Girard and all the others collected the images I used in my thesis. Cabell Davis and Philip Alatalo taught me how to classify these images manually.

I would like thank everyone in Seabed AUV group. Hanumant Singh, Oscar Pizarro, Ryan Eustice, Christopher Roman, Michael Jakuba and Anna Michel gave me lots of comments and suggestions on my thesis work.

I would like thank everyone at WHOI Academic programs office. John Farrington, Judith McDowell, Julia Westerwater, Marsha Gomes and Stacey Brudno Drange made my long journey so wonderful and enjoyable.

I would like thank everyone who are kind enough to read my thesis draft. Judith Fenwick, Colleen Petrik, Gareth Lawson and Sanjay Tiwari read part or whole thesis.

I would like thank all my friends: Xiaoou Tang, Guangyu Wu, Weichang Li, 
Wenyu Luo and Jinshan Xu, Zao Xu and Gareth Lawson. I had such a memorable time with them.

Last but not least, I would like thank my family. My wife Xingwen Li and my daughter Daisy Xuyuan Hu make such a warmth home for me. Their love, constant support and confidence gave me the great strength to break through all the difficulties. I thank my parents and my sisters for their understanding and patience. 


\section{Contents}

1 Introduction $\quad 25$

1.1 Motivation ......................... 26

1.2 Statistical pattern recognition . . . . . . . . . . . . 26

1.2.1 Features ....................... 27

1.2.2 Statistical learning theory . . . . . . . . . . . . . . . . . 29

1.3 An overview of related work . . . . . . . . . . . . . 30

1.4 Data . . . . . . . . . . . . . . . . . . . 32

1.5 Thesis overview . . . . . . . . . . . . . . . . . 33

2 Data Acquisition $\quad 39$

2.1 Water column plankton samplers . . . . . . . . . . . . . . 39

2.2 Video Plankton Recorder . . . . . . . . . . . . . . . . . . 42

2.3 Focus Detection . . . . . . . . . . . . . . . . . . 44

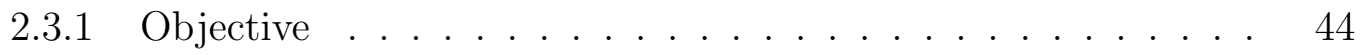

2.3.2 Method .................... 46

2.3.3 Algorithms ..................... 47

2.3.4 Result ....................... . . 48

2.4 Conclusion . . . . . . . . . . . . . . . . . 52

3 Classification Method: Analysis and Assessment 53

3.1 System overview . . . . . . . . . . . . . . . . . 54

3.1.1 Artificial Neural Networks . . . . . . . . . . . . . . . 54

3.1.2 Learning vector quantization neural network classifier . . . . . 54 
3.1.3 Principal component analysis . . . . . . . . . . . 55

3.1 .4 Feature extraction . . . . . . . . . . . 56

3.1.5 Classification performance estimation . . . . . . . . 56

3.2 Assessment Result . . . . . . . . . . . . . . . . . . . . . . . . 58

3.2.1 Classifier complexity vs. classifier performance . . . . . . . 58

3.2.2 Feature length versus classification performance . . . . . . 58

3.2.3 Learning curve - numbers of training samples versus classifier performance . . . . . . . . . . . . . . 60

3.2.4 Initial neuron position versus presentation order of training samples . . . . . . . . . . . . . . . 63

3.2.5 Training samples effect . . . . . . . . . . . . . . 64

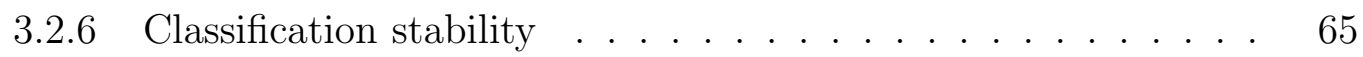

3.3 Two-pass classification system . . . . . . . . . . . . . 66

3.3 .1 Decision rules . . . . . . . . . . . . . . . . . . . . 68

3.3 .2 Implementation . . . . . . . . . . . . . . . . . . 71

3.3 .3 Results . . . . . . . . . . . . . . . . . . . . . 71

3.3 .4 Discussion . . . . . . . . . . . . . . . . . . 73

3.4 Statistical correction method . . . . . . . . . . . . 75

3.4 .1 Method . . . . . . . . . . . . . . . 75

3.4 .2 Result . . . . . . . . . . . . . . . . 76

3.5 Distance rejection metric for LVQ-NN classifier $\ldots \ldots \ldots \ldots \ldots$

3.5.1 Distance rejection system $\ldots \ldots \ldots \ldots \ldots$

3.5.2 Result and discussion . . . . . . . . . . . . . 78

3.6 Conclusion . . . . . . . . . . . . . . . . . . . . . . . . . . 83

4 Pattern Representation/Feature Measurement $\quad 85$

4.1 Pattern representation methods . . . . . . . . . . . 86

4.1.1 Shape-based methods . . . . . . . . . . . . . . . . 86

4.1.2 Texture-based methods . . . . . . . . . . . . . . . . . 90

4.1 .3 Other Methods . . . . . . . . . . . . . . . 95 
4.2 Feature extraction and classification . . . . . . . . . . 97

4.3 Results and discussion . . . . . . . . . . . . . . . 100

4.3 .1 High order moment invariants . . . . . . . . . . . . 103

4.3.2 Radial Fourier descriptors vs. complex Fourier descriptors . . 105

4.3 .3 Co-occurrence matrices . . . . . . . . . . . . . . . . 107

4.3.4 Edge frequency . . . . . . . . . . . . . . . 110

4.3.5 Run length . . . . . . . . . . . . . . . . 111

$4.3 .6 \quad$ Pattern spectrum . . . . . . . . . . . . . . . . . 112

4.3.7 Wavelet transform . . . . . . . . . . . . . . . . 112

4.4 Conclusion . . . . . . . . . . . . . . . . . . . . . . . . . . . 114

5 Co-Occurrence Matrices and Support Vector Machine 115

5.1 Co-Occurrence Matrices . . . . . . . . . . . . . . . . 116

5.2 Support Vector Machines . . . . . . . . . . . . . . . . . . 116

5.3 Feature Extraction and Classification . . . . . . . . . . . . 118

5.4 Classification results . . . . . . . . . . . . . . . . . . . . . . . . . 119

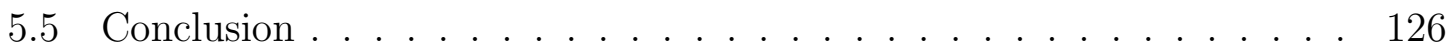

6 Dual classification system and accurate plankton abundance estimation

6.1 Dual classification system description . . . . . . . . . . . . 130

6.1 .1 Pattern representations . . . . . . . . . . . . . 130

6.1 .2 Feature extraction . . . . . . . . . . . . . . . 132

6.1 .3 Classifiers . . . . . . . . . . . . . . . . . . 133

6.1.4 Dual classification system . . . . . . . . . . . . . . 134

6.1.5 Classification performance evaluation and abundance correction 136

6.2 Classification results . . . . . . . . . . . . . . . . . . . 138

6.3 Conclusion . . . . . . . . . . . . . . . . . . . . . . 147

$\begin{array}{lll}7 & \text { Conclusions and future work } & 149\end{array}$

7.1 Summary of major contributions _ . . . . . . . . . . 150 
7.2 Future research directions . . . . . . . . . . . . . . . . . . 153 


\section{List of Figures}

1-1 Schematic diagram of the pattern recognition system. . . . . . . . . . 28

1-2 Example VPR images of copepods, rod-shaped diatom chain, Chaetoceros socialis colonies and the "other" category. Fifty randomly selected samples are shown here. . . . . . . . . . . . . . . . . . . . 34

1-3 Example VPR images of Chaetoceros chains and marine snow. Fifty randomly selected samples are shown here. . . . . . . . . . . . . . . . 35

1-4 Example VPR images of hydroid medusae. Fifty randomly selected samples are shown here. . . . . . . . . . . . . . . . . . . 36

2-1 Video Plankton Recorder system with underwater and shipboard components. The VPR is towyoed at ship speeds up to $5 \mathrm{~m} / \mathrm{s}$, while video is processed in real-time on board. . . . . . . . . . . . . . . . 43

2-2 The graphical user interface of real time focus detection program. . . . . 45

3-1 Classification performance with respect to classifier complexity. . . . . 59

3-2 Training and test accuracy with respect to classifier complexity. . . . 59

3-3 Classification performance as a function of feature length for each taxon. 60

3-4 Training and test accuracy with respect to feature length. . . . . . . . 61

3-5 Classification performance as a function of training sample size for each

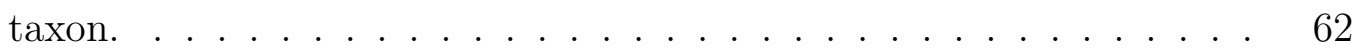

3-6 Training and test accuracy with respect to training sample size. . . . 63 
3-7 Comparison between the random initial position of neurons and random order of presentation order of training samples. IP1 - different initial position of neurons, random representation order of training samples; IP2 - same initial position of neurons, random representation order of training samples . . . . . . . . . . . . . . .

3-8 Comparison of different training samples effect on classfication performance. TS1 - different sets of training samples, leave-one-out method; TS2 - different sets of training samples, holdout method; TS3 - single set training samples, holdout method . . . . . . . . . . . .

3-9 Mean, upper and lower limit of $95 \%$ confidence interval of abundance estimates from LVQ-NN classifiers and that of manually sorted results. Time series abundance plots along the tow path are shown for 6 dominant taxa. Data were first binned in 10 second time intervals, and a one-hour smoothing window was applied to the binned data. . . . . .

3-10 Illustration of the three most popular decision rules. $x_{M L}^{*}$ - maximum likelihood decision rule, $x_{M A P}^{*}$ - maximum a posteriori decision rule, $x_{M M}^{*}-\operatorname{minimax}$ decision rule . . . . . . . . . . . . . . 70

3-11 Schematic diagram of two pass classification system . . . . . . . . 72

3-12 Comparison of two automatic classification systems with human expert classified results. Time series abundance plots along the tow path are shown for 6 dominant taxa. Data were first binned in 10 second time intervals, and a one-hour smoothing window was applied to the binned

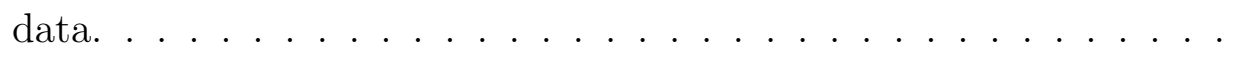

3-13 Comparison of automatic classification systems with/without statistical correction to human expert classified results. Time series abundance plots along the tow path are shown for 6 dominant taxa. Data were first binned in 10 second time intervals, and a one-hour smoothing window was applied to the binned data. . . . . . . . . . 77

3-14 Schematic diagram of distance rejection classification system . . . . 79 
4-1 Mean and standard deviation of classification accuracy from different feature presentation methods for each taxon. The abbreviations are as follows: MI - moment invariants, FD - Fourier descriptors, SS - shape spectrum, MM - morphological measurements, CM - co-occurrence matrices, RL - run length, EF - edge frequency, PS - pattern spectrum, WT - wavelet transform. It clearly shows the jump between shapebased features and texture-based features. The pattern spectrum and wavelet transform methods are between shape-based and texture-based methods, their performances lie in between these two group of methods.102

4-2 Illustrates the problem of non-uniform illunimation on segmentation. (a) the original image, (b) gradient correction of (a), (c) segmentation of (a), (d) segmentation of (b), (e) contour of the largest object from

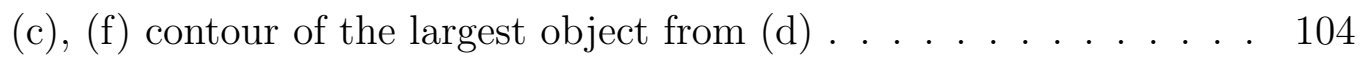

4-3 Mean and standard deviation of classification accuracy for moment invariants of different orders for each taxon. MI3-7 stands for moment invariants up to order 3-7, which correspond to feature length of 7,12 , 18, 24, and 33 respectively. where MI3 is equivalent to Hu's moment invariants. There is no benefit to using high order moment invariants in this dataset. . . . . . . . . . . . . . . . . . . . . 105

4-4 Illustrates the problem of using a radius-vector function to encode a non-star-shaped plankton image. (a) the original image, (b) the contour of the object, (c) the radius-vector function from the contour model of (b), (d) the recovered contour of the object based on radiusvector function (c) with assumption that the object is star-shaped. . . 106

4-5 A comparison between radial Fourier descriptors (RFD) and complex

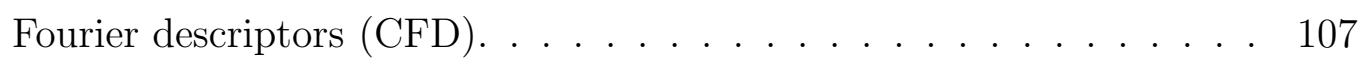


4-6 Mean and standard deviation of classification accuracy for co-occurrence matrices of different multi-scale levels for each taxon. The abbreviations CM1-6 stand for co-occurrence matrices of multi-scale levels from 1 to 6 , which correspond to feature length of $16,32,48,64,80$, and 96 respectively. The classification accuracy first rises sharply with an increase of multi-scale levels, and reaches top performance with 3-4 multi-scale levels. The performance then drops down slightly as more multi-scale levels are included in the feature set. . . . . . . . . . . . . 108

4-7 A comparison between raw co-occurrence matrices (RCM) and statistics of co-occurrence matrices (SCM). There is little difference between $\mathrm{SCM}$ and RCM. . . . . . . . . . . . . . . 109

4-8 Virtual support vector machine test on the co-occurrence matrices method. SCM - statistics of co-occurrence matrices, CCM - statistics of co-occurrence matrices from original image and its complement, RCM - statistics of co-occurrence matrices from original image and resized images of 0.8 and 1.2. No accuracy gain is obtained by adding virtual samples in the training set. . . . . . . . . . . . .

4-9 A comparison of edge frequency features, where EF1 is EF spectrum with linear distance interval from 1 to 40 pixels and two directions formula (horizontal \& vertical, and diagonals), EF2 is EF with 7 exponential distance interval from 1 to 64 and two directions formula (horizontal \& vertical, and diagonals), EF3 is EF with 7 exponential distance interval from 1 to 64 and four directions formula (horizontal, vertical and two diagonals). It is clear that exponential distance interval works better than the linear distance interval, and four direction formula works better than two direction formula. . . . . . . . . 
4-10 Comparison of run length methods. RL1 - run length statistics proposed by Galloway, 5 statistics from each run length matrix, total 20 features for 4 directions. RL2 - extended run length statisitcs by Chu et al., and by Dasarathy and Holder. 11 statistics from each run length matrix, total 44 features for 4 directions. The extended features give a slight better performance for all the taxa. . . . . . . . . . .

4-11 Comparison between two implementations of pattern spectrum. PS1 PS by Vincent, linear and pseudo opening and closing spectra, each has 40 elements, total feature length of 160. PS2 - PS modified from Meijster and Wilkinson, horizontal and vertical line opening and closing spectra, and area opening and closing spectra, each has 40 elements, total feature length of 240. PS1 outperforms PS2 on all the taxa except marine snow. . . . . . . . . . . . . . . . . .

4-12 Multi-scale level test for wavelet transform features. WL1-7 stands for features from wavelet transform with multi-scale level from 1 to 7.

5-1 Comparison of 2 automated classifier with human expert classified results for 6 dominant taxa along the tow timescale. CSF-NN, combined shape-based features and neural network; COM-SVM, co-occurrence features and support vector machine. The data are first binned into 10 second time intervals. A 1 hour smoothing window is applied to the binned data. . . . . . . . . . . . . . . . . .

5-2 Comparison of 2 automated classifier with human expert classified results for 6 dominant taxa along the tow timescale. CSF-NN, combined shape-based features and neural network; CSF-SVM, combined shapebased featuresand support vector machine. The data are first binned into 10 second time intervals. A 1 hour smoothing window is applied to the binned data. . . . . . . . . . . . . . . 
5-3 Reduction in the relative abundance estimation error rate between COM-SVM and CSF-NN, and between CSF-SVM and CSF-NN. The positive value indicates that COM-SVM/CSF-SVM is better than CSF$\mathrm{NN}$, while the negative value indicates COM-SVM/CSF-NN is worse than CSF-NN. . . . . . . . . . . . . . . . . . . 125

6-1 Schematic diagram of dual-classification system. LVQ: learning vector quantization; NN: neural netowork; SVM: support vector machine .

6-2 Automatically classified images: comparison of results for $(\mathrm{A}, \mathrm{C})$ dualclassification system and (B,D) single neural network classifier. The first 25 images classified as (A,B) copepods and (C,D) Chaetoceros socialis by the dual-classification system and LVQ-NN classifier are shown. For taxa having relatively high abundance, such as copepods, both systems yield very similar results (21 out of 25 were the same). In contrast, for taxa having relatively low abundance, such as lowabundance regions of $C$. socialis, the dual-classification system has much higher specificity (fewer false alarms). . . . . . . . . . .

6-3 Comparison of dual-classification, and manually corrected single NN classification with human expert classified results for 6 dominant taxa along the tow timescale. The data are first binned into 10 second time intervals. A 1 hour smoothing window is applied to the binned data. .

6-4 Comparison of 3 automated classifier with human expert classified results for 6 dominant taxa along the tow timescale. CSF-NN, combined shape-based features and neural network; CSF-SVM, combined shapebased features and support vector machine. The data are first binned into 10 second time intervals. A 1 hour smoothing window is applied to the binned data. . . . . . . . . . . . . . . 
6-5 Reduction in error rate between the dual- and single-classification systems for relative abundance estimation. Positive values indicate that the dual-classification outperforms other methods; negative values indicate the opposite. Dual: dual-classification system, CSF-NN: combined shape-based features and neural network; CSF-SVM, combined shape-based features and support vector machine. . . . . . . . . . . . 145

6-6 Relationship between specificity and relative abundance for different false alarm rates. Probability of detection is set to $70 \%$. As false alarm rates become smaller, the range in which the specificity closes to a constant becomes wider. The dual-classification system has substantially reduced the false alarm rates, so that the specificity of each taxon in the whole study area is close to a constant. This makes fully automatic correction possible. . . . . . . . . . . . 146 


\section{List of Tables}

2.1 Comparison of focus detection algorithms from AN9703, high magnification camera, video section 1 . The numbers are blob counts; probability of detection $P_{d}$ and probability of false alarm $P_{f}$ are given as

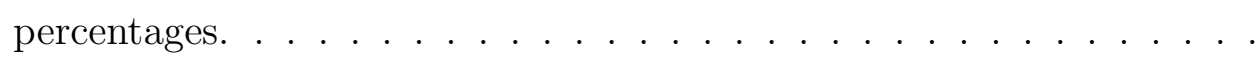

2.2 Comparison of focus detection algorithms from AN9703, high magnification camera, video section 2. The numbers are blob counts; probability of detection $P_{d}$ and probability of false alarm $P_{f}$ are given as

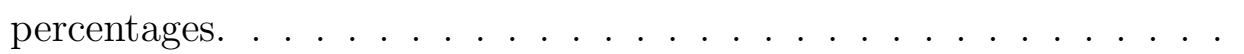

2.3 Comparison of focus detection algorithms from AN9703, high magnification camera, video section 1 after correction. The numbers are blob counts; probability of detection $P_{d}$ and probability of false alarm $P_{f}$ are given as percentages. . . . . . . . . . . . . . .

2.4 Comparison of focus detection algorithms from AN9703, high magnification camera, video section 2 after correction. The numbers are blob counts; probability of detection $P_{d}$ and probability of false alarm $P_{f}$ are given as percentages. . . . . . . . . . . . . . . .

2.5 Comparison of focus detection algorithms from HALOS, low magnification camera, video section 1. The numbers are the blob counts; probability of detection $P_{d}$ and probability of false alarm $P_{f}$ are given

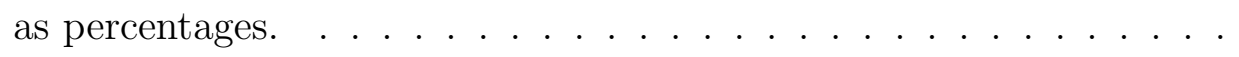


2.6 Comparison of focus detection algorithms from HALOS, low magnification camera, video section 2. The numbers are the blob counts; probability of detection $P_{d}$ and probability of false alarm $P_{f}$ are given

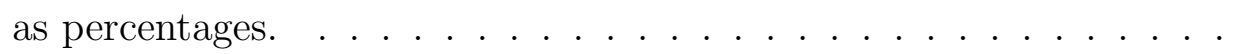

3.1 Confusion matrix of an LVQ-NN classifier with distance rejection metric using hold-out method. The classifier was trained without marine snow. Column and row heading are coded as: C1, copepod; C2, rodshaped diatom chains; C3, Chaetoceros chains; C4, Chaetoceros socialis colonies; C5, hydroid medusae; C6, marine snow; C6* "unknown". .

3.2 Confusion matrix of an LVQ-NN classifier with distance rejection metric using hold-out method. The classifier was trained without hydroid medusae. Column and row heading are coded as: C1, copepod; C2, rod-shaped diatom chains; C3, Chaetoceros chains; C4, Chaetoceros socialis colonies; C5, marine snow; C6, hydroid medusae; C6*, "unknown". 81

3.3 Confusion matrix of an LVQ-NN classifier with distance rejection metric using hold-out method. The classifier was trained without Chaetoceros socialos colonies. Column and row heading are coded as: C1, copepod; C2, rod-shaped diatom chains; C3, Chaetoceros chains; C4, hydroid medusae; C5, marine snow; C6, Chaetoceros socialis colonies; C6*, "unknown". . . . . . . . . . . . . . . . . .

3.4 Confusion matrix of an LVQ-NN classifier with distance rejection metric using hold-out method. The classifier was trained without Chaetoceros chains. Column and row heading are coded as: C1, copepod; C2, rod-shaped diatom chains; C3, Chaetoceros socialos colonies; C4, hydroid medusae; C5, marine snow; C6, Chaetoceros chains; C6*, "un-

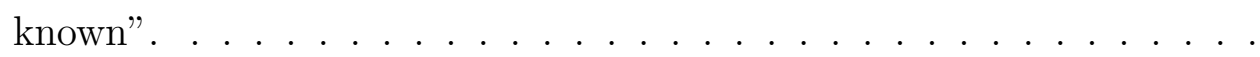


3.5 Confusion matrix of an LVQ-NN classifier with distance rejection metric using hold-out method. The classifier was trained without rodshaped diatom chains. Column and row heading are coded as: C1, copepod; C2, Chaetoceros chains; C3, Chaetoceros socialis colonies; C4, hydroid medusae; C5, marine snow; C6, rod-shaped diatom chains; C6*, "unknown". . . . . . . . . . . . . . . . .

3.6 Confusion matrix of an LVQ-NN classifier with distance rejection metric using hold-out method. The classifier was trained without copepod. Column and row heading are coded as: C1, rod-shaped diatom chains; C2, Chaetoceros chains; C3, Chaetoceros socialis colonies; C4, hydroid medusae; C5, marine snow; C6, copepod; C6*, "unknown". . . . . . .

4.1 Mean classification accuracy from different feature representation methods, where the unit is in percent. The abbreviations are as follows: MI - moment invariants, FD - Fourier descriptors, SS - shape spectrum, MM - morphological measurements, CM - co-occurrence matrices, RL run length, EF - edge frequency, PS - pattern spectrum, WT - wavelet transform. The best performance for single feature method is the cooccurrence matrices method, which has the average of classification accuracy of $74 \%$. It is clear to see that the texture-based methods are superior than shape-based methods. . . . . . . . . . . . .

4.2 Standard deviation of classification rates from different feature representation methods, where the unit is in percent. The abbreviations are same as Table $4.1 . \ldots \ldots \ldots \ldots \ldots$ 
5.1 Confusion matrix for EN302, VPR Tow 7, based on the co-occurence matrix classifier using hold-out method. Column and row heading are coded as: C1, copepod; C2, rod-shaped diatom chains; C3, Chaetoceros chains; C4, Chaetoceros socialis colonies; C5, hydroid medusae; C6, marine snow; C7, 'other'; and $P_{d}$, probability of detection. True counts (i.e. human counts) for a given taxa are given in the columns, while counts by automatic identification (i.e. computer counts) are given in the rows. Correct identifications by the computer are given along the main diagonal, while the off-diagonal entries are the incorrect identification by the computer. Overall accuracy for this classifier was $72 \% \ldots \ldots \ldots \ldots \ldots \ldots$

5.2 Mean confusion matrix for EN302, VPR Tow 7, based on learning vector quatization method neural network classifiers built with different randomly selected sets of 200 training ROIs using hold-out method [34]. Column and row headings are as in Table 5.1. True counts (i.e. human counts) for a given taxa are given in the columns, while counts by attomatic identification (i.e. computer counts) are given in the rows. The correct identifications by the computer are given along the main diagonal, while the off-diagonal entries are the incorrect identification by the computer. Overall accuracy of this classifier was $61 \%$. . . . .

5.3 Performance of the classifier with different kernel widths $(\sigma)$, regulation penalty (C) and kernel types, where $d$ is the polynomial degree and $\kappa$ is the kernel coefficient. The recognition rate on the independent test set is shown. . . . . . . . . . . . . . . . . . . .

5.4 Kullback-Leibler(KL) distance estimation for difference in abundance between COM-SVM and hand-sorted and between CSF-NN and handsorted. Row headings are as in Table 5.1. The KL distance is dimensionless. For two identical abundance curves, the KL distance is 0 , while for two random distributions, the KL distance is 0.5. Note lower values of COM-SVM than CSF-NN for all four taxa. . . . . . . . . 
6.1 Confusion matrix of the dual-classification system, using the leave-oneout method. Randomly selected images (200 per category) from EN302 VPR tow 7 were used to build the confusion matrix. C1: copepods, C2: rod-shaped diatom chains, C3: Chaetoceros chains, C4: Chaetoceros socialis colonies, C5: hydroid medusae, C6: marine snow, C7: other, $\mathrm{C} 7 *$ : unknown, $P_{D}$ : probability of detection $(\%), \mathrm{SP}=$ specificity (\%). NA: not applicable. True counts (i.e. human counts) for a given taxa are given in the columns, while counts by classification system are given in the rows. Correct identifications by the computer are given along the main diagonal, while the off-diagonal entries are the incorrect identification by the computer. All data are counts, except in the last row and last column, which are percent values. Although images from the "other" category are not needed to train the dual-classification system, they are necessary to evaluate it. . . . . . . . . . . . . 137

6.2 Confusion matrix of the single LVQ-NN classifier, using the leave-oneout method. Images used were the same as those in Table 6.1. Abbreviations as in Table 6.1. All data are counts, except in the last row and last column, which are percent values. . . . . . . . . . . . . . 140 


\section{Chapter 1}

\section{Introduction}

The vast majority of species in the ocean are plankton. The term plankton was coined by the German scientist Victor Henson at the University of Kiel in 1887 from the Greek word "planktos", meaning "drifter", to describe the passively drifting organisms in freshwater and marine ecosystems. Many species are planktonic for only part of their lives (meroplankton), including larvae of fish, crabs, starfish, mollusks, corals, etc. Other species are always planktonic (holoplankton), including the many species of phytoplankton and copepods. As primary producers, phytoplankton are responsible for approximately $40 \%$ of the annual photosynthetic production on earth. Phytoplankton and their predators, zooplankton, play important roles in processes such as the carbon cycle, the biological pump, global warming, harmful algal blooms and coastal eutrophication. As the base of the ocean food web, plankton play important roles in sustaining commercial marine fisheries. In order to better understand the marine ecosystem, knowledge of the size structure, abundance, mass, and species composition of plankton is crucial. Such measurements are difficult however, since plankton distributions are notoriously patchy and require high-resolution sampling tools for adequate quantification $[45,61,120,108]$. In spite of over a hundred years of research [168], our understanding of the structure of aggregations of plankton is still very limited. Taxa-specific abundance at both fine-scale temporal and spatial resolution is necessary to assess theoretical ecological models such as those of Riley [134], Fasham [46], Aksnes et al. [2], Lynch et al. [107], Miller et al. [115], and 
Carlotti et al. [17].

\subsection{Motivation}

The advent of new optical imaging sampling systems [31] in the last two decades offers an opportunity to resolve taxa-specifc plankton distribution at much higher spatial and temporal resolution than previously possible with net, pump, and bottle collections. Optical imaging systems rapidly create large amounts of digital image data and ancillary environmental data that need to be analyzed and interpreted. Analyzing the image data can be accomplished using manual processing by trained experts. In addition to the high cost of expert time, such classification processes are tedious and time-consuming, which can cause biased results [28]. On the other hand, advances in pattern recognition and machine learning make it possible to automatically classify plankton images into major taxonomic groups in real time. In this thesis, I take this approach and pursue the automatic classification of these images via statistical pattern recognition.

\subsection{Statistical pattern recognition}

Statistical pattern recognition has been used successfully in a number of applications such as data mining, document classification, biometric recognition, bioinformatics, remote sensing and speech recognition. In statistical pattern recognition, a pattern is represented by a set of measurements, called features. Each pattern then can be viewed as a point in the multi-dimensional feature space. Statistical learning theory is then applied to construct decision boundaries in the feature space to separate the different pattern classes. A recognition system is usually operated in two phases: training and classification, as shown in Figure 1-1.

Incoming video from an optical imaging system, in this case a Video Plankton Recorder (VPR) [31, 32, 33, 34, 35], is pre-processed by a focus detection program to extract in-focus objects, called regions of interest (ROI), from each video frame. These 
ROIs are saved as Tagged Image File Format (TIFF) image files. A subset of these files is manually labeled (identified), and serves as training samples. In the training phase, a set of measurements (features) is computed from each image using different pattern representation methods. Feature extraction is used to linearly combine different features and extract the most salient features for classification. Subsequently, to train a classifier, a learning algorithm is employed to partition the feature space into subspaces belonging to different classes (e.g., species). An important feedback path allows a designer to interact with and optimize different pattern representation methods, feature extraction algorithms and learning strategies. The arrows of pattern representation and feature extraction between training and classification phases imply that the same methods are used in classification which are optimized during training. In the classification phase, the trained classifier uses the image-to-feature mapping, which is learned during training, and assigns an input image to a class based on its location relative to decision boundaries in the feature space.

\subsubsection{Features}

Features are measurable heuristic properties of patterns of interest. The rationale of pattern representation and feature extraction is to avoid the curse of dimensionality [8], the exponential growth of hypervolume as a function of dimensionality. For most practical systems, labeled samples require expert time, thus are expensive to obtain, that is to say, only limited labeled samples are available. In such cases, it has been observed that additional features may degrade the classifier performance, which is referred to as the peaking phenomenon [76, 130, 129]. Thus a dimensionality reduction (feature extraction and selection) step is essential, where only a small number of the most salient features are selected to improve the generalization performance (classification performance on samples "unseen" during training) of a classification system. At the same time, this step also reduces the storage requirements and processing time. 


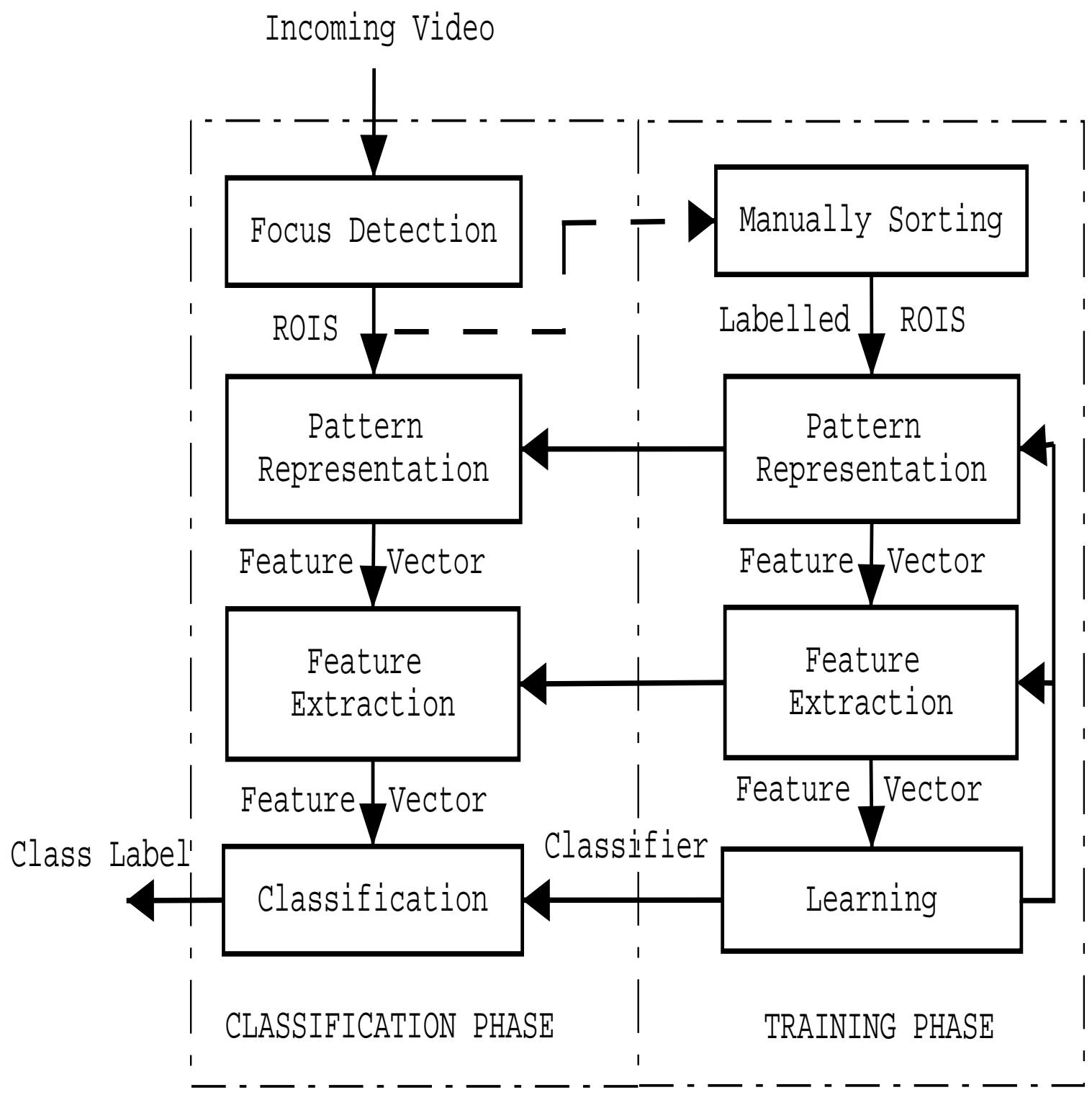

Figure 1-1: Schematic diagram of the pattern recognition system. 


\subsubsection{Statistical learning theory}

The fundamental work of Vapnik $[159,160,161]$ set the foundation for learning from finite samples by using a functional analysis perspective with modern advances of probability and statistics, and revived classical regularization theory. The basic idea of Vapnik's theory is to limit the model capacity by constraining decision boundaries in a "small" hypothesis space, which is dependent on the training samples. This is closely related to classical regularization theory in machine learning and overfitting/overtraining in pattern recognition.

More formally, learning from examples can be formulated in the statistical learning theory framework. Suppose we have two sets of variables $\mathbf{x} \in \mathbf{X} \subseteq R^{d}$ and $y \in Y \subseteq R$. A probability density function $p(\mathbf{x}, y)$ relates these two sets of variables over the whole domain $\mathbf{X} \times Y$. We are provided with a data set $D_{l} \equiv\{(\mathbf{x}, y) \in \mathbf{X} \times Y\}^{l}$. They are called the training data, and are obtained by sampling the probability density function $p(\mathbf{x}, y) l$ times. Given the data set $D_{l}$, the problem of learning lies in providing an estimator (a classifier/a learning machine) as a function $f_{\alpha}: \mathbf{X} \rightarrow Y$, which can be used to predict a value of $y_{i}$ given any value of $\mathbf{x}_{i} \in \mathbf{X}$. The functions $f_{\alpha}(\mathbf{x})$ are different mappings with adjustable parameters $\alpha$. A standard way to solve the above learning problem is to define a risk function, which computes the average amount of error (cost) associated with an estimator, then choose the estimator which has the lowest risk. The expected risk of an estimator is defined as,

$$
R\left(f_{\alpha}\right)=\int_{\mathbf{x}} \int_{y} V\left(f_{\alpha}(\mathbf{x}), y\right) p(\mathbf{x}, y) d \mathbf{x} d y
$$

Here $V$ is the loss function, and $\alpha$ are adjustable parameters. A particular choice of $\alpha$ determines a learning machine. For example, a neural network with fixed architecture is a learning machine, where $\alpha$ are the weights and bias of the network. The target estimator is the function $f_{\alpha^{*}}$ which has minimal expected risk,

$$
f_{\alpha^{*}}(\mathbf{x})=\arg \min _{\alpha} R\left(f_{\alpha}\right)
$$


In practice, the probability density function $p(\mathbf{x}, y)$ is unknown, and the expected risk cannot be calculated using Eq. 1.1. To overcome this problem, an induction principle is used to approximate the expected risk from training samples. This is the so-called empirical risk minimization (ERM) induction approach. The empirical risk is defined as,

$$
R_{e m p}\left[f_{\alpha}\right]=\frac{1}{l} \sum_{i=1}^{l} V\left(y_{i}, f_{\alpha}\left(\mathbf{x}_{i}\right)\right) .
$$

For limited training samples, the empirical risk is not always a good indicator of the generalization ability of a learning machine. The structural risk minimization principal [160] states that, for any $\alpha \in \Lambda$ and $l>h$, the following bound holds with a probability of of at least $1-\eta$,

$$
R(\alpha) \leq R_{e m p}(\alpha)+\sqrt{\frac{h\left(\log \frac{2 l}{h}+1\right)-\log \left(\frac{\eta}{4}\right)}{l}} .
$$

The parameter $h$ is a non-negative integer called the Vapnik Chervonenkis (VC) dimension. It is a measurement of capacity of a set of functions. The second term on the right side of Eq. 1.4 is called the $V C$ confidence. Consequently, the essential idea of structural risk minimization can be restated thus: for a fixed sufficiently small $\eta$,

choose the function $f_{\alpha}(\mathbf{x})$ which minimizes the right hand side of Eq. 1.4. For more information on this topic, please refer to Vapnik [160, 161], Burges [15], and Evgeniou [44].

\subsection{An overview of related work}

Research on automatic plankton classification has been on-going for many years [82, 81, 135, 69, 25]. Early systems worked on images taken under well-controlled laboratory conditions, and had not been applied to field-collected images. More recently, artificial neural networks have come to play a central role in classifying plankton images $[145,12,27,150,149,154,28]$. However, the datasets used to develop and test these classifiers were usually fairly small $[150,28]$, and, furthermore, only a subset of 
distinctive images was chosen to both train and test the classifier. Since a classifier needs to classify all the images from the field, including rare species and difficult ones, even those that cannot be identified by a human expert, the accuracy reported for a classifier built from only distinctive images will be generally optimistically biased. The classifier performance was usually much worse when it was applied to all field data [34].

The features used in the early systems were mostly shape-based. Jeffries et al. [81] used moment invariants, Fourier descriptors and morphometric relations as features. Although these features worked quite well under well-defined laboratory imaging conditions and the overall recognition rate reported by Jeffries et al. was $90 \%$ for six taxonomic groups, the system required significant human interaction and was not suitable for in situ applications.

Initial automatic identification of VPR images was carried out using the method described in Tang et al. [150] which introduced granulometry curves [162], along with traditional features such as moment invariants, Fourier descriptors and morphometric measurements. This method used a learning vector quantization (LVQ) neural network as the classifier [149] and achieved $92 \%$ classification accuracy on a subset of VPR images for six taxonomic groups. Only distinctive images were used in training and testing the classifier in this initial study. A detailed experiment was conducted in Chapter 3 to show the performance of the system when rare species and difficult images were included in training or testing samples. The average classification performance on the whole dataset was $61 \%$ [34].

The performance disagreement between previous methods [81, 150] and current study [34] is due to the nature of field-captured images. Unlike the well-controlled laboratory conditions, field images are often occluded (objects truncated at edge of image), and shape-based features such as moment invariants and Fourier descriptors are very sensitive to occlusion. In addition, a significant number of field-collected images cannot be identified by a human expert due to object orientation and position in the image volume ${ }^{1}$. These unidentifiable images were not used in training and testing

\footnotetext{
${ }^{1}$ Objects can be hard to identify due to their position in the image volume. If part of the object
} 
the classifier [150] (although occluded images were included). A recent study by Luo

et al. [106] showed that including unidentifiable objects lowered the recognition rate from $90 \%$ to $75 \%$ for their dataset from the shadow image particle profiling evaluation recorder. In order to better estimate species specific abundance, a number of works has shown that it was important to include an "other" [34] or "reject" [58] category.

In addition to occlusion, nonlinear illumination of images makes perfect segmentation (binarization) impossible, even after background brightness gradient correction. Due to the grayscale gradient, the same object can have different segmented shapes depending on where the object is in the field-of-view, thus causing shape-based features to be less reliable.

Another type of feature we can extract from the grayscale images is a texture-based feature. However, due to the early success of shape-based features on plankton images from well-controlled laboratory imaging conditions, texture-based features have not been widely used in plankton image recognition.

Texture-based features were compared against classic shape-based features. The important finding was that the texture-based features were more important than the shape-based features to classify field-collected plankton images. The main cause was that texture-based features were less sensitive to occlusion and projection variance than shape-based features.

\subsection{Data}

The data set was obtained from a 24-h VPR tow (VPR-7) in the Great South Channel off Cape Cod, Massachusetts, during June 1997 on the R/V Endeavor. The VPR was towed from the ship in an undulating mode, forming a tow-yo pattern between the surface to near bottom. The images were taken by the high magnification camera, which had an image volume of $0.5 \mathrm{ml}$. The total sampled volume during the

is out of this volume, the resulting image will be occluded. Nonlinear illumination makes objects from the dark region more likely to be occluded by global segmentation, a problem correctable by background gradient removal [35] 
deployment was approximately $2.6 \mathrm{~m}^{3}{ }^{2}$. There were over 20,000 images captured during this tow. All the images were manually identified (labeled) by a human expert into seven major categories (copepod, rod-shaped diatom chains, Chaetoceros chains, Chaetoceros socialis, hydroid medusae, marine snow, and the "other" category, comprising rare taxa and unidentifiable objects). These are the most abundant categories in this area. In this tow, about $21 \%$ of the images belonged to the "other" category. Most of these "other" images were unidentifiable by human experts, and the rest were rare species, including coil-shaped diatom chains, ctenophores, chaetognaths, polychaetes and copepod nauplii (see Davis et al. [34]). The manual identification took several weeks to accomplish. Representative samples (images) are shown in Figs. 1-2, 1-3, and 1-4. Manual labels were treated as ground truth for comparing different classification results.

\subsection{Thesis overview}

This thesis consists of seven chapters and is organized as follows.

Chapter 1: Introduction- I introduce the importance of automatic classification of plankton images. I then set up the problem in the framework of statistical pattern recognition, and review basic concepts on statistical learning and related work. Finally, I describe the data set used in this thesis.

Chapter 2: Data acquisition- I give an overview of water column plankton samplers, and then focus on the Video Plankton Recorder (VPR). I develop three algorithms of focus detection and examine four short sections of video. I then compare the results from three algorithms to the manual examination in terms of probability of detection and probability of false alarm.

Chapter 3: Classification method: analysis and assessment- I present a detailed assessment of the application of a learning vector quantization neural network (LVQ-NN) on the data set. More specifically, I examine the following: classifier

\footnotetext{
${ }^{2}$ As pointed out in Davis et al. [35], although the volume imaged by VPR is small compared to the volume filtered by a plankton net, the VPR still can provide an equivalent or better estimate of plankton abundance.
} 
Copepods

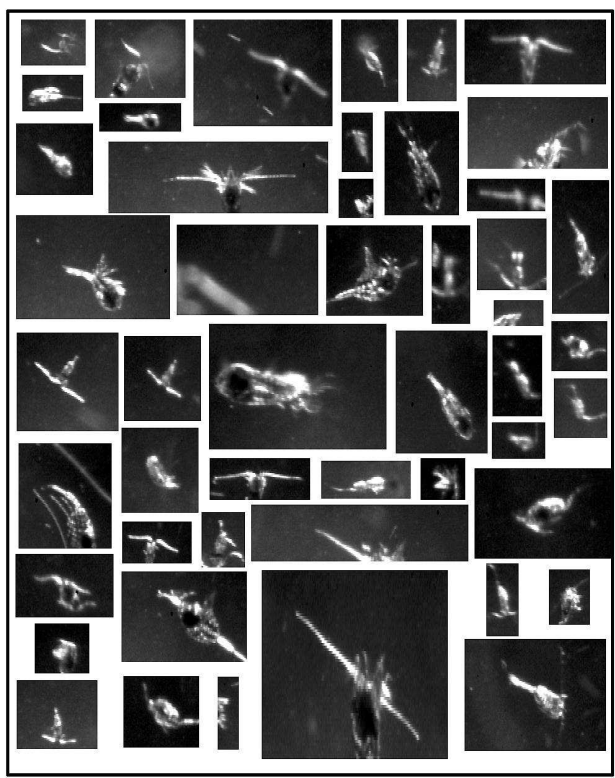

Chaetoceros socialis colonies

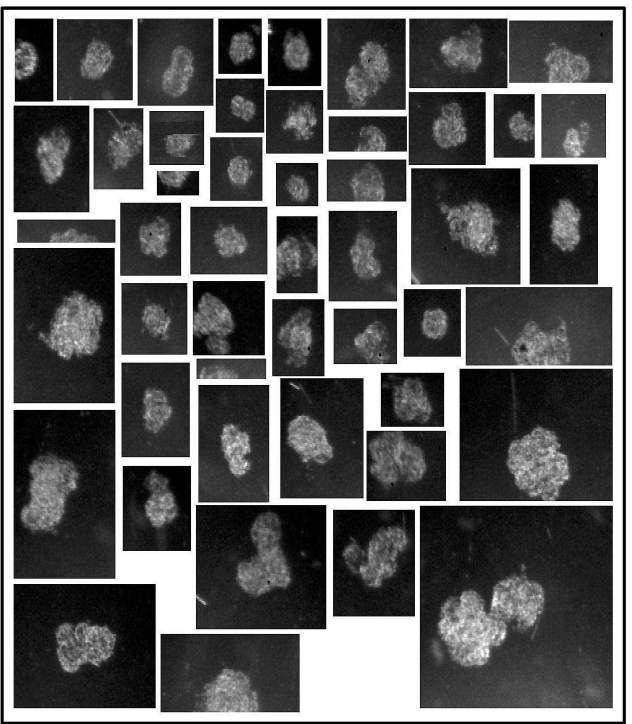

$1 \mathrm{~mm}$

Rod-shaped Diatom Chains

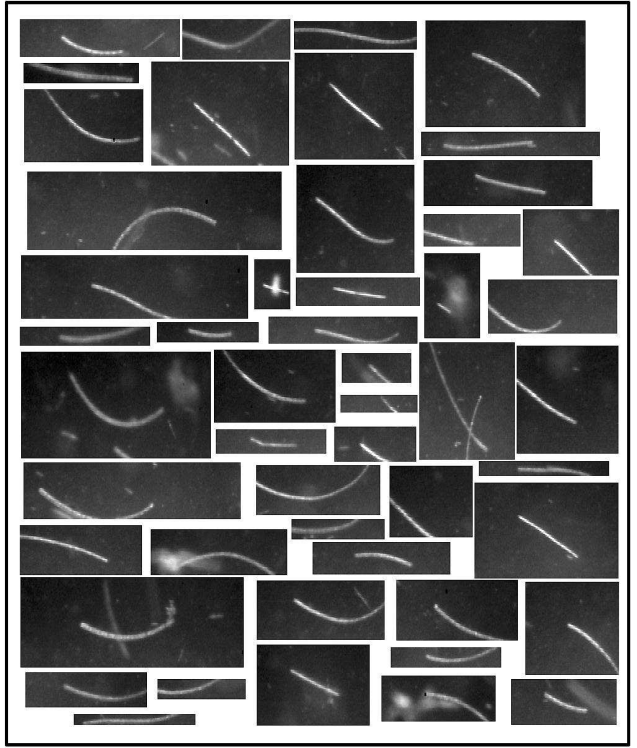

Other

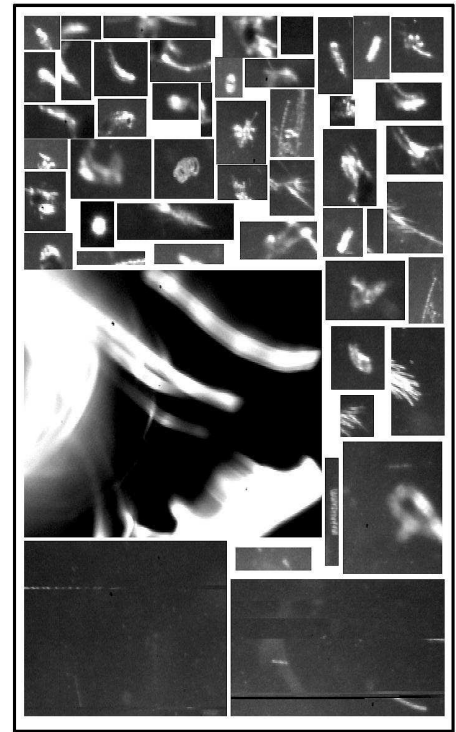

Figure 1-2: Example VPR images of copepods, rod-shaped diatom chain, Chaetoceros socialis colonies and the "other" category. Fifty randomly selected samples are shown here. 
Chaetoceros chains

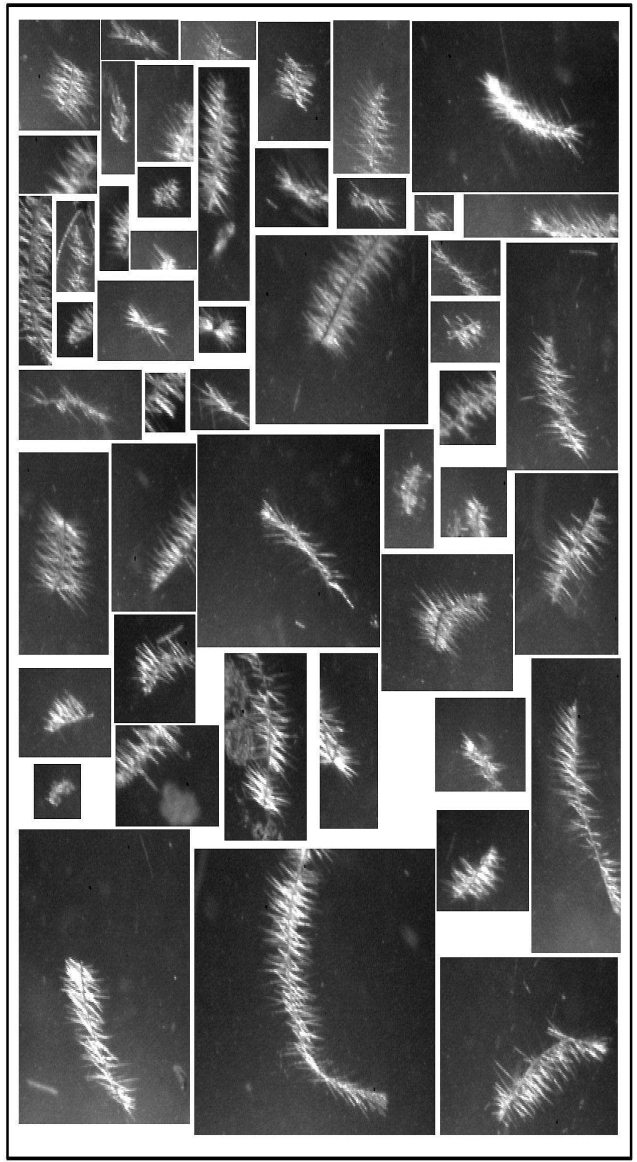

Marine Snow

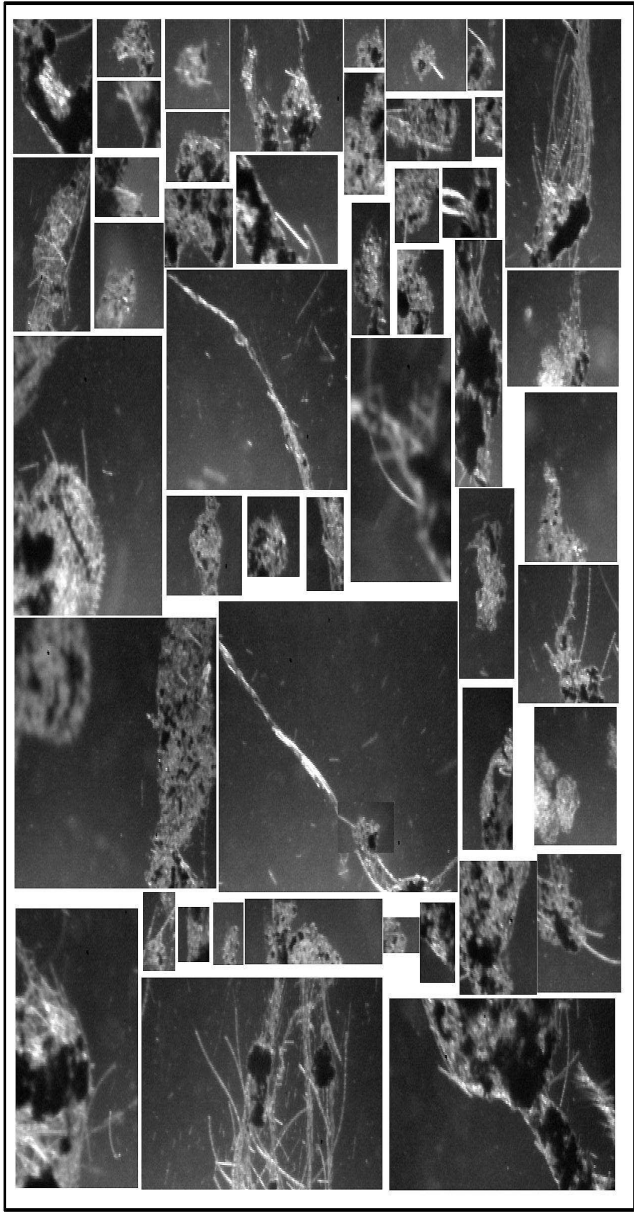

$-1 \mathrm{~mm}$

Figure 1-3: Example VPR images of Chaetoceros chains and marine snow. Fifty randomly selected samples are shown here. 
Hydroid Medusae

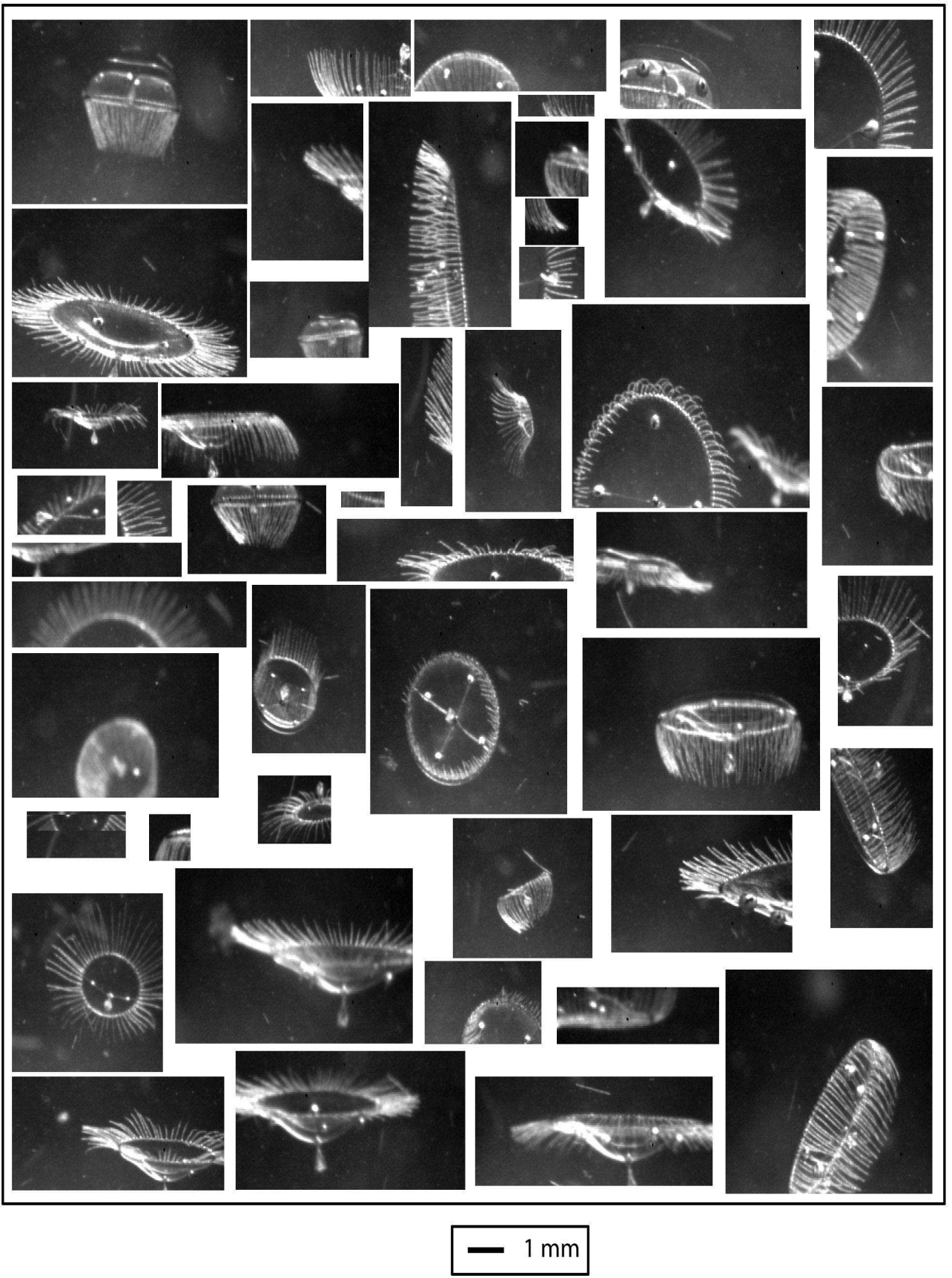

Figure 1-4: Example VPR images of hydroid medusae. Fifty randomly selected samples are shown here. 
complexity, feature length, learning curve, presentation order of training samples, and different training samples. Next I propose a two-pass classification system and compare the result with both the single LVQ-NN classifier and the single LVQ-NN classifier with statistical correction. Finally, I modify the LVQ-NN to have an outlier rejection metric based on the mean distance of correctly classified training samples.

Chapter 4: Pattern presentation- First I give an overview of pattern representation/feature measurement methods. I group the pattern presentation methods into three major groups, namely, shape-based, texture-based, and other methods. I then conduct a comparison study between shape-based features and texture-based features on a random set of the plankton data. I find the texture-based features are more important than shape-based features to classify field-collected images. I keep the comparison results as guidelines for choosing different feature presentation methods in the later chapters.

Chapter 5: Co-occurrence matrices and support vector machine- I investigate the multi-scale co-occurrence matrices, and support vector machines to classify the plankton image data set. From Chapter 4, I find that texture-based features are more robust for classifying field-collected plankton images with occlusions, nonlinear illumination and projection variance. I demonstrate that by using features from multi-scale co-occurrence matrices and soft margin Gaussian kernel support vector machine classifiers, a $72 \%$ overall probability of detection can be achieved compared to that of $61 \%$ from a neural network classifier built on combinded shape-based features. Subsequent plankton abundance estimates are improved in regions of low relative abundance by more than $50 \%$.

Chapter 6: Dual classification system- I incorporate a learning vector quantization neural network classifier built from combined shape-based features and a support vector machine classifier with texture-based features into a dual-classification system. The system greatly reduces the false alarm rate of the classification, thus extends the regions where the specificity curve of classification is relative flat, which makes global correction of abundance estimation possible. After automatic correction, the abundance estimation agrees very well both in high and low relative abundance 
regions. For the first time, I demonstrate an automatic method which achieves abundance estimation as accurately as human experts.

Chapter 7: Conclusions and future work- First, I summarize the major contributions of this thesis, and then discuss the possibility of extending the existing system to color or 3-D holographic images. 


\section{Chapter 2}

\section{Data Acquisition}

In this chapter, I first overview water column plankton samplers in Section 2.1, then decribe one specific optical sampler, the Video Plankton Recorder, in detail in Section 2.2. The main focus of this chapter is to discuss the focus detection program, which is discussed in Section 2.3. I develop three new focus detection algorithms, and compare them against human judgment on four video sections from VPR. This is the first quantitative study of focus detection.

\subsection{Water column plankton samplers}

The development of quantitative zooplankton sampling systems can be traced back to the late 19th and early 20th centuries. Non-opening/closing nets [67, 83], simple opening/closing nets [71] and high-speed samplers [4] all began to be employed at that time. All these systems have evolved with advances in technology, and are still widely used for plankton survey programs. For example, non-opening/closing nets, such as the Working Party 2 (WP2) net [49], modified Juday net [1], and Marine Resources Monitoring Assessment Prediction (MARMAP) Bongo net [126] are still used in large ocean surveys; simple opening/closing nets similar to those developed by Hoyle [71], Leavitt [96], Clarke and Bumpus [24] are still manufactured and used; high-speed samplers are also in use, such as the continuous plankton recorder [60], which has evolved over 30 years, and become the main sampling system in the North 
Atlantic plankton survey [164].

Since the 1950s, the concept of plankton patchiness has been well established, and it triggered the development of closing cod-end systems and multiple net systems in the 1950s and 1960s. Cod-end samplers such as the Longhurst-Hardy plankton recorder [103] had problems with hang-ups and stalling of animals in the net which caused smearing of the distributions of animals and loss of animals from the recorder box [63]. The system was modified by Haury et al. to reduce these sources of bias and used to study plankton patchiness in a variety of locations [62,64]. Multiple net systems $[169,172]$ were developed to fix these problems by opening and closing nets in specific portions of the water column.

With the advances in charge-coupled device (CCD) and computer technology, the 1980s and 1990s saw a boom of optical plankton sampling systems. Optical systems have a number of advantages over net-based systems. The optical systems can provide much finer vertical and horizontal spatial resolution than the net-based systems. Optical systems have the potential to provide abundance estimates at short temporal intervals along the tow path [32]. Furthermore, delicate and particulate matter that may be damaged by net collection can be quantified by optical systems $[5,38]$. Image-forming systems have the potential to map taxa-specific distribution in real time [34]. However, optical systems usually have a smaller sampling volume than net-based systems given the same tow length. Thus rare organisms may remain undetected with optical sampling systems.

Optical systems can be divided into two categories depending on whether the system produces images of organisms or not. Non-image-forming systems such as the optical plankton counter [68] use the interruption of a light source to detect and estimate particle size. The family of image-forming systems has grown continuously since 1990. The Ichthyoplankton Recorder (IR) [50, 99], Video Plankton Recorder (VPR) [31], Underwater Video Profiler (UVP) [55], Optical-Acoustic Submersible Imaging System (OASIS) [75], In situ Video Camera [152], FlowCam [144], Holocamera [88], Shadowed Image Particle Profiling and Evaluation Recorder (SIPPER) [138], Zooplankton Visualization and Imaging System (ZOOVIS) [10], HOLOCAM [166], In 
situ CritterCam [147], and Optical Serial Section Tomography (OSST) [48] all belong to this category. In this thesis, images from the VPR were used. However, the algorithms developed in this thesis are generic, and readily applied to images from other optical plankton sampling systems.

Another group of plankton sampling systems is acoustic-based [170, 47]. Such systems use acoustic backscattering to measure the size distribution of particles and plankton. Hybrid systems also have been developed, combining optical and acoustic sampling, e.g., the VPR has been combined with multifrequency acoustics on the BIo-Optical Multi-frequency Acoustical and Physical Environmental Recorder (BIOMAPER-II) [173]. For more detailed review of plankton sampling systems, please refer to Wiebe and Benfield [168].

Imaging plankton at sea while towing the sampler through the water at a $1-6 \mathrm{~m} / \mathrm{s}$, requires a combination of magnifying optics, short exposure time, and long working distance $(0.5 \mathrm{~m})$. The long working distance is needed to minimize detection and avoidance of the sampler by the plankton. The short exposure time (e.g., $1 \mu \mathrm{s})$ is obtained using a strobe. The density of pixels on the CCD array, together with the need to image enough details of the individual plankton to identify them, limits the camera's field-of-view (FOV) to $1 \mathrm{~cm}$ for most mesozooplankton. For a depth of focus of $3 \mathrm{~cm}$, the image volume is $3 \mathrm{~cm}^{3}$, and video rate of 60 fields per second (FPS), yields 0.18 liter of water imaged per second. Given a typical coastal concentration of mesozooplankton of 10 individuals per liter, the time between individual sightings is 0.55 seconds, and at $60 \mathrm{FPS}$, there are 33 video fields between sightings. Thus, only a small fraction of the video fields will contain mesozooplankton. For typical survey periods of several hours or days, the volume of video data collected is much too large for human operators to process manually. (For example, VPR has the bandwidth of $6 \mathrm{Mb} / \mathrm{s}$ or $518 \mathrm{~Gb} /$ day). Automatic pre-processing of the data is essential [31, 33]. In this chapter, I focus on one such pre-processing method called focus detection. Before discussing this method, a detailed description of the VPR is necessary. 


\subsection{Video Plankton Recorder}

The VPR system includes an underwater unit with video and environmental sensors, and a deck unit for data logging, analysis and display (Figure 2-1). The underwater unit has a video system with magnifying optics that images plankton and seston in the size range of 100 microns to several centimeters [31, 33, 34, 35]. The initial design [31] had four SONY XC-77 CCD cameras configured to simultaneously image concentric volumes at different magnifications. The fields of view of the four cameras were $0.7 \times 0.56,3 \times 2.4,5 \times 4$, and $8 \times 6.4 \mathrm{~cm}^{2}$ respectively. Depths of field were adjustable by different aperture settings. The sampled image volumes in each field ranged from $0.5 \mathrm{ml}$ to 1 liter depending on the optical settings. The modified system $[33,34]$ had two analog video cameras of high and low magnification respectively. The high magnification camera had an image volume of about $0.5 \mathrm{ml}$ per field, while the low magnification camera had an image volume of about $33 \mathrm{ml}$ per field. Early testing determined that these two cameras provided the most useful information. The high-magnification camera provided detailed images permitting identification to the species level, while the low-magnification camera imaged larger organisms such as ctenophores and euphausiids. Positioning the image volume at the leading edge of the tow-body and having a wide separation of the cameras and strobe, permitted imaging of animals in their natural undisturbed state.

The images studied in this thesis came from the high magnification camera, which had a pixel resolution of about 10 microns. The cameras were synchronized at 60 fields per second to a xenon strobe ${ }^{1}$. The VPR also included a suite of auxiliary sensors that measured pressure, temperature, salinity, fluorescence, beam attenuation, downwelling light, pitch, roll, velocity and altitude. The environmental and flight control sensors were sampled at 3 to $6 \mathrm{~Hz}$. The underwater unit was towyoed at $4 \mathrm{~ms}^{-1}$ using a $1.73 \mathrm{~cm}$ diameter triple-armor electro-optical cable. Video and environmental data from the towbody were received via a fiber optic cable into the data logging and

\footnotetext{
${ }^{1}$ The current system has a single $1008 \times 1018$ digital camera with field of view from $5 \times 5 \mathrm{~mm}^{2}$ to $20 \times 20 \mathrm{~mm}^{2}$, and the depth-of-field is objectively calibrated using a tethered organism. The images were sampled at 30 frames per second [35]
} 


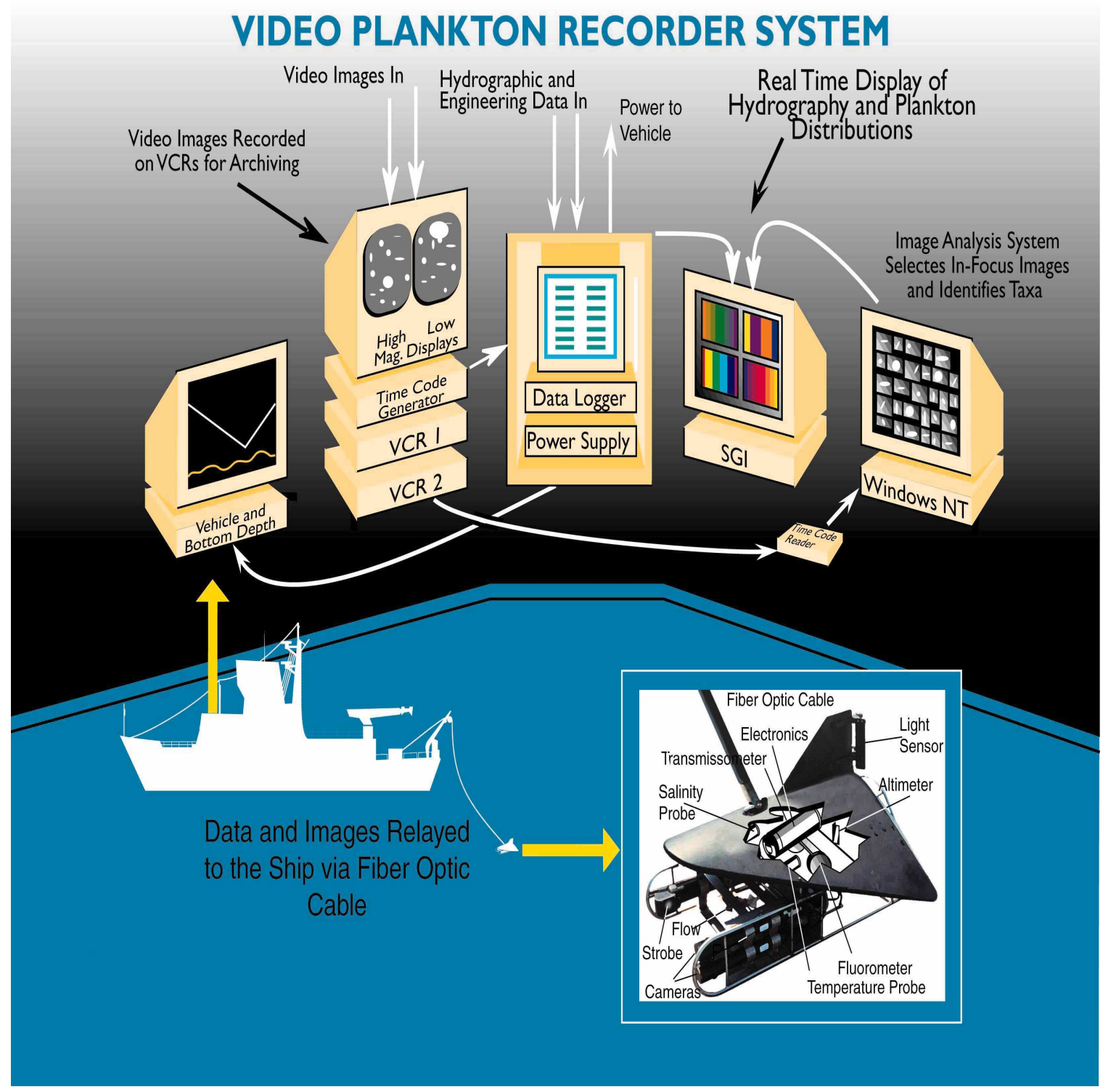

Figure 2-1: Video Plankton Recorder system with underwater and shipboard components. The VPR is towyoed at ship speeds up to $5 \mathrm{~m} / \mathrm{s}$, while video is processed in real-time on board. 
focus detection computer on the ship.

The deck unit consisted of a video recording/display system, an environmental/navigational data logging system, an image processing system and a data display system. Video was time-stamped at 60 fields per second and recorded on SVHS recorders. The video time code was synchronized with the time from the P-code Global Positioning System. Latitude and longitude were logged with video time code and environmental data at $3 \mathrm{~Hz}$ on a personal computer and a Silicon Graphics Inc (SGI) workstation.

\subsection{Focus Detection}

Video with time code from the high magnification camera was sent to the focus detection system, which included an image processor interfaced to a computer. Video was first digitized at field rates, then in-focus objects were detected using an edge detection algorithm. The regions of interest (ROI) were saved to the hard disk as tagged image format files using the video time code as the filename.

\subsubsection{Objective}

The main objective of the focus detection algorithm is data reduction. The video comes in from the video camera at 60 fields per second. As discussed above, a large proportion of fields are devoid of in-focus objects. Early systems required a human operator to scan through all the video fields to determine when an in-focus organism was observed and to what species it belonged. Such processes were very slow and tedious, and introduced a source of subjective error when a line was drawn between in-focus and out-of-focus objects. This line could vary from person to person, and from time to time. The objective of the focus detection algorithm is to replace the human operator with a program which objectively extracts in-focus objects from the video images. The focus detection algorithm is required to extract as many in-focus objects as possible, while picking up as few out-of-focus objects as possible, all in real time. More formally, the focus detection program needs to have a high probability 
of detection, while maintaining a low probability of false alarm. A graphic user interface (GUI) is available to select parameters such as segmentation threshold, Sobel threshold, growth scale, minimum blob size, and minimum join distance (Figure 2-2). Choosing different parameters sets the tradeoff between the probability of detection and the probability of false alarm. A high probability of detection usually related with a high probability of false alarm, which increased the level of difficulty of the subsequent classification problem and required more disk space. On the other hand, low probability of false alarm was related with a low probability of detection. The effective sampling volume was reduced. A compromise between the probabilities of detection and false alarm needed to be made by adjusting the controlling parameters in the focus detection GUI.

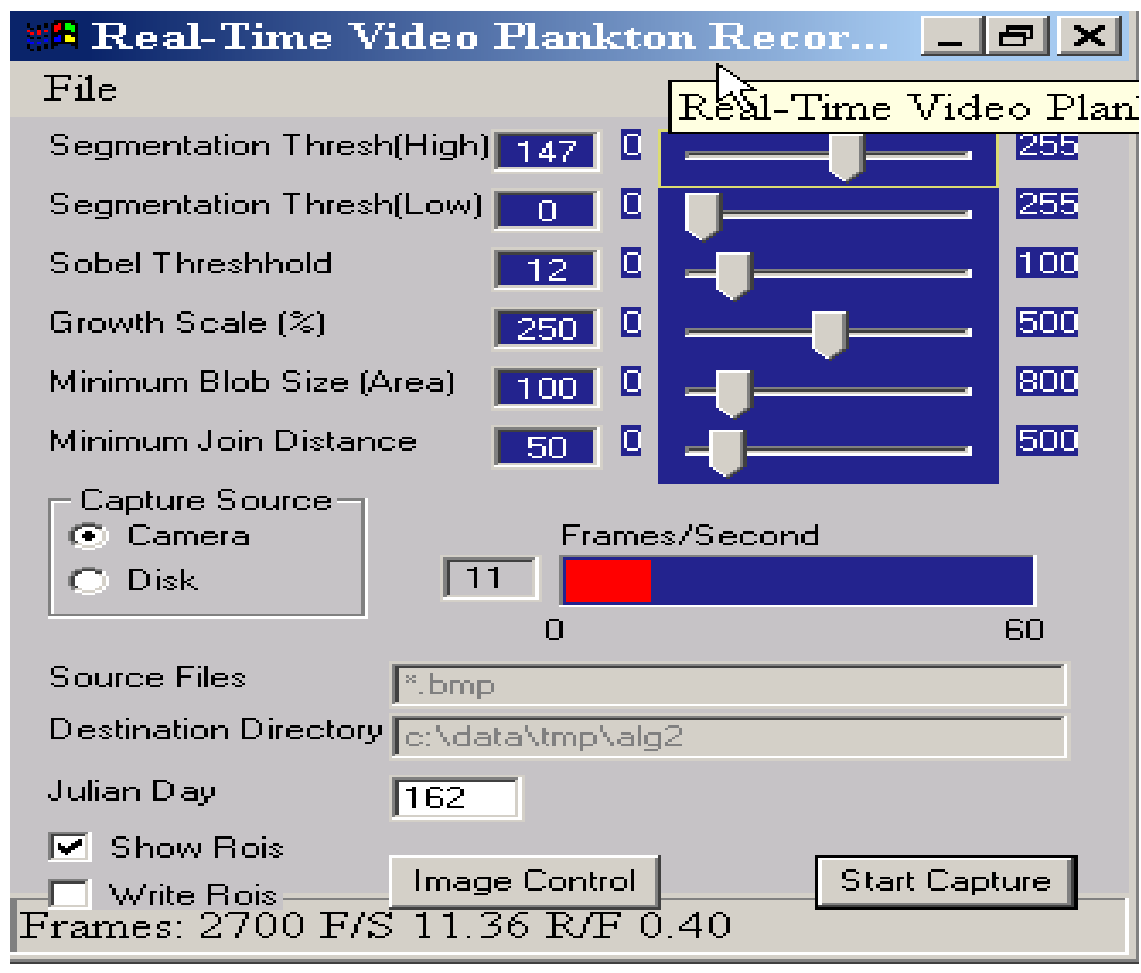

Figure 2-2: The graphical user interface of real time focus detection program. 


\subsubsection{Method}

In-focus object detection involves brightness correction, segmentation, labeling, size thresholding, edge detection, edge thresholding, coalescing and ROI generation. Incoming videos are dynamically adjusted to correct temporal changes in mean brightness by shifting the mean brightness of each video frame to a certain value. Translation instead of scaling is used in this normalization step to avoid changing brightness gradients within the frame. Brightness correction is followed by segmentation which involves binarization of gray-scale images into binary images. Pixels with brightness above the threshold value are set as foreground while the rest of the pixels are set as background. After segmentation, a connectivity algorithm is used to check how the foreground pixels connect to form blobs. The distinct blobs then are labeled from 1 to $N$, where $N$ is the number of blobs present in the video field. Due to the imaging environment, there are many small blobs present in each frame. Since small objects are impossible to identify in the later processing and require much processing time, a size threshold is imposed, and consequently blobs below a minimum number of pixels are ignored. A rectangular bounding box is placed around each blob which passes size thresholding. A Sobel operator is applied to each blob to calculate the brightness gradient of the subimages. The small gradients in the subimages are considered to be noise instead of real edges, and the gradients of each subimage are further thresholded in order to suppress this noise.

Three in-focus algorithms are developed based on these thresholded gradients. If the blob is in-focus, the center position and size are saved. After in-focus checking on all the blobs from one field is completed, the bounding box of an in-focus blob is extended/shrunk according to the GUI growth scale setting. Planktonic organisms usually are partially transparent or translucent. When binarized, one organism often breaks into several blobs. A coalesce operation is applied to group the close in-focus blobs into one blob. Two or more blobs are considered to coalesce if there are overlaps after the bounding boxes relax or if the central distance between them is below a userdefined value on GUI. The resulting subimage inside the bounding box is called region 
of interest (ROI), and is written to the disk as Tagged Image File Format with ROI capture time as filename.

\subsubsection{Algorithms}

The motivation of the following algorithms is based on the observation that sharp in-focus objects usually have strong edges (high gradient) between themselves and their background, as well as inside themselves; while out-of-focus objects usually lack such features. However, there are always exceptions. One such exception is that highly saturated objects often reveal strong gradient between the objects and their background whether the objects are in-focus or not. Such artifacts are due to saturation of the objects. Three heuristic algorithms were developed to decide whether an object was in-focus based on the gradient information.

1. Algorithm A1 (edge pixels only):

A1 is an algorithm which ignores the strength of the gradient after the pixel is determined as edge pixel. The number of edge pixels is defined as the number of pixels whose gradient values are greater than some user specified threshold. The focus level index is defined as,

$$
F_{L}=\frac{N_{e}}{A}
$$

where $F_{L}$ is the focus level index, $N_{e}$ is the number of edge pixels, and $A$ is the area which is the number of foreground pixels in the subimage. The object is considered in-focus if $F_{L}$ is greater than a fixed value.

2. Algorithm A2 (edge strength and additive brightness correction):

A2 is an algorithm which makes use of the number of edge pixels and their gradient strength. In order to eliminate over-saturated blobs, which appear to have a strong gradient at the boundary, a brightness compensation is made to penalize such instances. The additive brightness correction is used in this approach. The additive brightness correction is calculated as the difference 
between the mean brightness in the subimage and the mean brightness of the field. The focus level index is calculated as,

$$
F_{L}=\frac{A}{4 \times N_{e}} \times \sum_{i=1}^{N_{e}} G_{i}-B_{c}
$$

$G_{i}$ is the gradient values from the subimage above a certain threshold, $A$ is the area of subimage defined as in A1, $N_{e}$ is the number of edge pixels whose gradient values are above a certain threshold, and $B_{c}$ is the additive brightness correction term. An object is considered to be in-focus when $F_{L}$ is greater than a user specified threshold.

3. Algorithm A3 (edge strength and multiplicative brightness correction): A3 is an algorithm which uses only the gradient strength of edge pixels as well as a multiplicative brightness correction. The multiplicative brightness correction is calculated as the differences between the brightness in the subimage and the mean brightness of the field. The focus level index was calculated as follows,

$$
F_{L}=c \times \frac{\sum_{i=1}^{N_{e}} G_{i}}{\sum_{j=1}^{N_{s}} B_{c}},
$$

where $F_{L}$ is focus level index, $c$ is a scaling constant, $N_{e}$ is the number of edge pixels defined as in $\mathrm{A} 2, G_{i}$ is the gradient values from each subimage, and $N_{s}$ is the number of pixels in the subimage. $B_{c}$ is the multiplicative brightness correction term.

\subsubsection{Result}

Two video sections of the high magnification camera from cruise AN9703 in Massachusetts Bay conducted during March 11-15 1997 were manually examined and used to "ground truth" the results of the three algorithms described above. The videos were originally recorded on SVHS tape and later dubbed to BETACAM-SP tape. The rationale of using BETACAM tape was to allow the human operator to go 
through the videos field by field more easily. During the manual counting process, a human operator examined each field with the assistance of the segmentation program. The total number of all the objects (numbers of blobs in segmented image) as well as the number of in-focus objects in each field were recorded. Extremely high concentrations of the colonial planktonic alga Phaeocystis were observed on the examined tape. Only two seconds of video were examined, for each of two sections. Three focus detection algorithms were tested on these two sections of video. The outputs of each algorithm were further examined by the same human operator, and the number of in-focus/out-of-focus images was counted. The results are summarized in Tables 2.1, and 2.2 .

Table 2.1: Comparison of focus detection algorithms from AN9703, high magnification camera, video section 1 . The numbers are blob counts; probability of detection $P_{d}$ and probability of false alarm $P_{f}$ are given as percentages.

\begin{tabular}{||l|l|l|l|r||}
\hline \hline Methods & In-focus & Out-of-focus & $P_{d}$ & $P_{f}$ \\
\hline Manual count & 132 & 808 & NA & NA \\
\hline A1 & 70 & 10 & $53 \%$ & $1.2 \%$ \\
\hline A2 & 75 & 11 & $57 \%$ & $1.4 \%$ \\
\hline A3 & 77 & 13 & $58 \%$ & $1.6 \%$ \\
\hline \hline
\end{tabular}

Table 2.2: Comparison of focus detection algorithms from AN9703, high magnification camera, video section 2 . The numbers are blob counts; probability of detection $P_{d}$ and probability of false alarm $P_{f}$ are given as percentages.

\begin{tabular}{||l|l|l|l|r||}
\hline \hline Methods & In-focus & Out-of-focus & $P_{d}$ & $P_{f}$ \\
\hline Manual count & 169 & 698 & NA & NA \\
\hline A1 & 82 & 8 & $49 \%$ & $1.1 \%$ \\
\hline A2 & 89 & 15 & $46 \%$ & $2.1 \%$ \\
\hline A3 & 87 & 11 & $51 \%$ & $1.6 \%$ \\
\hline \hline
\end{tabular}

The relative low probability of detection was due to the bottle-neck of the ROI file-writing process, since there was an extremely high rate of ROI detection for Phaeocystis. The whole process was synchronized in real time. Each field had only 16 milliseconds of processing time at most (since the video rate was $60 \mathrm{FPS}$ ). If it took too long to process one field, the following fields would be skipped. In order 
to take this bottleneck into account, the focus detection algorithms were run on a paused field which had one in-focus object (but still output the video signal at 60 FPS). The number of files which were written out during a one-minute interval was counted. The ratio between this number and the ideal number (3600 in this case) was the correction factor due to the slow-down caused by the disk writing process. The $P_{d}$ after correction for video section 1 was quite good, because the average number of in-focus objects present in this section was very close to 1 per field. However, for video section 2 , the average in-focus objects were close to 1.5 per field. Since a field cannot have 1.5 in-focus objects, the same correction factor was used for both sections. Not surprisingly, even after correction, $P_{d}$ was still relatively low in video section 2 . The corrected results are shown in Tables 2.3, and 2.4. It is worth mentioning that this problem would be vanished with a computer having a faster hard drive (the computer used in the test was a $1 \mathrm{GHz}$ Dell, circa 2000). Furthermore, such a dense patch of Phaeocystis was not usual for the focus detection program. The average in-focus object rate in most field applications was less than 1 per second compared to more than 60 per second in this case.

Table 2.3: Comparison of focus detection algorithms from AN9703, high magnification camera, video section 1 after correction. The numbers are blob counts; probability of detection $P_{d}$ and probability of false alarm $P_{f}$ are given as percentages.

\begin{tabular}{||l|l|l|l|r||}
\hline \hline Methods & In-focus & Out-of-focus & $P_{d}$ & $P_{f}$ \\
\hline Manual count & 132 & 808 & NA & NA \\
\hline A1 & 111 & 16 & $84 \%$ & $2.0 \%$ \\
\hline A2 & 120 & 18 & $91 \%$ & $2.2 \%$ \\
\hline A3 & 123 & 21 & $93 \%$ & $2.6 \%$ \\
\hline \hline
\end{tabular}

Two video sections of the low-magnification camera from cruise HALOS, Cape Cod Bay, March 1996, were also used to test the focus detection algorithms. Again, the videos were dubbed from SVHS to BETACAM-SP. In this tape, very high concentrations of Pseudocalanus with eggs were observed. Five second intervals of video were examined by a human operator since the concentration of the Pseudocalanus was not as high as the Phaeocystis. The manual counting process and post-processing by the focus-detection algorithm were the same as described above. The results are given 
Table 2.4: Comparison of focus detection algorithms from AN9703, high magnification camera, video section 2 after correction. The numbers are blob counts; probability of detection $P_{d}$ and probability of false alarm $P_{f}$ are given as percentages.

\begin{tabular}{||l|l|l|l|r||}
\hline \hline Methods & In-focus & Out-of-focus & $P_{d}$ & $P_{f}$ \\
\hline Manual count & 169 & 698 & NA & NA \\
\hline A1 & 131 & 13 & $77 \%$ & $1.9 \%$ \\
\hline A2 & 142 & 24 & $84 \%$ & $3.4 \%$ \\
\hline A3 & 131 & 17 & $77 \%$ & $2.4 \%$ \\
\hline \hline
\end{tabular}

in Tables 2.5, and 2.6. The relative low value of $P_{d}$ in Table 2.6 was due to a high number of in-focus objects. The process of writing files affected the performance of the algorithms, again correctable by a computer with faster hard disk drive.

Overall, all three algorithms did quite a good job on picking up in-focus objects, while rejecting out-of-focus objects. The algorithms that took the gradient strength into account (A2 and A3) worked a little better than the algorithm that thresholded gradient information. Between the two strategies of brightness correction, the additive worked as well as the multiplicative. Different parameter settings on the GUI (Fig 22 ) trade-off between $P_{d}$ and $P_{f}$. Since there were much higher numbers of out-of-focus objects than in-focus objects on the video, the outcome of focus detection algorithm was more sensitive to changes in $P_{f}$ than $P_{d}$. Another way to look at this issue is to check the percentage of in-focus objects from the outcome of each algorithm. For example, in Table 2.6, of 132 images chosen by A3 to be in-focus, 107 images were truly in-focus. That is to say, $81 \%$ of the output from A3 was true positive. A low true positive rate will increase the difficulty level of the subsequent classification problem and waste computational resources and disk space. On the other hand, a high true positive rate may result in undersampling the underlying population of plankton.

The manual counting process only counted the number of in-focus objects and outof-focus objects on each field. For each algorithm, the output images were examined by the same human operator in order to decide how many objects were in-focus and out-of-focus. The whole process was subjective. For each object, the image was not co-registered from the video to output images of each algorithm. The coregistration of every single object would be labor intensive. However, by only counting 
Table 2.5: Comparison of focus detection algorithms from HALOS, low magnification camera, video section 1 . The numbers are the blob counts; probability of detection $P_{d}$ and probability of false alarm $P_{f}$ are given as percentages.

\begin{tabular}{||l|l|l|l|r||}
\hline \hline Methods & In-focus & Out-of-focus & $P_{d}$ & $P_{f}$ \\
\hline Manual count & 116 & 597 & NA & NA \\
\hline A1 & 89 & 23 & $77 \%$ & $3.9 \%$ \\
\hline A2 & 98 & 16 & $84 \%$ & $2.7 \%$ \\
\hline A3 & 107 & 25 & $92 \%$ & $4.2 \%$ \\
\hline \hline
\end{tabular}

Table 2.6: Comparison of focus detection algorithms from HALOS, low magnification camera, video section 2. The numbers are the blob counts; probability of detection $P_{d}$ and probability of false alarm $P_{f}$ are given as percentages.

\begin{tabular}{||l|l|l|l|r||}
\hline \hline Methods & In focus & Out focus & $P_{d}$ & $P_{f}$ \\
\hline Manual count & 161 & 736 & NA & NA \\
\hline A1 & 106 & 32 & $66 \%$ & $4.4 \%$ \\
\hline A2 & 110 & 28 & $68 \%$ & $3.8 \%$ \\
\hline A3 & 121 & 30 & $75 \%$ & $4.1 \%$ \\
\hline \hline
\end{tabular}

the number of in-focus and out-of-focus objects, additional error was introduced by self-inconsistency. Nevertheless, this was the first quantitative study of focus detection algorithms. A correction factor is needed to interpret the focus detection output in the regions of extremely high plankton concentration.

\subsection{Conclusion}

A very large amount of data collected from an image-forming plankton sampler requires an automatic focus detection program to extract only in-focus objects from video. In this chapter, three algorithms were developed and tested on four video sections from VPR. This was the first quantitative study of focus detection program algorithms. In general, the algorithms have good performance for extracting in-focus objects without extracting too many out-of-focus objects. However, care is needed to interpret the focus detection output, especially in the regions of extremely high plankton concentration. 


\section{Chapter 3}

\section{Classification Method: Analysis}

\section{and Assessment}

A learning vector quantization neural network (LVQ-NN) classification system with combined shape-based features has been investigated. The objective of this study was to fully understand how the LVQ-NN classification system behaved on the fieldcollected plankton data. Multiple factors such as classifier complexity, number of training samples, quality of training samples, feature length, and presentation order of training samples have been examined. Three different methods have been proposed and implemented to improve the LVQ-NN classifier. This study suggested that the LVQ-NN classification system was very robust to varied parameter changes. However, for shape-based features, there was very limited improvement on classifying fieldcollected plankton images. The big classification performance difference between this study and previous studies indicated that previously reported accuracy of LVQ-NN was optimistically biased. Part of the results in this chapter was published in Marine Ecology Progress Series[34].

This chapter is organized as follows. In Section 3.1, I describe a state-of-the-art LVQ-NN classification system developed by Tang [150]. This system is well accepted but not well assessed. In Section 3.2, I investigate this system by changing classifier complexity, feature length, numbers of training samples, initial neuron position,

presentation order of training samples, different training samples and classification 
stability. In Section 3.3, I develop a two-pass classification system based on this LVQ-NN classification system. In Section 3.4, I propose a method to correct the bias of the classification system. In Section 3.5, I develop a distance rejection metric on LVQ-NN classification system. Part of the results discussed in this chapter was published in Davis et al.[34]

\subsection{System overview}

\subsubsection{Artificial Neural Networks}

Artificial Neural Networks (ANN) have experienced three periods of extensive activity. The first peak in the 1940s was due to McCulloch's pioneering work [113]. The second in the 1960s was Rosenblatt's perceptron convergence theorem [136]. Minsky [116] showed that a single perceptron was not able to solve a simple XOR problem. Such limitation dampened the progress in ANN. The third peak was due to the Hopfield's energy approach [70] and back-propagation learning algorithm for multilayer perceptrons by Werbos [167], and later popularized by Rumelhart [137].

The great benefits of the ANN are the simplicity of the learning algorithm, the ease in model selection, and incorporation of heuristic information and constraints. ANN has been widely used in feature extraction [110, 105], character classification [97, 98], speaker identification [124], and general object classification [148, 150].

\subsubsection{Learning vector quantization neural network classifier}

Learning vector quantization (LVQ) is a supervised version of vector quantization. Its objective is to learn a set of prototypes (codebooks) which best represent each class. We implement it with an artificial neural network [150,34]. The neural network has two layers, namely a competitive layer and a linear output layer. The complexity of the neural network (prototypes of subclass, number of neurons) is based on the number of training samples and the number of classes in the classifier. The number of output layer neurons is equal to the number of taxa. The weights of the neurons 
for each class are initialized to be the mean of the training feature vectors for that class plus a small random value. The network is trained by randomly presenting the training samples to the network. Each training sample is classified by the current LVQ neural network. Depending on the outcome of the classifier, the weights of the neurons are adjusted in the following two ways: If the predicted label of a sample agrees with its true label, the weights of the winning neuron (prototype) are updated in such a way that the winning neuron moves a step closer to the training sample in the feature space; otherwise, the weights of the winning neuron are updated such that the winning neuron is pushed a step away from the training sample in the feature space. The training process stops when the preset goal or the maximum training time is reached. The trained network is saved as the final classifier.

\subsubsection{Principal component analysis}

Principal Component Analysis (PCA) is widely used in signal processing, statistics, and pattern recognition [84]. Denote $\mathbf{x}=\left(x_{1}, x_{2}, \cdots, x_{n}\right)^{T}$ as a $n$-dimensional original feature vector, and $\mathbf{y}=\left(y_{1}, y_{2}, \cdots, y_{m}\right)^{T}$ as a $m$-dimensional final feature vector $(m \leq n)$, PCA seeks a linear transformation $\mathbf{T}$, such that

$$
\mathbf{y}=\mathbf{T x}
$$

where $\mathbf{T}$ is $m \times n$ matrix. The main idea of the transformation is to explain the maximum amount of variance in $n$-dimensional vector $\mathbf{x}$ by a much lower dimensional vector $\mathbf{y}$. In other words, PCA seeks a linear projection that best represents the data in the mean-square sense.

In order to find the transformation matrix $\mathbf{T}, p$ observations of $\mathbf{x}$ ( $p$ training samples, $p \geq n$ ) are required. First, the $n$-dimensional mean vector $\mu$ and $n \times n$ covariance matrix $\boldsymbol{\Sigma}$ are computed from all the training samples. Next, the eigenvectors and eigenvalues are computed from the covariance matrix, and sorted according to decreasing order of eigenvalue. Denoting these eigenvectors as $\mathbf{e}_{\mathbf{1}}, \mathbf{e}_{\mathbf{2}}, \cdots, \mathbf{e}_{\mathbf{n}}$ and corresponding eigenvalues as $\lambda_{1}, \lambda_{2}, \cdots, \lambda_{n}$, and choosing the $m$ eigenvectors having 
the largest eigenvalues, we form an $m \times n$ matrix $\mathbf{T}$ whose rows are transposes of the $m$ eigenvectors. The representation of data by PCA projects the data onto the $m$-dimensional subspace according to

$$
\mathbf{y}=\mathbf{T}(\mathbf{x}-\mu) .
$$

\subsubsection{Feature extraction}

For each sample image, four different groups of feature presentation methods are used, which include 7 moment invariants, 64 Fourier descriptors, 160 pattern spectra, and 6 morphological measurements. These features are combined into a single feature vector with 237 elements. All the feature elements are first normalized to zero mean and unit standard deviation. Principal component analysis is then applied on this feature vector to eliminate linear dependence among elements of the feature vector. The 20-30 largest eigenvalues account for nearly all the variances in feature space of the training samples. The corresponding eigenvectors are saved and used as a transformation matrix. All the non-training samples are normalized and projected onto these 20-30 orthogonal bases via the transformation matrix. The resulting feature vector is the input of the LVQ neural network classifier.

\subsubsection{Classification performance estimation}

After a classifier has been built, its classification generalization performance (performance from a set of independent samples) needs to be evaluated. For finite sample sizes and unknown class-conditional distribution, the only way to estimate the generalization performance is to use an empirical method. There are three empirical ways to estimate the generalization performance. The first approach is often called the resubstitution method, which involves classifying all the training samples, and uses classification accuracy on training samples as generalization performance. It is fast and does not require extra labeled samples. Nevertheless, this method has an

optimistically biased estimate of classification performance. 
The second approach is often called the cross-validation method, which can be further divided into three cases. The first case is often called holdout method, which uses a completely independent test data set to evaluate generalization performance of a classifier. The drawback of this method is that it requires twice as many labeled samples as resubstitution. According to Jain et al. [77], this estimate is pessimistically biased. From the results in this chapter, I do not get any pessimistically biased estimates. I used the holdout method as a classification performance estimate from the whole data set. Since there is an overlap between training samples and test samples, strictly speaking, it is a misnomer. However, the overlap is small and the difference between training accuracy and test accuracy of the classifier is also small. I argue that the difference between the "true" holdout and my pseudo-holdout is negligible.

The second case of the cross-validation method is often called the leave-one-out method, which involves building $n$ classifiers with $n-1$ training samples. Each time, a different sample is left out to build a classifier and used to test the classifier. Here $n$ is the number of total training samples. The leave-one-out method is computation demanding, and it has an unbiased estimate with large variance [77]. The third case of the cross-validation method is the rotation method, also called an n-fold cross validation method, which is a compromise between the holdout and leave-one-out methods. It divides the training samples into $p$ disjoint subsets, using $p-1$ subsets for training a classifier and the remaining subset for testing the classifier.

The third approach is called the bootstrap method, which involves generating multiple bootstrap sample sets of size $n$ by sampling all the training samples with replacement. The bootstrap bias and variance estimate can be estimated from bootstrap sample sets. When the number of bootstraps approaches infinity, the bootstrap variance becomes the traditional variance of mean [42]. In this chapter, the resubstitution, leave-one-out, and holdout methods are used to estimate classification performance. 


\subsection{Assessment Result}

\subsubsection{Classifier complexity vs. classifier performance}

The relationship between classifier performance and classifier complexity is investigated first. The classifier complexity is characterized by the number of neurons per taxon, which governs the expressive power of the neural network. The neurons are evenly distributed among taxa. Classifiers with 3 neurons per taxon up to 100 neurons per taxon are trained with the same amount of training samples. The training samples come from a mixture of four VPR tows from the same cruise [34]. Each classifier is applied to all the images from a single VPR tow, which includes more than 20,000 images. Classification accuracy is obtained by comparing the predicted classification label with the human label for each image. The classification accuracy rises from 3 to 4 neurons per taxon, reaches its peak at 10 to 15 neurons per taxon, and then hovers around with an overall accuracy of 59-60\% (Figure 3-1). No obvious over-training effect is observed. This can be seen more clearly in Figure 3-2. However, when a large number of neurons is used, the classifier takes a long time to train. Furthermore, when classification performance is inferred from training accuracy (e.g. resubstitution method), using a large number of neurons can result in a large bias on the classification accuracy estimation (for example, Tang $[148,150]$ used an average of training and test accuracy to compare classification performance).

\subsubsection{Feature length versus classification performance}

Final feature length may play an important role in classification performance. Choosing a short feature length may lose the discriminative power of the feature set, while choosing a long feature length may include noise to degrade classification performance. In this study, feature lengths from 2 to 40 are examined. Again, classifiers are trained from a mixture of four VPR tows from the same cruise. A total of 70 neurons are used to train the classifiers, which are evenly divided into 7 taxa with 10 neurons per taxa. The classification performance of different taxa varies differently with the 

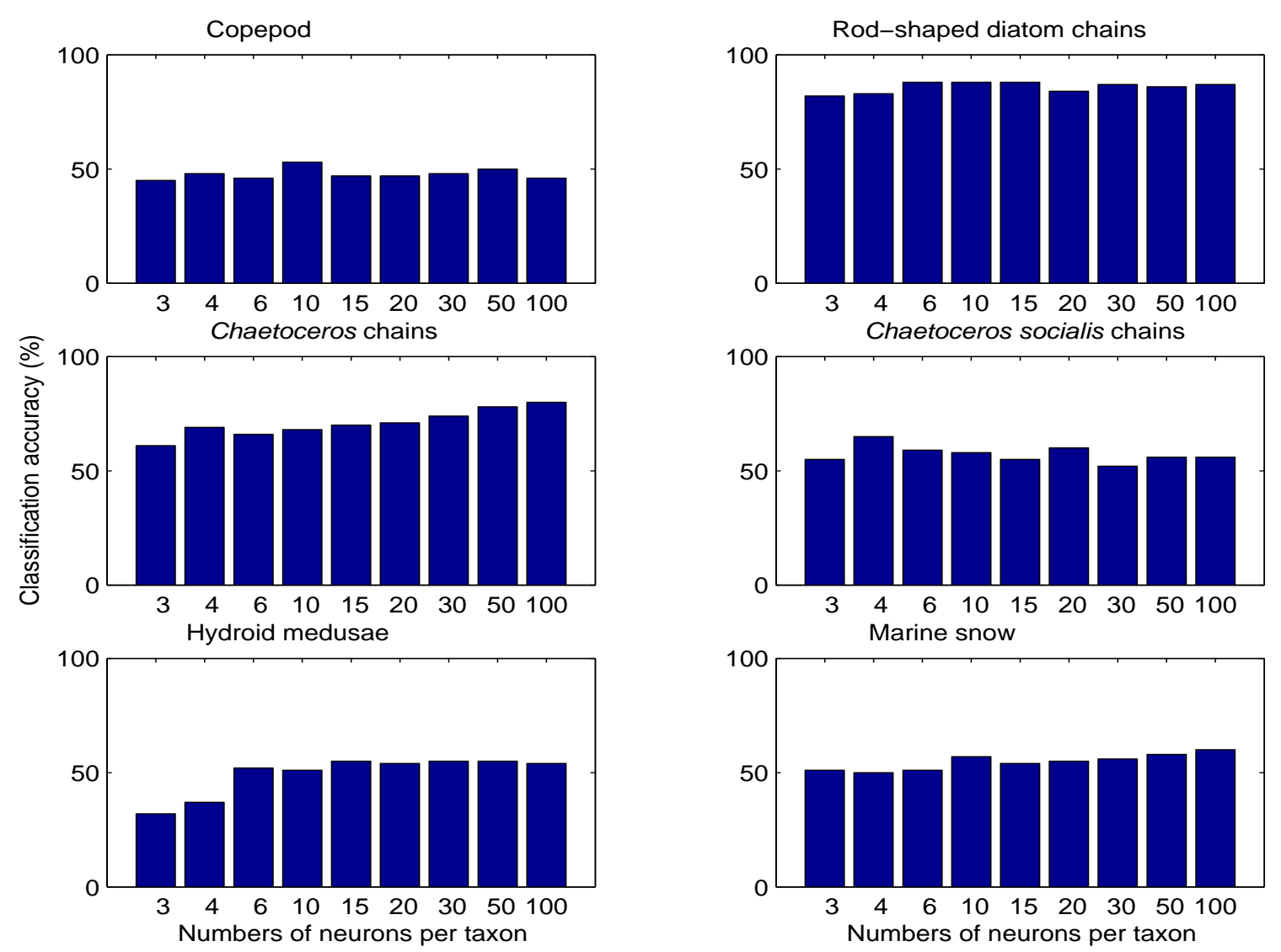

Figure 3-1: Classification performance with respect to classifier complexity.

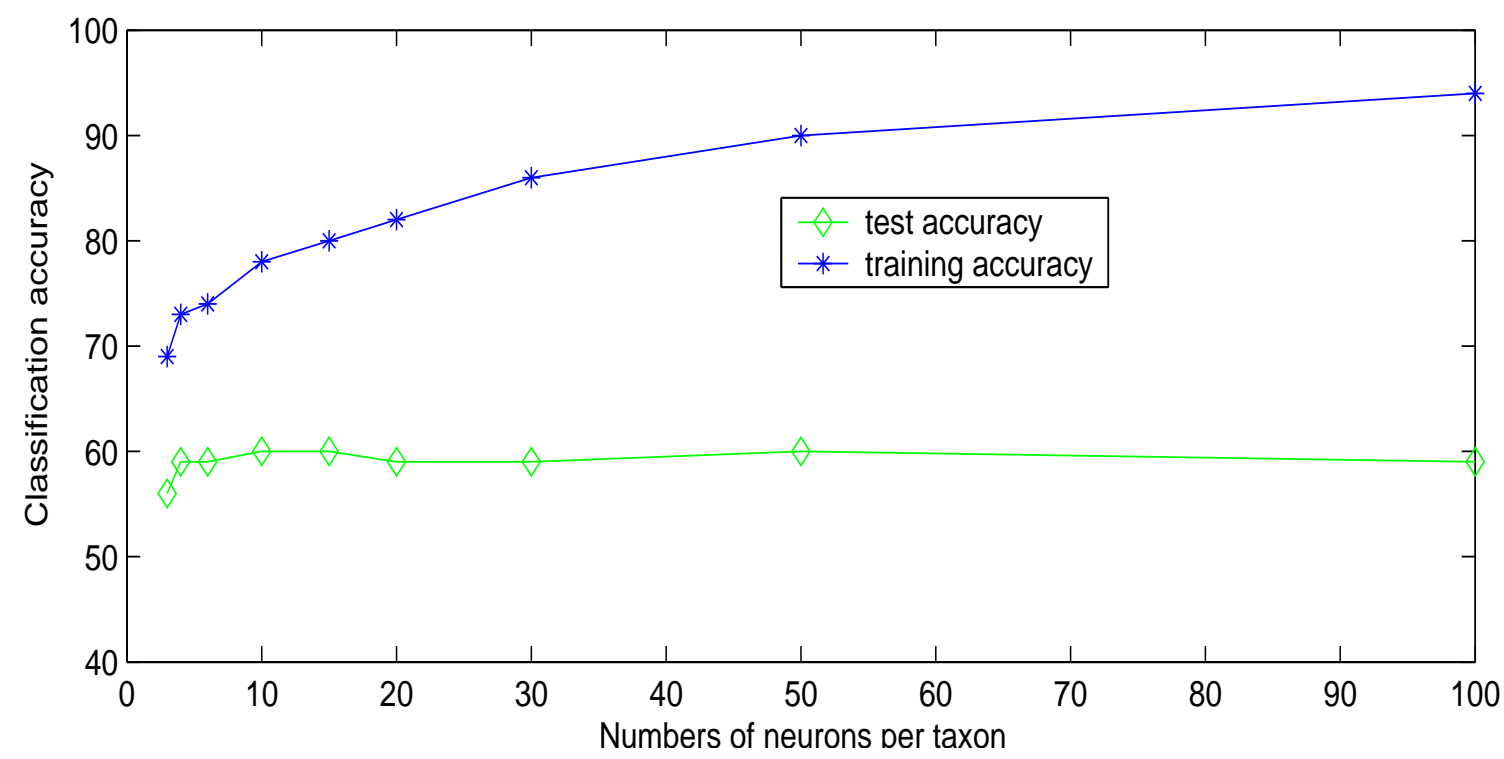

Figure 3-2: Training and test accuracy with respect to classifier complexity. 
change of feature length (Figure 3-3). Some taxa have relatively steady classification performance, while the others have more variations with the change of feature length. However, the overall classification performance (average over all the taxa) is fairly steady and reveals a slight increase with increasing feature length (Figure 3-4). The steady increase of training accuracy with feature length suggests that extra features capture training sample specific features instead of general features of each taxon. On the other hand, the test accuracy curve is fairly flat from the feature lengths from 20 to 40 (Figure 3-4).
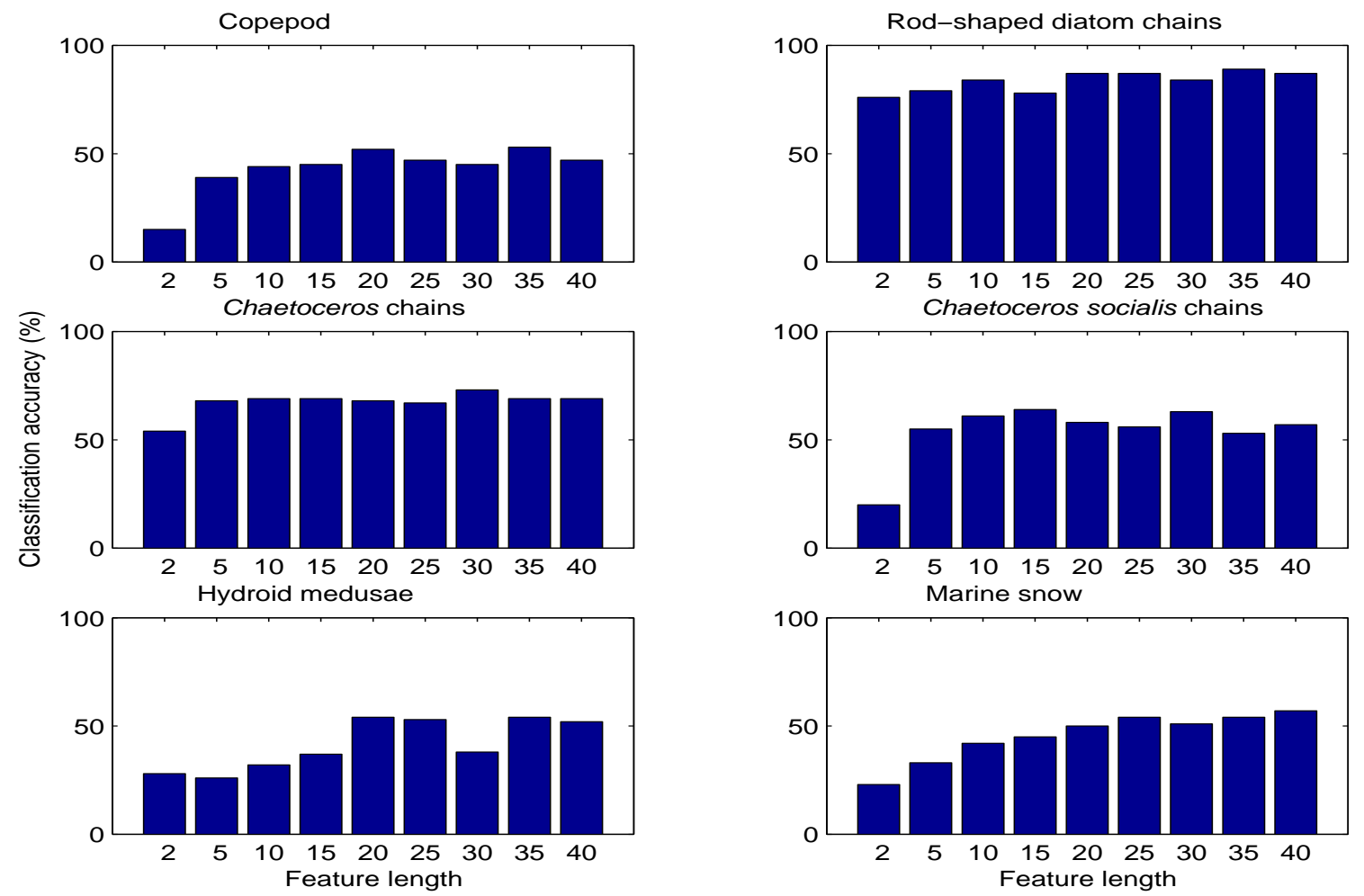

Figure 3-3: Classification performance as a function of feature length for each taxon.

\subsubsection{Learning curve - numbers of training samples versus classifier performance}

The number of training samples is an important factor for supervised learning. Few training samples may not fully present the feature space, while a large number of 


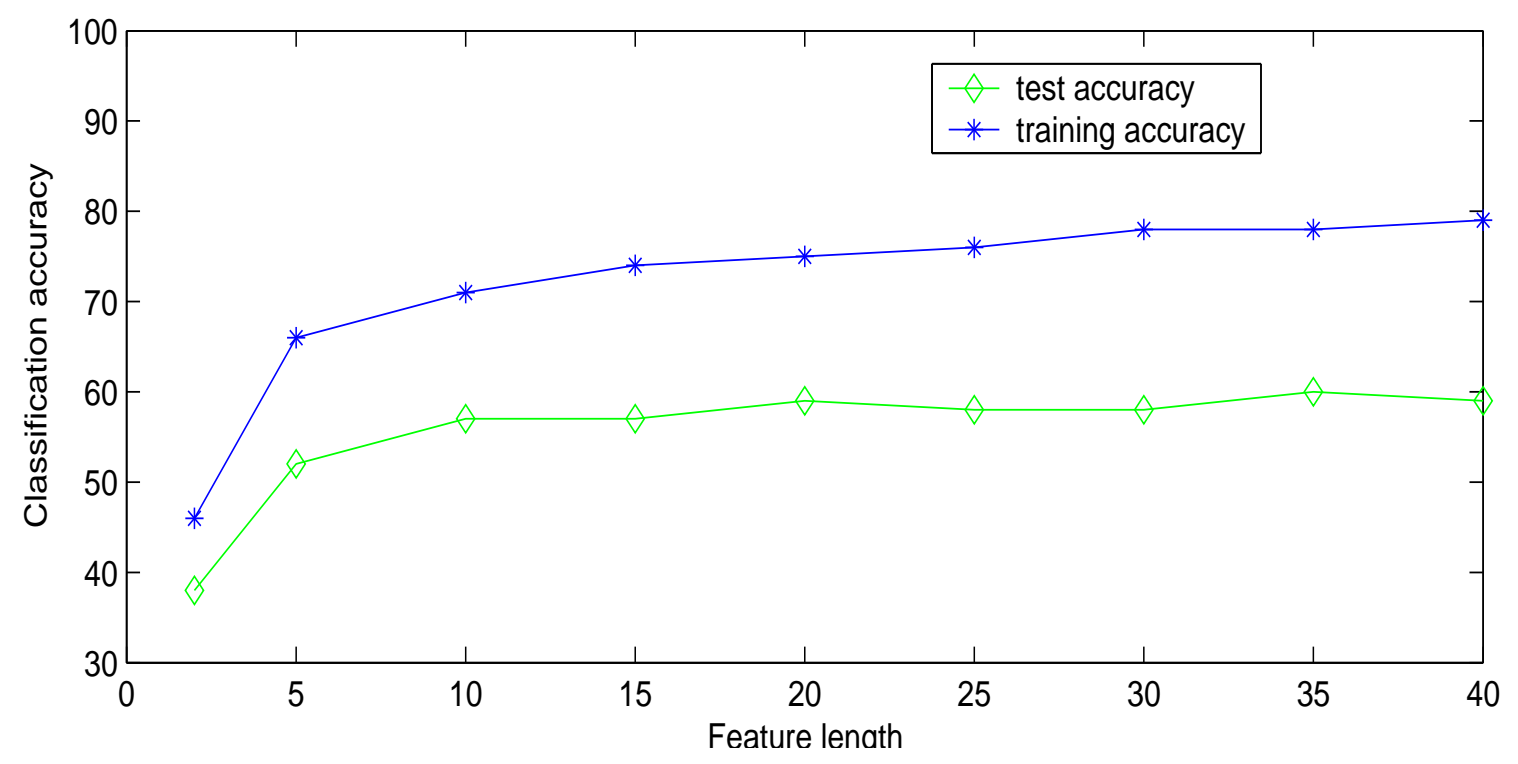

Figure 3-4: Training and test accuracy with respect to feature length.

training samples are very costly to get because labeling requires extensive expert time. Training on a very large data set is also computationally intensive, which may take days or even months. In this study, the relationship between the number of training samples and classification performance is explored empirically. The objective is to understand how many training samples are "good" enough in the sense of manual labeling efficiency. Training samples are randomly selected from the whole data set. The classification performance as a function of training sample size for each taxon is shown in Figure 3-5. In general, classification performance tends to increase with more training samples being available. For copepod and rod-shaped diatom chains, classification accuracy remains almost the same from 50 samples per taxon to 400 samples per taxon. For other taxa, classification accuracy increases with more training samples added. Compared to Figure 3-1 and Figure 3-5, there are significant differences of classification accuracy for copepod and rod-shaped diatom chains. Such differences are caused by different training samples used. I will discuss more on the training samples effect later in this chapter. Figure 3-6 shows training and test classification accuracy with respect to training sample size (learning curve). From 50 to 200 training samples per taxon, the test classification accuracy has an increase of 
$4 \%$ with respect to an increase of 100 samples per taxon. From 200 training samples to 400 training samples per taxon, the increase of test classification accuracy drops down to $0.5 \%$ with an increase of 100 samples per taxon. I conclude that 200 training samples per taxon is the optimal number of training samples in terms of manual labeling efficiency. Hereafter, 200 training samples per taxon are used if it is not explicitly stated. However, as shown in Figure 3-5, the optimum training samples per taxon is taxon dependent. For relatively "easy" taxon such as rod-shaped diatom chains, a small number of training samples are sufficient. On the other hand, for really "hard" taxon such as copepods, considering large within-taxonomic group variation of copepods, such difference in training sample size has small effect on classifcation accuracy.
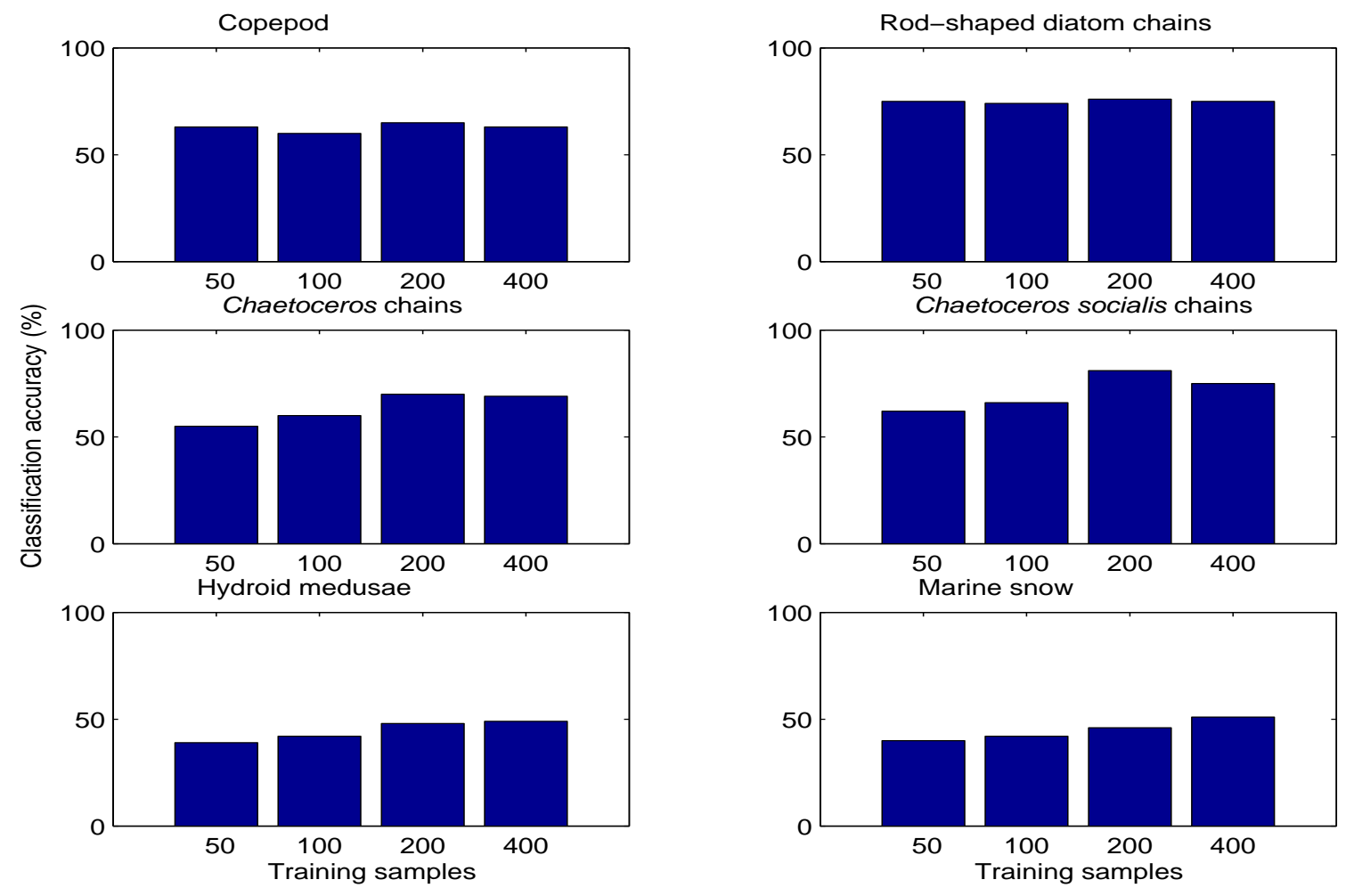

Figure 3-5: Classification performance as a function of training sample size for each taxon. 


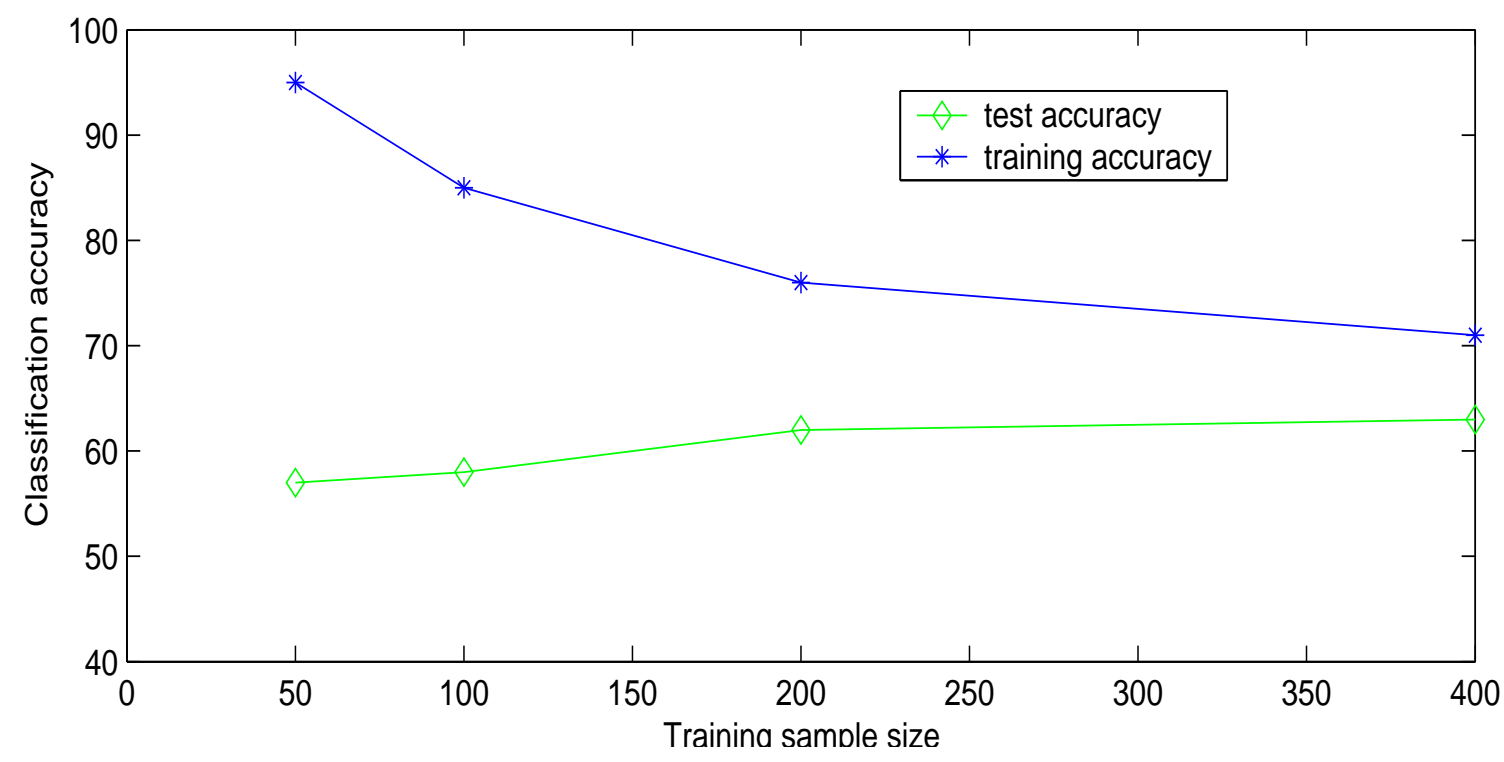

Figure 3-6: Training and test accuracy with respect to training sample size.

\subsubsection{Initial neuron position versus presentation order of train- ing samples}

There are two sources of randomness when the classifiers have been trained with the same training samples. The first one is the initial positions of neurons before the training processes start. The second one is the presentation orders of the training samples to the classifiers. Both randomized initial position of neurons and presentation order of training samples are used in order to speed up the learning process. In this section, I investigate which source of randomness may have the largest impact on classification performance. Two sets of tests are conducted. In both sets of tests, each classifier is built on the same training samples with 200 training samples per taxon randomly selected from the whole data set. For simplicity, the resubstitution method is used to evaluate classification accuracy. Since the classification performance is compared in the relative sense, I have used training accuracy as a classification performance indicator. The mean and standard deviation of training accuracy are calculated from 10 different trials. The difference between the first set of tests and the second set of tests is that in the first set of tests each classifier has both different initial position of 
neurons and different presentation order of training samples, while in the second set of tests each classifier starts with same initial position of neurons and is trained with different presentation order of training samples. The result is shown in Figure 3-7. The mean and standard deviation of the classification performance are almost identical, which suggests that different initial positions of neurons have little effect on the final classifiers. This agrees a well known result that the random presentation order of training samples has more impact on classification performance.
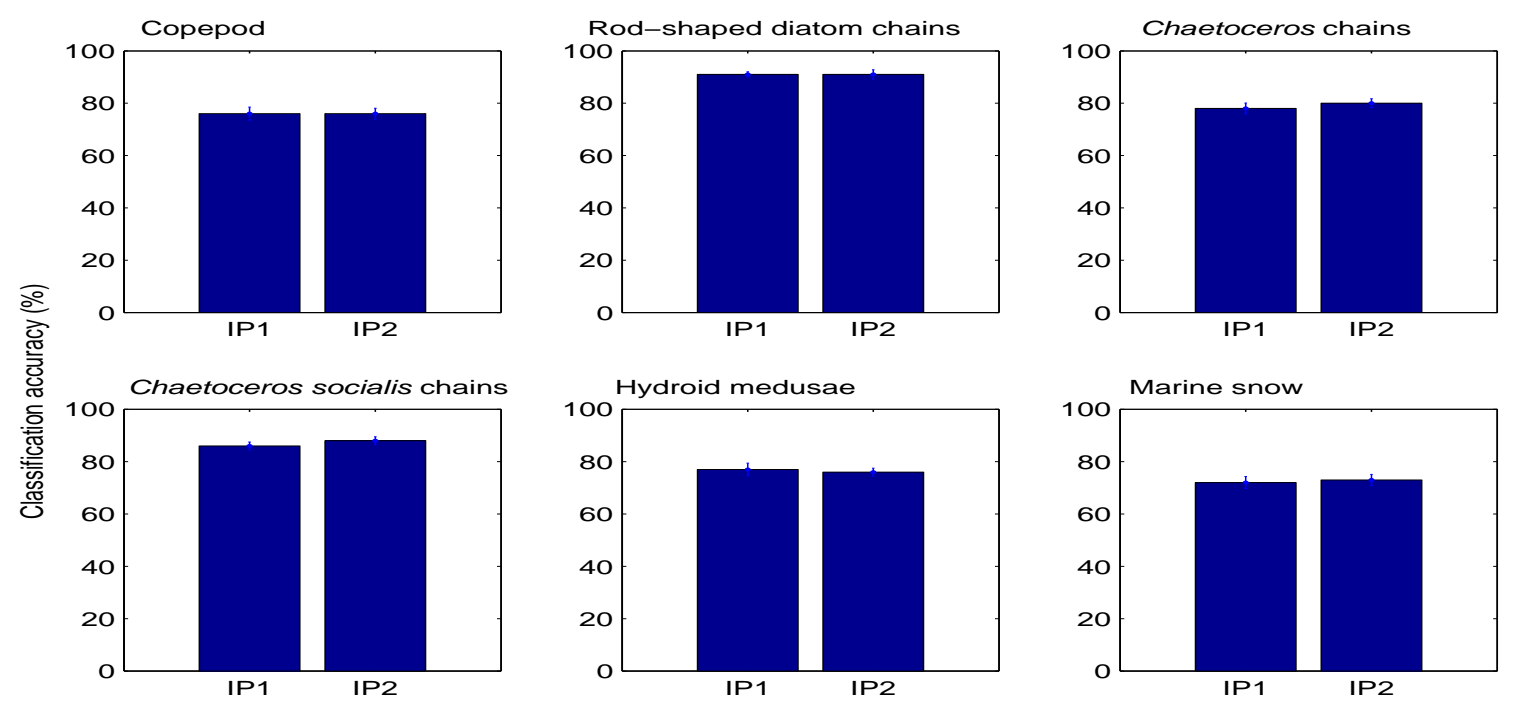

Figure 3-7: Comparison between the random initial position of neurons and random order of presentation order of training samples. IP1 - different initial position of neurons, random representation order of training samples; IP2 - same initial position of neurons, random representation order of training samples

\subsubsection{Training samples effect}

Classifiers are not only affected by the size of the training samples, but also by the quality of the training samples. We have already seen from Figure 3-1 and Figure 3-5 that different training samples significantly affect the classification accuracy of copepod and rod-shaped diatom chains. In this section, we try to quantify classification performance variations from different training samples. Three sets of tests have been 
conducted for this manner. For all the tests, training samples are randomly selected from the whole data set with 200 samples per taxon. In the first set of tests (TS1), each classifier is built from different training samples, and is then evaluated by the leave-one-out method. In the second set of tests (TS2), each classifier is also built from different training samples, and is evaluated by the holdout method. In the third set of tests (TS3), each classifier is built from the same training samples, and evaluated by the holdout method. The results are shown in Figure 3-8. It is interesting to see that the leave-one-out method has high estimates on certain taxa such as rodshaped diatom chain and hydroid medusae, while it has low estimates on other taxa such as copepod and Chaetoceros chains. This does not agree with the statement that the leave-one-out estimate is unbiased and the holdout estimate is pessimistically biased by Jain et al. [77]. The overall classification accuracy is very close between the leave-one-out method and the holdout method, given that training samples are randomly selected from the whole data set. Otherwise, the cross validation (leaveone-out) method may still have a biased estimate of classification accuracy [34]. In general, the variation of classification accuracy (variance of mean accuracy) is much smaller when classifers are trained by a single set of training samples than different sets of training samples. Such variation is also taxon dependent. For "easy" taxon such as rod-shaped diatom chains, the variation is much smaller compared to "hard" taxon such as copepods. The variation of the leave-one-out method is similar to that of the holdout method.

\subsubsection{Classification stability}

The stability of a classfier, namely, how the classifier is affected by changing the training samples, has been used to study generalization performance of the classfier by many researchers theoretically $[13,43]$. In this section, I have investigated stability of our LVQ-NN classifier in terms of variance of abundance estimation of each taxon. Nine classifiers are built from different random sets of training samples, which contain 200 samples for each taxon, and are randomly picked from the whole data set. Each classifier is then used to classify the whole data set. The mean and standard deviation 

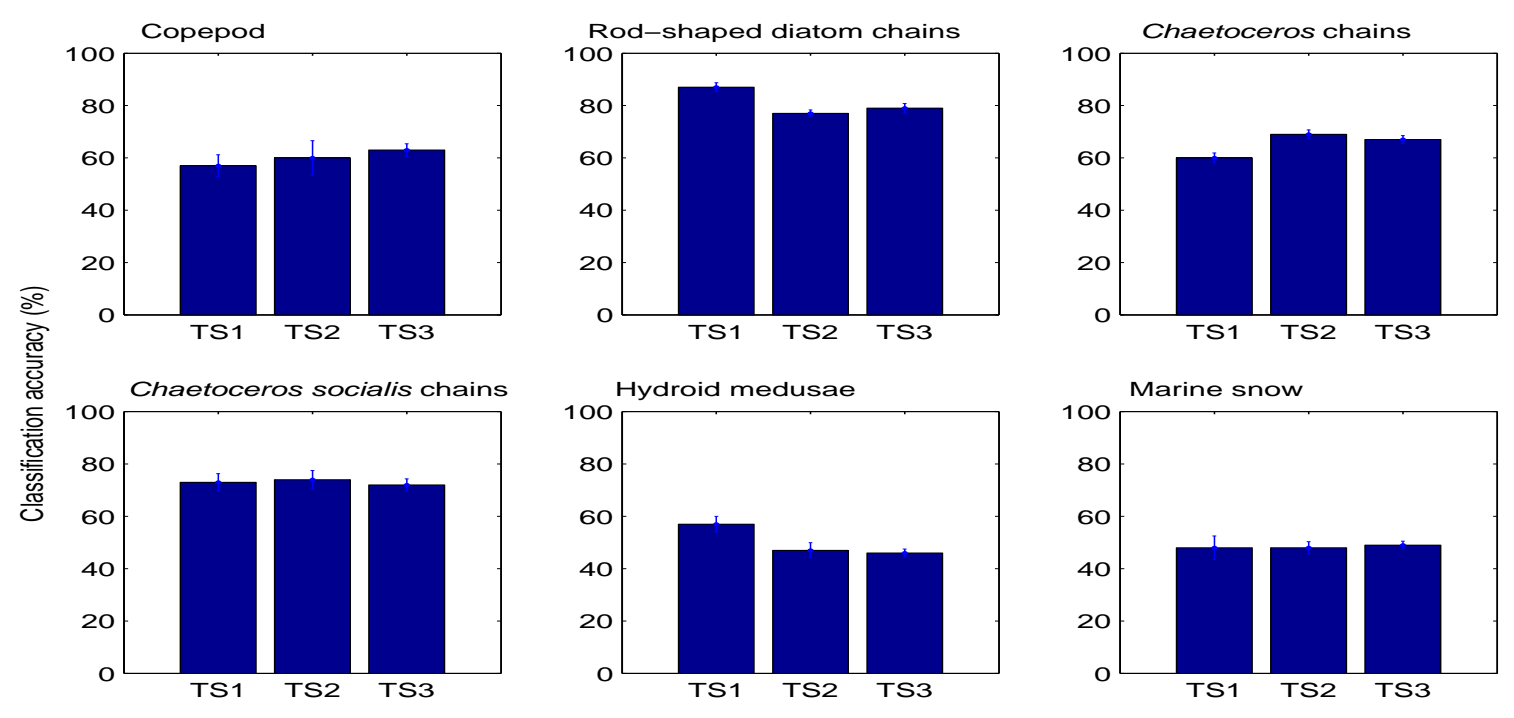

Figure 3-8: Comparison of different training samples effect on classfication performance. TS1 - different sets of training samples, leave-one-out method; TS2 - different sets of training samples, holdout method; TS3 - single set training samples, holdout method

abundance are calculated. The mean, upper and lower limit of $95 \%$ confidence interval abundances are ploted against manually sorted abundance (Figure 3-9). Most taxa have stable classification results except copepods, which show a large difference between the upper and lower limit of $95 \%$ confidence interval.

\subsection{Two-pass classification system}

When a classifier is used to estimate abundance, there are two sources which make the estimation biased. The first source is the relative abundance of each taxon. The classifier tends to underestimate the relative high abundance taxon and overestimate the relative low abundance taxon. For example, suppose that a sample contains 2 taxa, with 90 individuals of one taxon and 10 individuals of the other taxon. For both taxa, the classifier has the probability of detection of $90 \%$. The expected number of individuals classified as the first taxon is $(90 \times 0.9)+(10 \times 0.1)=82$ and the expected number classified as the second taxon is $(10 \times 0.9)+(90 \times 0.1)=18$. Despite the 

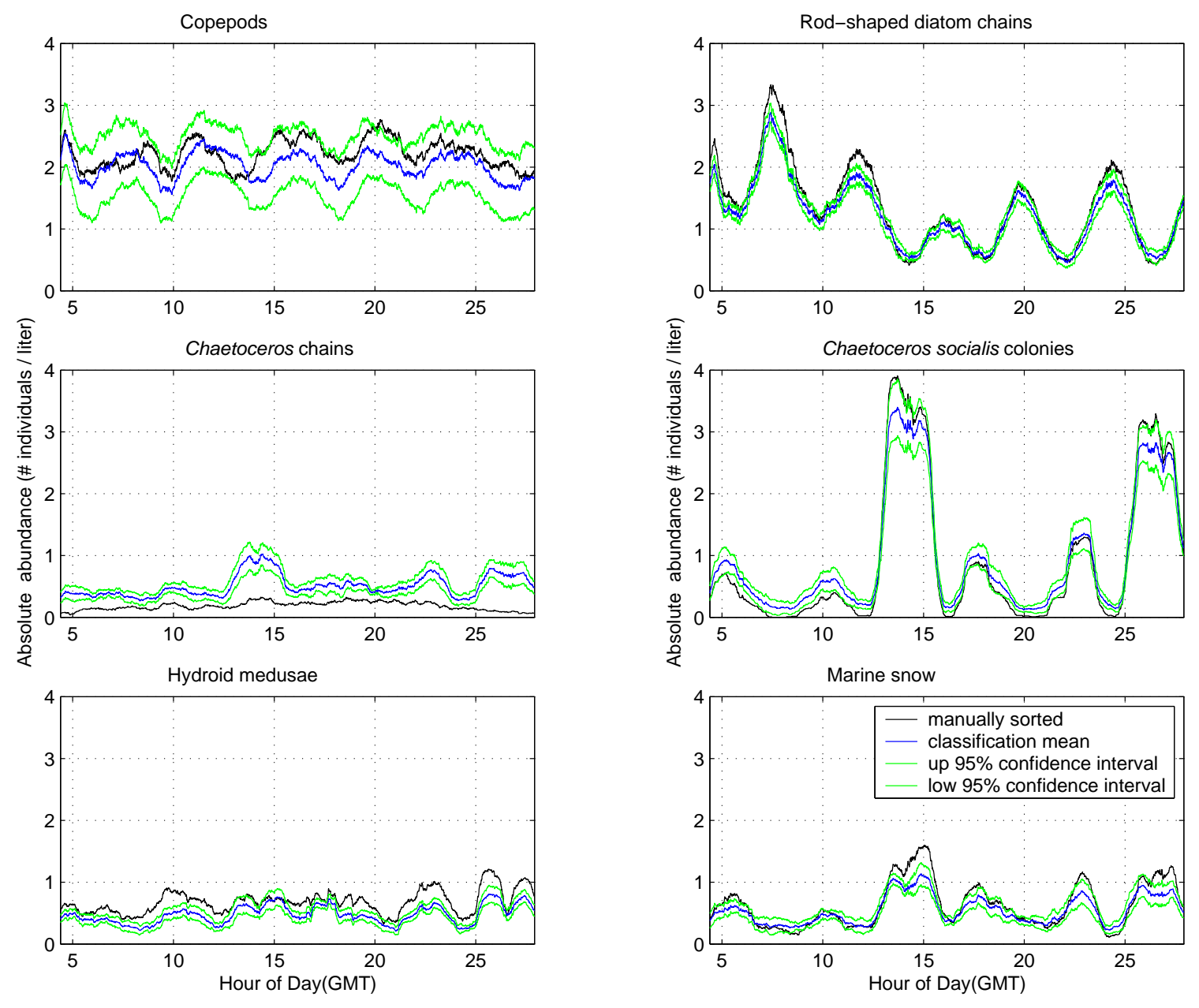

Figure 3-9: Mean, upper and lower limit of 95\% confidence interval of abundance estimates from LVQ-NN classifiers and that of manually sorted results. Time series abundance plots along the tow path are shown for 6 dominant taxa. Data were first binned in 10 second time intervals, and a one-hour smoothing window was applied to the binned data. 
classifier having a relative high probability of detection of $90 \%$, the abundance of the rare taxon in the sample is, on average, overestimated by a factor of nearly 2 . The second source is uneven probability of detection. It is easy to show in the two taxa case. Suppose that a sample contains 2 taxa, with 50 individuals of one taxon and 50 individuals of the other taxon. The classifier has probabilities of detection of $90 \%$ and $50 \%$ for each taxon, respectively. The expected number of individuals classified as the first taxon is $(50 \times 0.9)+(50 \times 0.5)=70$ and the expected number classified as the second taxon is $(50 \times 0.5)+(50 \times 0.1)=30$. Although two taxa are equally abundant, the classifier has overestimated the taxon with the higher probability of detection.

\subsubsection{Decision rules}

The above problem arises from uneven distribution and probability of detection. One way to overcome such a problem is to design a classifier under minimax criterion. Briefly speaking, one first searches for the prior for which the Bayes risk is maximum, one then finds the decision boundary to minimize the above Bayes risk. The solution is often called the minimax solution. Denoting that $\mathcal{R}_{1}$ is the region in feature space where the classifier decides $\omega_{1}$, and likewise $\mathcal{R}_{2}$ decides $\omega_{2}$, one can write the overall risk of the classifier in terms of conditional risks [42]:

$$
\begin{aligned}
R=\int_{\mathcal{R}_{1}}\left[\lambda_{11} P\left(\omega_{1}\right) p\left(\mathbf{x} \mid \omega_{1}\right)+\right. & \left.\lambda_{12} P\left(\omega_{2}\right) p\left(\mathbf{x} \mid \omega_{2}\right)\right] d \mathbf{x} \\
& +\int_{\mathcal{R}_{2}}\left[\lambda_{21} P\left(\omega_{1}\right) p\left(\mathbf{x} \mid \omega_{1}\right)+\lambda_{22} P\left(\omega_{2}\right) p\left(\mathbf{x} \mid \omega_{2}\right)\right] d \mathbf{x},
\end{aligned}
$$

where $P\left(\omega_{i}\right)$ is prior probability, $p\left(\mathbf{x} \mid \omega_{i}\right)$ is conditional probability, and $\lambda_{i j}$ is the loss function. If one uses the fact that $P\left(\omega_{1}\right)=1-P\left(\omega_{2}\right)$ and that $\int_{\mathcal{R}_{1}} p\left(\mathbf{x} \mid \omega_{1}\right) d \mathbf{x}=$ 
$\left.1-\int_{\mathcal{R}_{2}} p\left(\mathbf{x} \mid \omega_{2}\right)\right] d \mathbf{x}$, one can rewrite the above risk function as:

$$
\begin{aligned}
& \left.R\left(P\left(\omega_{1}\right)\right)=\lambda_{22}+\left(\lambda_{12}-\lambda_{22}\right) \int_{\mathcal{R}_{1}} p\left(\mathbf{x} \mid \omega_{2}\right)\right] d \mathbf{x} \\
& \left.\quad+P\left(\omega_{1}\right)\left[\left(\lambda_{11}-\lambda_{22}\right)+\left(\lambda_{21}-\lambda_{11}\right) \int_{\mathcal{R}_{2}} p\left(\mathbf{x} \mid \omega_{1}\right) d \mathbf{x}-\left(\lambda_{12}-\lambda_{22}\right) \int_{\mathcal{R}_{1}} p\left(\mathbf{x} \mid \omega_{2}\right)\right] d \mathbf{x}\right] .
\end{aligned}
$$

If one sets the second term at the right hand side of the above equation equal to zero, the risk function is independent of prior probabilities. Such a solution is called a minimax solution. When the zero-one loss function is used, i.e.,

$$
\lambda_{i j}= \begin{cases}0 & i=j \\ 1 & i \neq j,\end{cases}
$$

the condition of the minimax solution can be simplified as,

$$
\left.\int_{\mathcal{R}_{2}} p\left(\mathbf{x} \mid \omega_{1}\right) d \mathbf{x}-\int_{\mathcal{R}_{1}} p\left(\mathbf{x} \mid \omega_{2}\right)\right] d \mathbf{x}=0
$$

Another way is to use recursive prior estimation. Since in most real world problems, the form of the conditional probability distribution is complicated or even unknown, finding the decision boundary of the minimax solution is not trivial. In this section, we adopt the second approach, that is to say, we try to recursively estimate the priors.

The three most popular decision rules are illustrated in Figure 3-10. The maximum likelihood (ML) decision rule seeks the intersection between two conditional probability distributions, and the corresponding decision value is $x_{M L}^{*}$. The maximum a posteriori (MAP) decision rule seeks the intersection between two scaled conditional probability distributions, and the corresponding decision value is $x_{M A P}^{*}$. The scaling factor is the ratio of the priors of the two classes. The minimax decision rule seeks a decision value $x_{M M}^{*}$ which makes the areas under the two distribution tails equal.

In my application, I have used classification to estimate the fine resolution of priors for each taxon. Before classification, nothing is known about the priors, so a 


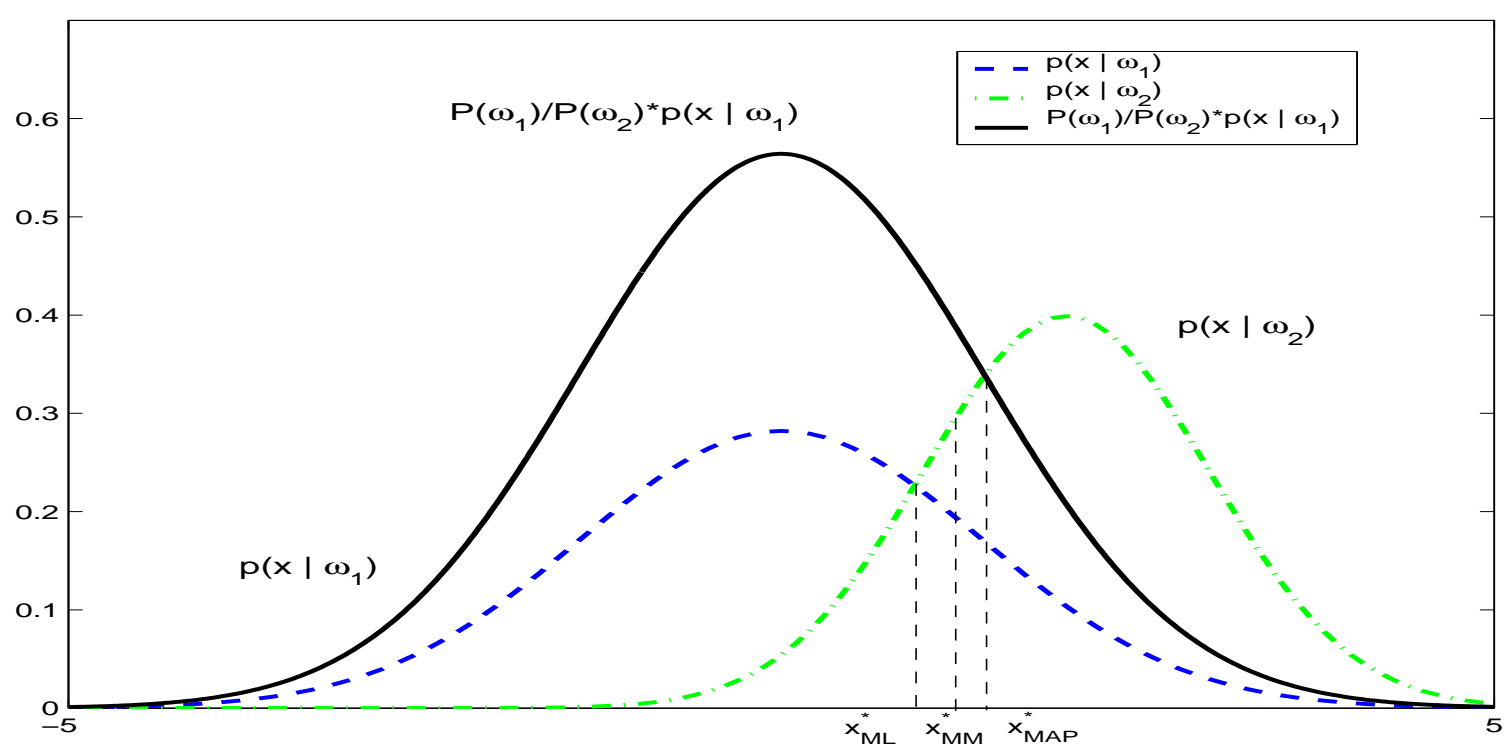

Figure 3-10: Illustration of the three most popular decision rules. $x_{M L}^{*}$ - maximum likelihood decision rule, $x_{M A P}^{*}$ - maximum a posteriori decision rule, $x_{M M}^{*}$ - minimax decision rule

ML decision rule is applied in the first classifier. Local priors can be estimated from the first classification results using a moving window average method (for example, calculate from the latest 100 samples). The priors estimated from the first classifier are then applied in the second classifier based on a MAP decision rule. I call such a system a two-pass classifier since each sample needs to be classified twice.

The structure of the two-pass classifier is shown in Figure 3-11. There are two classifiers involved in the two-pass classification system. The first classifier is the same as a single classifier, the outcomes of which are used to estimate local priors for each taxon. The predictions of the first classifier are collected by a prior estimator. After collecting a certain number of samples, local priors of each taxon will be reported by the prior estimator. These local priors are updated afterwards when a new sample is available. The second classifier utilizes the local priors as well as the same feature vector used in the first classifier to get a better prediction for each sample. For simplicity, the algorithms of the two classifiers are identical, the only difference is the priors of each taxon. Priors of the first classifier are set to uniform for all taxa, while priors of the second classifier are calculated from the prior estimator. The rationale 
of two-pass classification is that as long as the first classifier is better than a random guess, the priors estimated from it are much better than uniform priors. Given this piece of information, the second classifier will further improve the prediction of each sample beyond that of the first classifier.

\subsubsection{Implementation}

To summarize the above discussion, the two-pass classification system can be implemented in the following steps:

1. Train a LVQ neural network classifier with an equal number of training samples for each taxon.

2. Generate a confusion matrix with the leave-one-out method from training samples. Calculate the probability of detection $\left(P_{d}\right)$ for each taxon.

3. For each field sample, classify it with the classifier built above.

4. Set up a first-in-first-out (FIFO) queue. If the queue is not filled, output the predicted class label, and go to step 2. Otherwise update priors for each taxon.

5. Use the probability of detection to correct the priors.

6. Calculate the scaling factors $C\left(\omega_{i}\right)=\sqrt{\left(1-P\left(\omega_{i}\right)\right)}$ for each taxon based on their priors.

7. Use the second classifier to compute the distance map between the sample and neurons, scale the distance map with scaling factor $C\left(\omega_{i}\right)$.

8. Make the prediction based on the modified distance map and go back to step 2 .

\subsubsection{Results}

Abundance estimations of six dominant taxa are compared among manually sorted, single NN classifier and two-pass NN classifier with combined features of moment invariants, Fourier descriptors, pattern spectra and morphological measurements (Fig- 


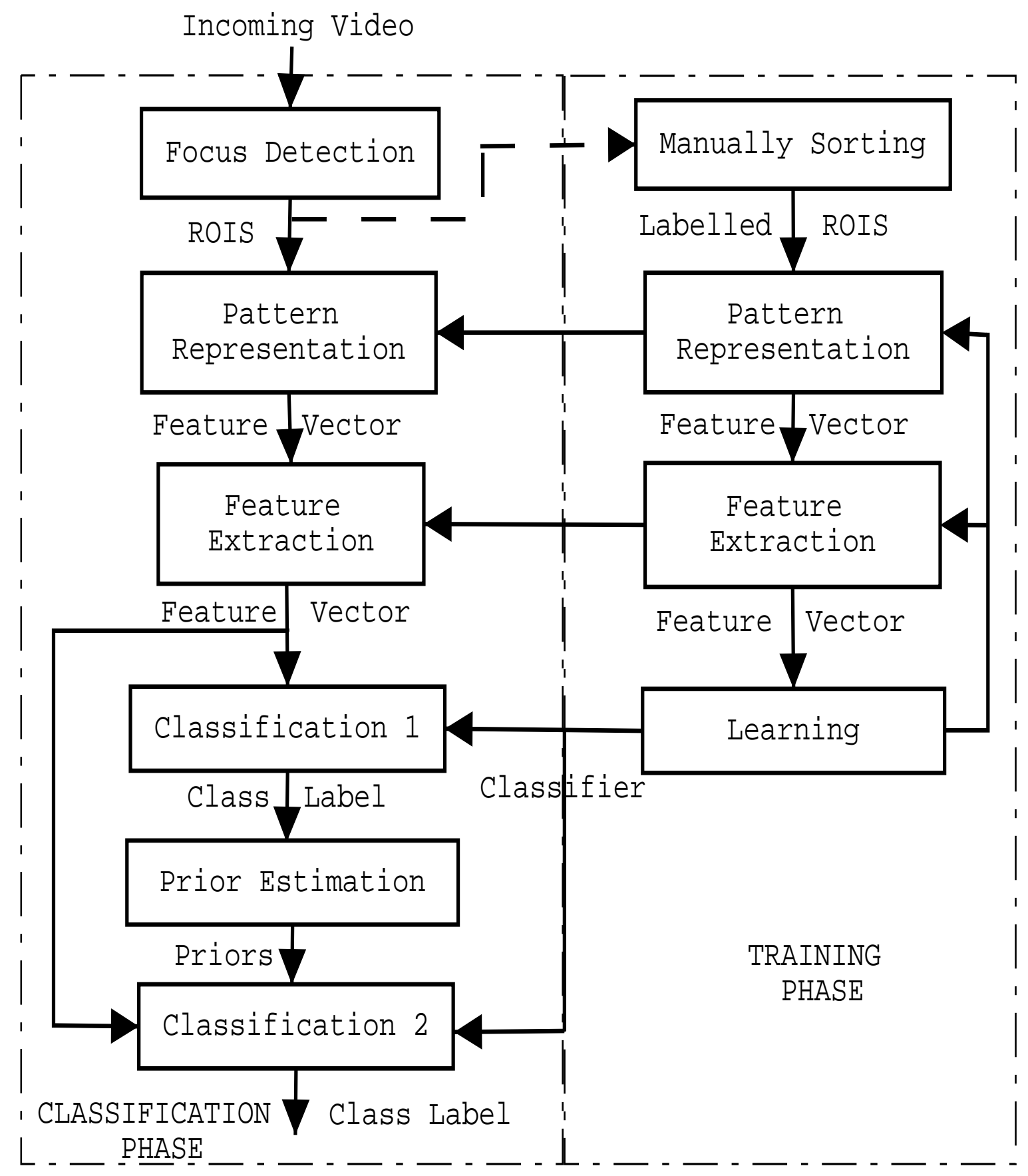

Figure 3-11: Schematic diagram of two pass classification system 
ure 3-12). For a taxon having a relative low abundance such as Chaetoceros chains, the abundance estimation from the single classifier is overestimated, as discussed before. More specifically, the synchronized abundance pattern between Chaetoceros chains and Chaetoceros socialis chains suggests that false alarms from Chaetoceros socialis chains dominate the abundance estimation of Chaetoceros chains. Two-pass classification makes abundance estimation of Chaetoceros chains a little closer to manually sorted results. Nonetheless, the correlation between Chaetoceros chains and Chaetoceros socialis chains is still marked in the two-pass classification system. In regions of relative low abundance, abundance estimation of the two-pass classification system matches very well with the manually sorted results. However, for rod-shaped diatom chains, two-pass classification system overestimates at relative high abundance. These overestimates come from the uneven probability of detection between rod-shaped diatom chains and the rest of the taxa.

\subsubsection{Discussion}

A two-pass classification system has been developed based on a neural network classifier. The two-pass classification works much better than the single classification in regions of relative low abundance. However, for species having relative high abundance and high probability of detection, this method tends to overestimate the abundance. Furthermore, for taxon having relative low abundance which has been coupled by other taxon, this method cannot fully decouple their dependancy.

The ideal conditions for the two-pass classification system is that each taxon in the classifier is independent of the other, and the probability of detection for each taxon is the same. In practice, two such conditions are not fully satisfied because two taxa may look like the other, or one taxon may be much easier to identify than all the other taxa. 

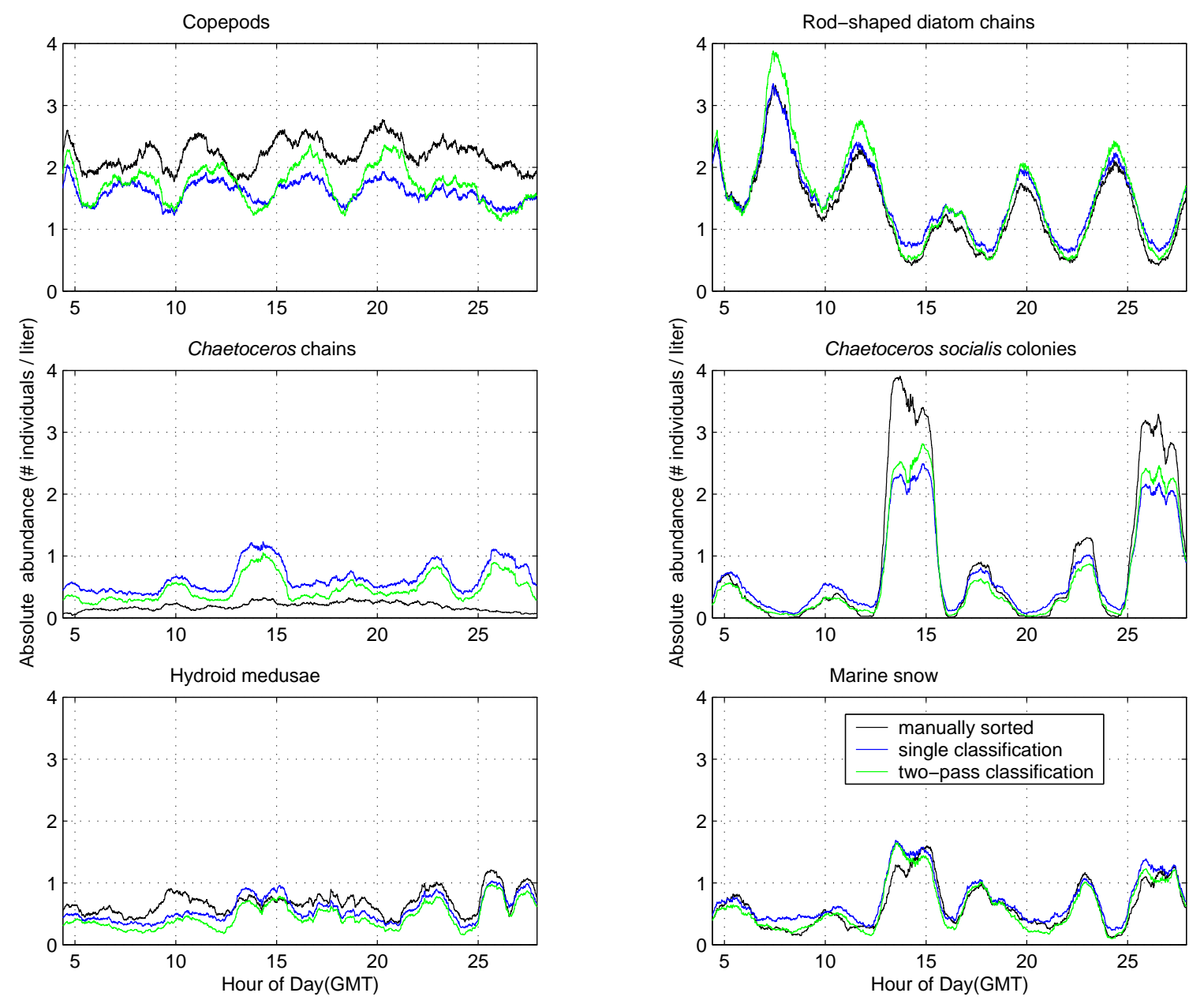

Figure 3-12: Comparison of two automatic classification systems with human expert classified results. Time series abundance plots along the tow path are shown for 6 dominant taxa. Data were first binned in 10 second time intervals, and a one-hour smoothing window was applied to the binned data. 


\subsection{Statistical correction method}

In the section, I try to correct bias from a single NN classification with a statistical correction method (SCM). As discussed in the last section, when the relative abundance is uneven and the classification system is not perfect, there is a high bias on taxon abundance estimation which has low relative abundance. In this section, I have proposed a method to correct this problem. Such a method is proposed by two independent research groups $[146,174]$.

\subsubsection{Method}

Before I start, I need to define confusion matrix. A confusion matrix is a way to quantify a classification system. It is an $n \times n$ matrix where $n$ is the number of taxon in the classifier. Elements $m_{i j}$ are the probabilities that a randomly selected sample of taxon $\omega_{j}$ will be classified to taxon $\omega_{i}$ by the classification system. Denote such a matrix as $M$.

Suppose the classification process can be characterized in the following mathematical model,

$$
\mathbf{x}_{\mathbf{C}}=M \mathbf{x}_{\mathbf{T}}
$$

where $\mathbf{x}_{\mathbf{C}}$ and $\mathbf{x}_{\mathbf{T}}$ are classified and true population proportion vectors. Assume $M$ is invertible, we can solve for $\mathbf{x}_{\mathbf{T}}$ using

$$
\mathbf{x}_{\mathbf{T}}=M^{-1} \mathbf{x}_{\mathbf{C}}
$$

Now suppose an estimate of confusion matrix $\hat{M}$ can be obtained by applying the classifier to a set of representative samples for each taxon or by the cross-validation method on training samples. Replacing $M$ by its estimated values $\hat{M}$ gives,

$$
\hat{\mathbf{x}}_{\mathbf{T}}=\hat{M}^{-1} \mathbf{x}_{\mathbf{C}}
$$

$\hat{\mathbf{x}}_{\mathbf{T}}$ is a maximum likelihood estimate of $\mathbf{x}_{\mathbf{T}}$, given the characteristics of the classifi- 
cation system described by the confusion matrix.

Negative abundance estimation may result from the above procedure, which indicates that there is an error in estimation of the confusion matrix $M$. However, such occurrences are very rare, given a fair wider smoothing window applied to the abundance estimation. One can choose an easy way to fix the negative abundance problem by setting negative abundance to zero and redistributing the net loss of abundance to the rest of the taxa in the classifier.

\subsubsection{Result}

Abundance estimations of six dominant taxa are compared among manually sorted, NN classifier and SCM with combined features of moment invariants, Fourier descriptors, pattern spectra and morphological measurements (Figure 3-13). Apparently, abundance estimation from SCM has lower bias compared to that of the automatic classification method. On the other hand, abundance estimation from SCM also yields a higher variance compared to that of the automatic classification method. This phenomenon is well-known, and is called the bias-variance dilemma or biasvariance trade-off, which states that classifiers with increased flexibility to adapt to the training data tend to have lower bias but higher variance. SCM obviously has much more flexibility than the uncorrected NN classifier.

Abundance estimation of rod-shaped diatom chains from SCM agrees very well with manually sorted estimation, although the abundance estimation from the NN classifier is already fairly good. On the other hand, abundance estimation of copepods from SCM has much larger error than that of the NN classifier. For Chaetoceros chains, SCM reduces the bias of abundance estimation; however, the artificial patchiness pattern still remains.

SCM yields lower bias and higher variance abundance estimation than the NN classifier. For applications investigating large scale abundance patterns or low frequency signals, SCM is a good method to use because some of the variance will go away with a wider smoothing window. On the other hand, for applications investigating small scale abundance patterns or high frequency signals, SCM should be 
avoided.
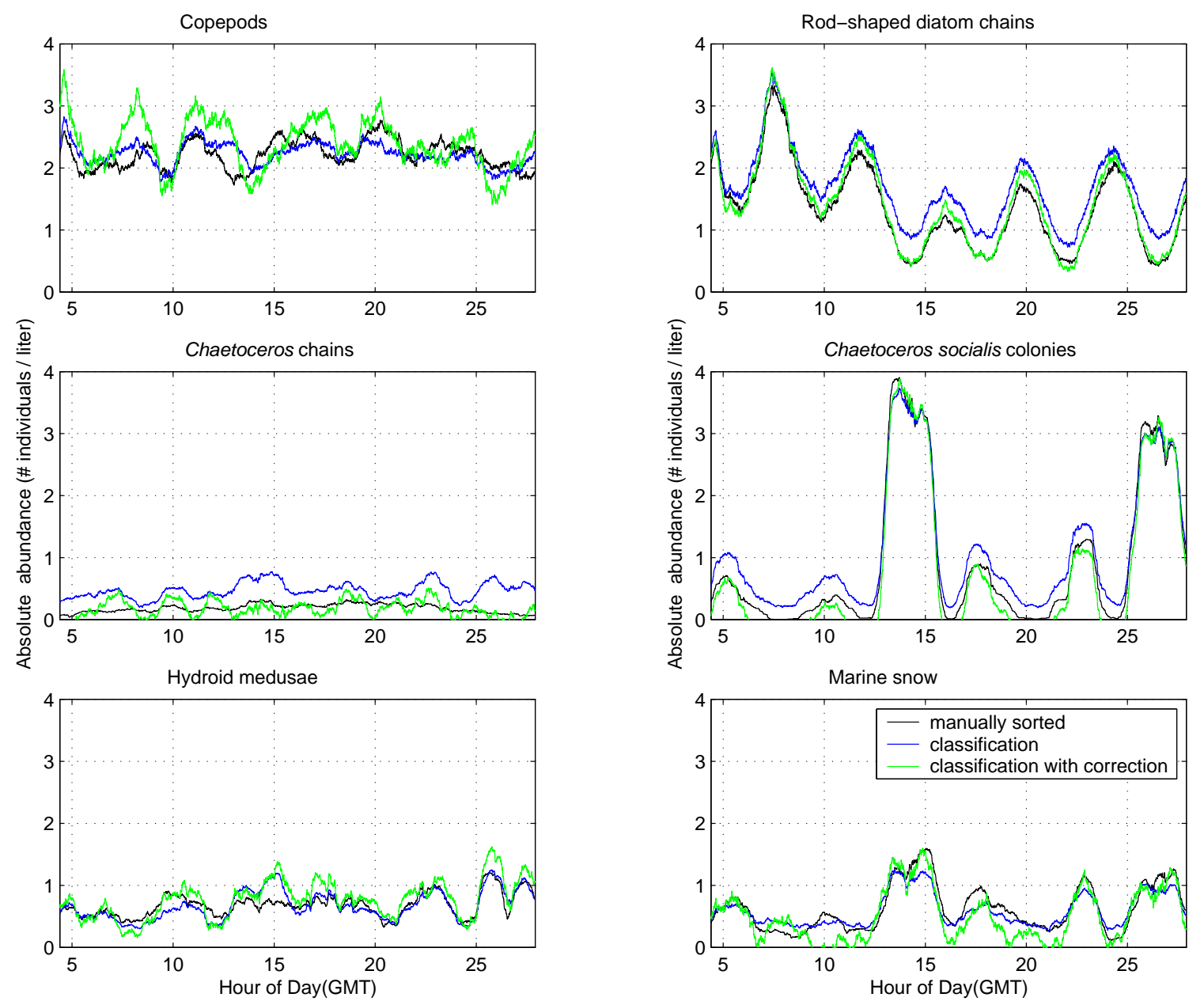

Figure 3-13: Comparison of automatic classification systems with/without statistical correction to human expert classified results. Time series abundance plots along the tow path are shown for 6 dominant taxa. Data were first binned in 10 second time intervals, and a one-hour smoothing window was applied to the binned data.

\subsection{Distance rejection metric for LVQ-NN classi- fier}

One of the problems of neural network classifiers (e.g. LVQ-NN classifier) is that it classifies all the novel samples into one of the taxa upon which it has been trained. However, many biological environments have an unbounded number of taxa. Es- 
pecially for exploration cruises, the taxa encountered during the cruise are hard to predict. Furthermore, in order for an LVQ-NN classifier to reliably recognize new samples in each taxon, a certain number of samples in each taxon is required. In many applications, there are not enough training samples to train an LVQ-NN classifier for some taxa. In such cases, it is essential for a classifier to be able to reject the novel samples as "unknown" instead of classifying incorrectly into one of the taxa in which the classifier has been trained. In this section, I have developed a rejection metric based on distance map between neurons and test samples.

\subsubsection{Distance rejection system}

The schematic diagram is shown in Figure 3-14. The difference between an LVQNN classification system with and without distance rejection metric is that, after a normal LVQ-NN classifier has been trained, all the training samples are classified once again by the freshly trained classifier; the distances between each sample and the nearest neuron are recorded for every sample which is correctly classified by the LVQ-NN classifier. Mean and standard deviation of the distances are calculated for each class in the classifier and a distance outlier threshold has been computed from the mean and standard deviation of the distance. That is to say, after training, besides a normal LVQ-NN classifier, a set of distance outlier thresholds have been obtained for each class in the classifier. During the classification process, when each sample is classified by a normal LVQ-NN classifier, the distance between the sample and the nearest neuron has been compared against the distance threshold of the class upon which the normal LVQ-NN classifier is going to predict. If the distance is below the threshold, the classifier predicts the label of the sample that is the same as that neuron. Otherwise, the classifier predicts the label of the sample as "unknown".

\subsubsection{Result and discussion}

In order to test the distance rejection metric, a random set of samples with 200 per taxon for training and another 200 per taxon were drawn from the whole data set. In 


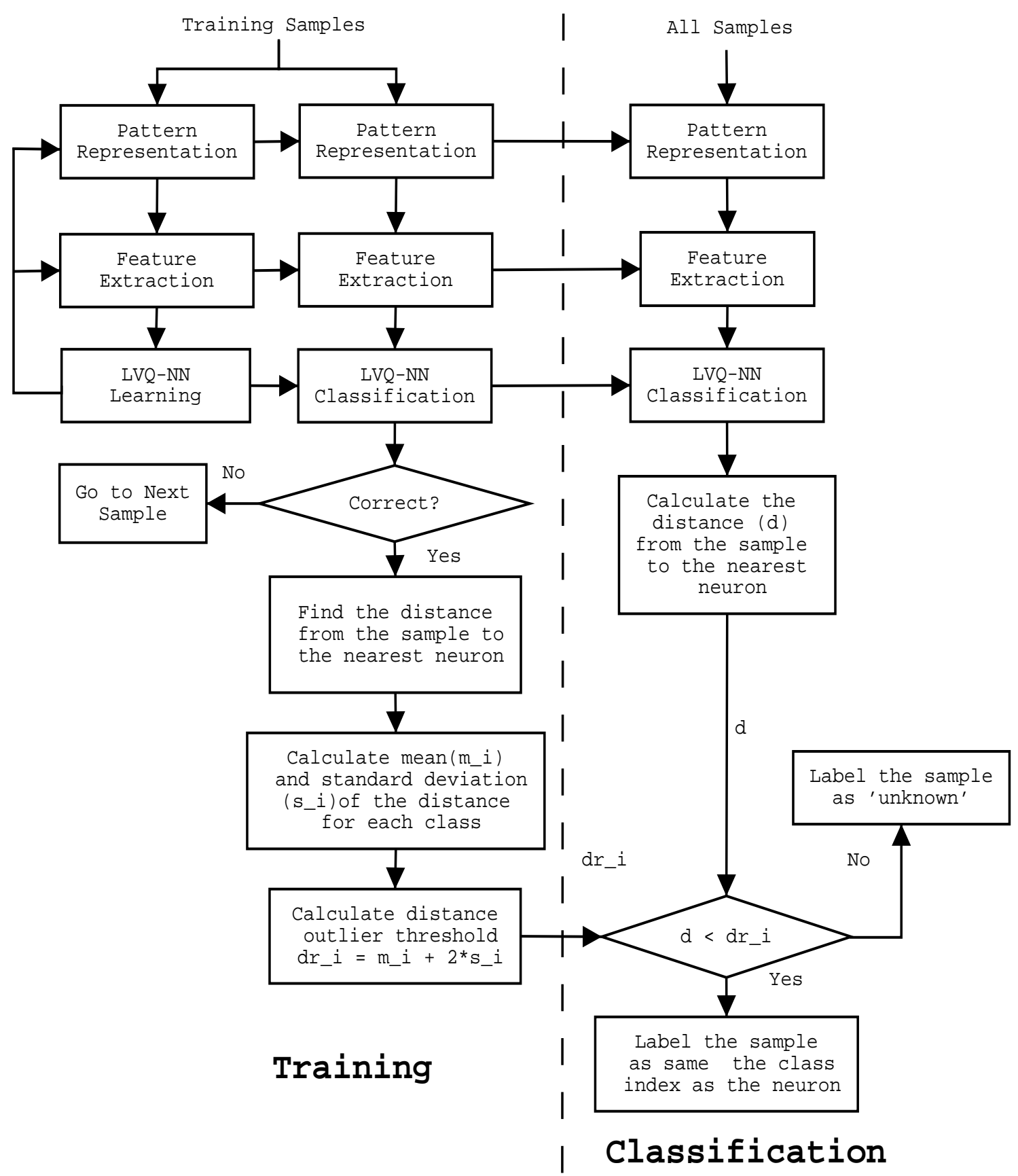

Figure 3-14: Schematic diagram of distance rejection classification system 
order to avoid dealing overlapping issues between copepod and the "other" category, only 6 major taxa were used in this study. The LVQ-NN classifier was trained from 5 out of 6 major taxa. The classifier was used to classify all the test samples of 6 taxa. The results were summarized in Tables 3.1 to 3.6. To read these results, one should focus on the last row and last column of these matrices. The last row tells us how many test samples were classified as "unknown" (being rejected), and the last column tells us how the classifier classified the novel samples.

From Tables 3.1 to 3.6, we can calculate that the rejection ratio of "known" classes was about 5\%, which was acceptable. However, the rejection ratio of novel classes was less than $10 \%$, which was way too low. The low rejection ratio of novel classes suggested that most of the novel samples did not look "novel" in the feature space. In other words, the novel samples and "known" samples looked alike in the feature space.

The failure of distance rejection metric implies that the combined shape-based features were not good enough to separate different taxa apart in this application. This study leads me to look for other pattern representation methods in the next chapter.

Table 3.1: Confusion matrix of an LVQ-NN classifier with distance rejection metric using hold-out method. The classifier was trained without marine snow. Column and row heading are coded as: C1, copepod; C2, rod-shaped diatom chains; C3, Chaetoceros chains; C4, Chaetoceros socialis colonies; C5, hydroid medusae; C6, marine snow; C6*, "unknown".

\begin{tabular}{||l|l|l|l|l|l|r||}
\hline \hline & C1 & C2 & C3 & C4 & C5 & C6 \\
\hline C1 & 150 & 12 & 16 & 10 & 11 & 27 \\
\hline C2 & 11 & 173 & 1 & 1 & 7 & 4 \\
\hline C3 & 6 & 3 & 135 & 13 & 39 & 61 \\
\hline C4 & 12 & 4 & 22 & 148 & 10 & 38 \\
\hline C5 & 14 & 5 & 18 & 11 & 115 & 63 \\
\hline C6 $^{*}$ & 7 & 3 & 8 & 17 & 18 & 7 \\
\hline \hline
\end{tabular}


Table 3.2: Confusion matrix of an LVQ-NN classifier with distance rejection metric using hold-out method. The classifier was trained without hydroid medusae. Column and row heading are coded as: C1, copepod; C2, rod-shaped diatom chains; C3, Chaetoceros chains; C4, Chaetoceros socialis colonies; C5, marine snow; C6, hydroid medusae; $6^{*}$, "unknown".

\begin{tabular}{||l|l|l|l|l|l|r||}
\hline \hline & C1 & C2 & C3 & C4 & C5 & C6 \\
\hline C1 & 133 & 14 & 11 & 6 & 13 & 11 \\
\hline C2 & 18 & 166 & 4 & 6 & 0 & 11 \\
\hline C3 & 7 & 3 & 131 & 18 & 32 & 40 \\
\hline C4 & 11 & 2 & 12 & 136 & 21 & 16 \\
\hline C5 & 18 & 7 & 30 & 23 & 125 & 92 \\
\hline C6 & 13 & 8 & 12 & 11 & 9 & 30 \\
\hline \hline
\end{tabular}

Table 3.3: Confusion matrix of an LVQ-NN classifier with distance rejection metric using hold-out method. The classifier was trained without Chaetoceros socialos colonies. Column and row heading are coded as: C1, copepod; C2, rod-shaped diatom chains; C3, Chaetoceros chains; C4, hydroid medusae; C5, marine snow; C6, Chaetoceros socialis colonies; C6*, "unknown".

\begin{tabular}{||l|l|l|l|l|l|r||}
\hline \hline & C1 & C2 & C3 & C4 & C5 & C6 \\
\hline C1 & 139 & 11 & 15 & 8 & 13 & 9 \\
\hline C2 & 13 & 172 & 5 & 8 & 5 & 6 \\
\hline C3 & 10 & 2 & 118 & 20 & 38 & 68 \\
\hline C4 & 9 & 5 & 23 & 122 & 35 & 18 \\
\hline C5 & 19 & 3 & 26 & 24 & 106 & 85 \\
\hline C6 $^{*}$ & 10 & 7 & 13 & 18 & 3 & 14 \\
\hline \hline
\end{tabular}

Table 3.4: Confusion matrix of an LVQ-NN classifier with distance rejection metric using hold-out method. The classifier was trained without Chaetoceros chains. Column and row heading are coded as: C1, copepod; C2, rod-shaped diatom chains; C3, Chaetoceros socialos colonies; C4, hydroid medusae; C5, marine snow; C6, Chaetoceros chains; C6*, "unknown".

\begin{tabular}{||l|l|l|l|l|l|r||}
\hline \hline & C1 & C2 & C3 & C4 & C5 & C6 \\
\hline C1 & 144 & 10 & 3 & 5 & 17 & 25 \\
\hline C2 & 5 & 169 & 3 & 5 & 5 & 0 \\
\hline C3 & 10 & 2 & 153 & 14 & 42 & 28 \\
\hline C4 & 9 & 2 & 9 & 122 & 37 & 57 \\
\hline C5 & 20 & 8 & 24 & 39 & 94 & 73 \\
\hline C6 & 12 & 9 & 8 & 15 & 5 & 17 \\
\hline \hline
\end{tabular}


Table 3.5: Confusion matrix of an LVQ-NN classifier with distance rejection metric using hold-out method. The classifier was trained without rod-shaped diatom chains. Column and row heading are coded as: C1, copepod; C2, Chaetoceros chains; C3, Chaetoceros socialis colonies; C4, hydroid medusae; C5, marine snow; C6, rod-shaped diatom chains; $\mathrm{C} 6 *$, "unknown".

\begin{tabular}{||l|l|l|l|l|l|r||}
\hline \hline & C1 & C2 & C3 & C4 & C5 & C6 \\
\hline C1 & 149 & 11 & 4 & 16 & 12 & 120 \\
\hline C2 & 14 & 118 & 10 & 20 & 23 & 10 \\
\hline C3 & 9 & 9 & 145 & 4 & 27 & 7 \\
\hline C4 & 3 & 23 & 8 & 113 & 38 & 43 \\
\hline C5 & 16 & 27 & 23 & 30 & 96 & 8 \\
\hline C6 & 9 & 12 & 10 & 17 & 4 & 12 \\
\hline \hline
\end{tabular}

Table 3.6: Confusion matrix of an LVQ-NN classifier with distance rejection metric using hold-out method. The classifier was trained without copepod. Column and row heading are coded as: C1, rod-shaped diatom chains; C2, Chaetoceros chains; C3, Chaetoceros socialis colonies; C4, hydroid medusae; C5, marine snow; C6, copepod; C6*, "unknown".

\begin{tabular}{||l|l|l|l|l|l|l||}
\hline \hline & C1 & C2 & C3 & C4 & C5 & C6 \\
\hline C1 & 176 & 4 & 3 & 2 & 5 & 45 \\
\hline C2 & 6 & 118 & 17 & 25 & 25 & 55 \\
\hline C3 & 2 & 16 & 136 & 5 & 32 & 20 \\
\hline C4 & 4 & 17 & 12 & 122 & 35 & 18 \\
\hline C5 & 8 & 33 & 23 & 27 & 99 & 38 \\
\hline C6 & 4 & 12 & 9 & 19 & 4 & 24 \\
\hline \hline
\end{tabular}




\subsection{Conclusion}

In this chapter, a classic learning vector quantization neural network classifier with combined shape-based features was assessed. Multiple factors such as classifier complexity, number of training samples, quality of training samples, feature length, classifier stability, and presentation order of training samples were investigated. This study showed that previous reported accuracy was optimistically biased. A two-pass classification system and a statistical correction method were developed on this classifier. A distance rejection metric was also developed on the LVQ-NN classifier. The limited improvement on various methods based on the LVQ-NN classifier suggested that the shape-based features were not good enough to classify field-collected plankton images. The study leads me to look for texture-based features in the next chapter. 


\section{Chapter 4}

\section{Pattern Representation/Feature}

\section{Measurement}

This chapter covers the various pattern representation methods used to obtain features from pattern images. Although pattern recognition has been well studied, pattern does not have a well-accepted definition. According to Watanabe [165], a pattern is opposite to a chaos; it is an entity, vaguely defined, that could be given a name. For example, a pattern could be a human face image or an acoustic signal. In this thesis, I consider a pattern as a view-based 2-D image of a 3-D object. In pattern recognition/classification, a set of measurements which describes a pattern is of special interest. These measurements are called features, and the step that calculates features from pattern is called pattern representation or feature measurement ${ }^{1}$. Three important cues to recognize an image object are shape, texture, and color. Similarly, pattern representation methods can be grouped into shape-based, texture-based, and color-based method. In this chapter, shape-based and texture-based feature methods are discussed and compared using a random subset of the data set described in Chapter 1 . The images are in-focus regions-of-interest (ROIs) extracted from the full 2-D grayscale images digitized from the VPR's analog video (see Chapter 2).

This chapter is organized as follows. In Section 4.1, I review the pattern represen-

\footnotetext{
${ }^{1} \mathrm{~A}$ number of authors use the term feature extraction to cover all the processes from raw data to final feature set, which includes pattern representation and feature selection/extraction in our terminology.
} 
tation in the literature, and group them into 3 major categories, namely shape-based, texture-based, and other methods. In Section 4.2, a selected representative methods from each group and their feature extraction and classification method are described. In Section 4.3, I compare selected pattern representation methods and different feature extraction for each method.

\subsection{Pattern representation methods}

\subsubsection{Shape-based methods}

Shape-based features are features calculated primarily from the shape of objects. Classic examples of shape-based features are moment invariants and Fourier desciptors.

\section{Moment Invariants}

Moment invariants were first introduced by $\mathrm{Hu}[72]$ to classify planar objects. They are one of the most extensively studied invariant features $[7,101,131]$. These orthogonal moment invariants are invariant to rotation, scale and translation (RST). For non-negative integers $p, q$, the $(p+q)^{t h}$ order moments of a pattern $f(x, y)$ are given as,

$$
m_{p, q}=\int_{-\infty}^{\infty} \int_{-\infty}^{\infty} x^{p} y^{q} f(x, y) d x d y
$$

Its discrete form is

$$
m_{p, q}=\sum_{x=1}^{M} \sum_{y=1}^{N} f(x, y) x^{p} y^{q},
$$

where the image is size of $M \times N$. The translation-invariant central moments of order $(p+q)$ are obtained by placing the origin at the center of gravity

$$
\mu_{p, q}=\sum_{x=1}^{M} \sum_{y=1}^{N} f(x, y)(x-\bar{x})^{p}(y-\bar{y})^{q}
$$


where $\bar{x}=m_{10} / m_{00}, \bar{y}=m_{01} / m_{00}$.

$\mathrm{Hu}$ showed that

$$
\nu_{p q}=\frac{\mu_{p q}}{\mu^{(1+(p+q) / 2)}}, \quad p+q \geq 2
$$

were scale-invariant, where $\mu=\mu_{00}=m_{00}$. From combination of $\nu_{p q}$ 's, $\mathrm{Hu}[72]$ derived seven moment invariants which were RST invariant.

\section{Zernike moments}

The Zernike polynomials were first introduced in 1934 [176]. For a set of complex Zernike polynomials $V_{n m}(x, y)$, which form a complete orthogonal basis defined within the unit circle, Zernike moments of an image function $f(x, y)$ are the projection of the function onto Zernike polynomials

$$
A_{n m}=\frac{n+1}{\pi} \iint_{x^{2}+y^{2} \leq 1} f(x, y)\left[V_{n m}(x, y)\right]^{*} d x d y .
$$

Here $n \geq 0, \quad|m| \leq n, \quad n-|m|$ is even, and the symbol $*$ denotes the complex conjugate operator. A set of complex orthogonal polynomials $V_{n m}(x, y)$ are defined as

$$
V_{n m}(x, y)=R_{n m}(x, y) e^{j m \arctan (y / x)}
$$

where $j=\sqrt{-1}, n \geq 0,|m| \leq n, n-|m|$ is even, and

$$
R_{m n}(x, y)=\sum_{s=0}^{(n-|m|) / 2} \frac{(-1)^{s}\left(x^{2}+y^{2}\right)^{(n / 2)-s}(n-s) !}{s !\left(\frac{(n+|m|)}{2}-s\right) !\left(\frac{(n-|m|)}{2}-s\right) !}
$$

For a digital image, the Zernike moment of order $n$ and repetition $m$ is given by

$$
A_{n m}=\frac{n+1}{\pi} \sum \sum_{x^{2}+y^{2} \leq 1} \quad f(x, y)\left[V_{n m}(x, y)\right]^{*}
$$

Here the symbol $*$ denotes the complex conjugate operator.

Zernike polynomials are orthogonal and rotation invariant. Zernike moments have nice properties in terms of noise sensitivity, information redundancy and reconstruc- 
tion capability [151]. The amplitudes of the Zernike moments were used as features for character recognition [7, 90,91], texture classification [163], and invariant image watermark [93].

\section{Fourier-Mellin Transform}

Let $f(r, \theta)$ be an intensity function of a two-dimensional image expressed in the polar coordinates. By first applying the circular Fourier transform and then applying Mellin transform to this function, we have the following transform as a function of $l$ and $w$

$$
z(l, w)=\frac{1}{2 \pi} \int_{0}^{\infty} \int_{0}^{2 \pi} f(r, \theta) e^{-i l \theta} r^{i w-1} d \theta d r
$$

This is the so called Circular-Fourier Radial-Mellin Transform, or simply the FourierMellin Transform. The modulus of $z(l, w)$ is invariant under both rotation and scaling. The coefficients $z(l, w)$ are often referred to as Fourier-Mellin descriptors. Their magnitudes are often used as invariant features under the two-dimensional rotation and scaling $[36,86,141,142,143]$. In order to achieve translation invariance, one can shift the origin of polar coordinates at the center of gravity. Casasent and Psaltis $[18,19]$ took the following alternative approach.

1. Calculate the power spectrum of the Fourier transform of the two-dimensional input.

2. Convert the power spectrum to polar coordinates.

3. Perform a polar-log mapping.

4. Calculate another two-dimensional Fourier transform power spectrum.

The final power spectrum is RST invariant. Li [101] has identified that the normalized Fourier-Mellin descriptors are linear combinations of some normalized geometric moments. 


\section{Fourier Descriptors}

Fourier descriptors (FD) are well studied invariant features used to describe a contour of an object. Depending on what functions are used to describe a contour, FDs can be grouped into 3 major categories, namely tangent-angle FDs proposed by Zahn and Roskies [175], complex FDs first used by Granlund [57], and elliptic FDs by Kuhl and Giardina [94]. FDs are the Fourier coefficients when the contour function is approximated by Fourier series. More research effort has been devoted to the shape classification by FDs [123, 102, 89]. For the most common used contour complex function, each point on the contour can be represented by its complex coordinates, $z(l)=x(l)+j y(l)$. As a point moves along the contour in the counterclockwise direction, it generates a complex function $z(l)$. Suppose we normalize the perimeter of the contour to $2 \pi$, then the function $z(l)$ is periodic with the period of $2 \pi$. Such a function can be approximated by a Fourier series,

$$
z(l)=\sum_{k=-\infty}^{\infty} c_{k} e^{j k l} .
$$

The coefficients can be calculated from the following integral,

$$
c_{k}=\frac{1}{2 \pi} \int_{-\pi}^{\pi} z(l) e^{-j k l} d l
$$

The normalized amplitude spectrum $\left\{\left|c_{n}\right| /\left|c_{1}\right|\right\} \quad(n \neq 0)$ is invariant to RST and reflection.

\section{Curvature and Shape Spectrum}

Gaussian curvature (curvature for short) is an intrinsic property of a 2-dimensional surface. It is independent of the coordinate system. In two dimensions, the extrinsic curvature is defined as

$$
\kappa \equiv \frac{d \phi}{d s}
$$


which can be calculated as

$$
\kappa=\frac{x^{\prime} y^{\prime \prime}-y^{\prime} x^{\prime \prime}}{\left(x^{\prime 2}+y^{\prime 2}\right)^{3 / 2}}
$$

The Gaussian curvature and mean curvature are defined from the two principal curvatures. The Gaussian curvature is defined as the product of the two principal curvatures, while mean curvature is the mean of the two principal curvatures. Gaussian curvature is used as a shape descriptor to describe $3-\mathrm{D}$ objects $[132,54]$. Dorai and

Jain $[41,40]$ proposed to use the shape index to describe $3-\mathrm{D}$ free-form objects. The shape index is defined as

$$
S_{I}(p)=\frac{1}{2}-\frac{1}{\pi} \tan ^{-1} \frac{\kappa_{1}(p)+\kappa_{2}(p)}{\kappa_{1}(p)-\kappa_{2}(p)}
$$

where $\kappa_{1}$ and $\kappa_{2}$ are the principal curvatures of the surface. The histogram of the shape index is called the shape spectrum. Nastar[119] applied the shape spectrum to real-time face recognition.

\subsubsection{Texture-based methods}

Image texture is a function of spatial variations in pixel intensities. It is difficult to give texture a formal definition [156]. On the other hand, texture is the most important visual cue in identifying different types of homogeneous images via their texture properties. Most natural surfaces reveal unique texture. Texture analysis has applications in texture classification, segmentation and synthesis.

\section{Autocorrelation and Power Spectrum}

In a simple model, texture is considered as a repetitive placement of texture elements (primitive or texton) in the image. The autocorrelation method [127] is based on finding linear spatial relationships between primitives. Given a gray-scale image $f(x, y)$, the autocorrelation function is defined as

$$
C_{p, q}=\frac{\sum_{x=1}^{M} \sum_{y=1}^{N} f(x, y) f(x+p, y+q)}{\sum_{x=1}^{M} \sum_{y=1}^{N} f^{2}(x, y)} .
$$


If the primitives are large, the function decreases slowly with increasing distance, whereas it decreases rapidly if texture consists of small primitives. The power spectrum is highly related to autocorrelation function. The discrete Fourier transform of an image $f\left(n_{1}, n_{2}\right)$ is defined by

$$
F\left(k_{1}, k_{2}\right)=\left\{\begin{array}{l}
\sum_{n_{1}=0}^{N_{1}-1} \sum_{n_{2}=0}^{N_{2}-1} f\left(n_{1}, n_{2}\right) e^{-j\left(2 \pi / N_{1}\right) k_{1} n_{1}} e^{-j\left(2 \pi / N_{2}\right) k_{2} n_{2}} \\
\quad 0 \leq k_{1} \leq N_{1}-1, \quad 0 \leq k_{2} \leq N_{2}-1 \\
0, \quad \text { otherwise. }
\end{array}\right.
$$

The power spectrum is defined as

$$
P\left(k_{1}, k_{2}\right)=F\left(k_{1}, k_{2}\right) \cdot F\left(k_{1}, k_{2}\right)^{*}
$$

\section{Co-occurrence Matrices}

Spatial gray level co-occurrence matrices estimate second-order statistics from the images. Julesz [85] did pioneering work on texture analysis with first-order and second-order statistics. The co-occurrence matrices method was first proposed by Haralick [59] as a texture feature and it has been widely used thereafter. It is based on estimation of the joint probability distribution of pixels with gray level $i$ and $j$, a spatial distance $d$, and angle $\theta$ in an image. If the texture is coarse and distance $d$ is small compared to the size of texture primitive, the pairs of points should have similar gray levels. Conversely, for a fine texture, if distance $d$ is comparable to the texture size, the gray levels of point pairs should be quite different. The value in the co-occurrence matrix should be spread out relatively uniformly. Hence, a good way to analyze texture coarseness would be, for various values of distance $d$, some measurement of the scatter of the co-occurrence matrix around the main diagonal. Similarly, if the texture has some direction (i.e., coarser in one direction than another), the degree of spread along the main diagonal in the co-occurrence matrix should vary with the direction $\theta$. Therefore texture directionality can be analyzed by comparing the spread of co-occurrence matrices constructed at various distances 
d. From co-occurrence matrices a variety of such statistical measurements may be extracted.

\section{Edge Frequency}

The edge frequency method [153] computes the gradient difference between a pixel $f(x, y)$ and its neighbors at a distance $d$. For computational efficiency, only four directions are used. For a given value of distance $d$, the gradient differences $g(d)$ are summed up over the whole image. In this study I use a slightly different formula; I keep the spectra from four directions separated. For example, $g(d)$ can be calculated $\mathrm{as}^{2}$

$$
\begin{aligned}
g_{0}(d) & =\sum_{x=1}^{M} \sum_{y=1}^{N}|f(x, y)-f(x+d, y)|, \\
g_{45}(d) & =\sum_{x=1}^{M} \sum_{y=1}^{N}|f(x, y)-f(x+d, y+d)|, \\
g_{90}(d) & =\sum_{x=1}^{M} \sum_{y=1}^{N}|f(x, y)-f(x, y+d)|, \\
g_{135}(d) & =\sum_{x=1}^{M} \sum_{y=1}^{N}|f(x-d, y)-f(x, y+d)|,
\end{aligned}
$$

For different values $d$, a spectrum is obtained. In two direction formulation, spectra from horizontal and vertical are combined, so are the two diagonal directions. The micro edges are detected by small distance operators, while macro edges are captured by long distance operators.

\section{Law's Energy Filter}

Law $[95,127]$ proposed nine $3 \times 3$ pixel impulse response masks to accentuate microstructure. All masks are convolved with the input image. Let $f(x, y)$ be the brightness of an image, and $h_{i}(x, y)$ the $i$ th mask, the $i$ th micro-structure array is $g(x, y)=$

\footnotetext{
${ }^{2}$ Single forward direction is used since the formula is symmetric with $d$, and bidirectional formulation yields twice the value of unidirectional formulation
} 
$f(x, y) \star h_{i}(x, y)$. The energy is measured by forming a moving window standard deviation on these micro-structure arrays.

\section{Primitive Length/Run Length}

A primitive is the set of the maximum number of pixels in the same direction that have the same gray level. For a coarse texture, a large number of neighboring pixels would be on the same gray level, while a small number of neighboring pixels would be on the same gray level for a fine texture. Based on above observation, Galloway [52] proposed to use a gray level run-length matrix for texture features. Let $B(i, r)$ be the number of primitives in all directions with length $r$ and gray level $i, L$ be the number of image gray level, $N_{r}$ be the maximum length of the primitive, then the total number of primitives is

$$
K=\sum_{i=1}^{L} \sum_{r=1}^{N_{r}} B(i, r) .
$$

Based on $B(i, r)$ and $K$, a set of statistics is calculated, which includes short primitive emphasis, long primitive emphasis, gray-level uniformity, primitive length uniformity, primitive percentage [52], low gray-level run emphasis, high gray-level run emphasis [22], short run low gray-level emphasis, short run high gray-level emphasis, long run low gray-level emphasis, and long run high gray-level emphasis [29].

\section{Binary Stack Method}

Chen et al. [21] introduced binary stacks for texture analysis. For a total of $L$ gray levels, $L$ binary images are generated by thresholding the original image at each gray level. The resulting stack of binary images is analyzed by grouping all 1- and 0-valued pixels into connected regions. For each connected region, irregularity or circularity is calculated and weighted based on the total size of connected components. 


\section{Logical Operator}

Manian et al. [109] presented a new algorithm for texture classification based on logical operators. Operators constructed from logical building blocks are convolved with texture images. An optimal set of six operators are used and convolved with images. The responses are then converted to standard deviation matrices computed over a moving window. Zonal sampling features are computed from these matrices.

\section{Texture Spectrum}

He and Wang [65] proposed to use texture spectrum for extracting texture features. If an image is considered to be composed of small texture units, the frequency distribution of these texture units is a texture spectrum. The features extracted include black-white symmetry, geometric symmetry, degree of direction, orientation features and central symmetry.

\section{Pattern Spectrum}

Mathematical morphology has its roots in the pioneering work of Matheron [112] and Serra [140]. Matheron used a series of openings and closings to obtain probabilistic size distributions of Euclidean-space sets (continuous binary images). These distributions can be viewed as a concept of a shape-size descriptor, which is later called pattern spectrum. This idea then was extended to grayscale images and studied by different authors $[111,56]$. Unfortunately, the normal methods involve a series of structural openings and closings to the input image, which is computationally expensive. Recently, fast approximation algorithms have become available to estimate pattern spectra with very limited structural elements [162, 114], which makes pattern spectra computation possible in real-time applications. Tang et al. [150] used granulometry as part of features to classify plankton images. 


\subsubsection{Other Methods}

\section{Gabor Filter}

The Gabor function was first introduced in 1-D [51]. It was later extended to 2-D Gabor filters [30, 157, 125, 78]. Gabor filters are band-pass filters which have both orientation and frequency selective properties. Daugman [30] suggested to use Gabor filters in the modeling of visual cortical receptive fields of mammals. Turner [157], Clark and Bovik [23] proposed to use Gabor filters in texture analysis. A 2-D Gabor function consists of a sinusoidal plane wave with a certain frequency and orientation modulated by a Gaussian envelope. It has the following form,

$$
G(x, y)=\exp \left(-\frac{1}{2}\left[\frac{x^{2}}{\sigma_{x}^{2}}+\frac{y^{2}}{\sigma_{y}^{2}}\right]\right) \cos (2 \pi f x+\phi),
$$

where $f$ and $\phi$ are the frequency and phase of the sinusoidal plane wave. The $\sigma_{x}$ and $\sigma_{y}$ specify the widths of the Gaussian envelope along $x$ and $y$ directions, respectively. The selection of the values of $\sigma_{x}$ and $\sigma_{y}$ is based on the trade-off between robustness to noise and the loss of image details. If these values are too large, the filter is more robust to noise, but is more likely to smooth out the image details. On the other hand, if these values are too small, the filter is not effective enough to remove noise. Jain [78] successfully applied the Gabor filter to extract features from fingerprint images.

\section{Wavelet Transform}

Wavelets are a type of multiresolution and multi-scale functions that allow hierar-

chical decomposition of a signal. When applied at different scales, wavelets encode information about an image from the coarse approximation all the way down to the fine details. It has received wide attention on texture classification and image segmentations $[20,158,128]$. 


\section{Scale Invariant Feature Transform}

Lowe and Brown $[104,14]$ used scale-invariant feature transform to identify 3-D objects. The scale-invariant features are identified by a staged filtering approach. The first stage identifies key location, scale, and orientation for each key. The key locations are the maxima or minima of a difference-of Gaussian (DoG) function applied in scale space. The second stage uses a feature vector that describes the local image region of each key location. The feature vector is the orientation measurement relative to that of the key, by subtracting the key's orientation. The eight orientation planes are evaluated at different locations and spatial scales.

\section{Component-based approach}

Mohan et al. [118] used a component-based system to detect people in clutter scenes. The system is structured with four distinct detectors to be trained to find four components of a human body: the head, legs, left arm, and right arm. Haar wavelets of two different scales are used to generate a multi-scale representation of the images. The wavelets are applied to the images with $75 \%$ overlapping windows. Heisele et al. [66] used the same idea as a face detector, which used fourteen components to describe a face.

\section{Deformable template models}

The deformable models have wide applications in pattern recognition and computer vision, including image/video database retrieval [11], object recognition and identification [16, 80], image segmentation [155], and object tracking [53, 100].

In the section, deformable template models are surveyed, which are based on Jain et al. [79] There are two classes of deformable models. The free-form models (active contour models) are able to model any shape using general constraints (such as continuity, smoothness). On the other hand, the parametric deformable models are able to model one kind of shape and its variation.

The snake model [87] is one of the most successful free-form deformable models. 
In the snake model, a contour, called a 'snake', is continuously updated based on the following three forces or energies: 1) an internal contour force which controls smoothness of the contour, 2) an image force which attracts the contour to the desired

shape, and 3) an external constraint force. The internal contour force and the image force have opposite direction. The contour actively adjusts its position and shape when these two forces interact with each other. The contour stops to involve when its energy reaches a local minimum:

$$
\mathcal{E}_{\text {snake }}=\int_{0}^{1}\left(\mathcal{E}_{\text {intern }}(p(s))+\mathcal{E}_{\text {image }}(p(s))+\mathcal{E}_{\text {extern }}(p(s))\right) d s,
$$

where $s$ is the parameterization of the contour, and $p(s)$ is a point on the contour.

Parametric deformable models are more useful when some prior information of the geometrical shape is available, which can be encoded by a small number of parameters. There are two ways to parameterize the shape of an object and its variations. The analytical deformable templates are decomposed by a set of analytical curves. Each curve can be represented by a few parameters. The geometrical shape and its variations of the object are controlled by different values of the parameters. The prototype-based deformable templates are represented by a 'prototype' template which characterizes the 'most likely', or 'average' shape of a group of objects. Each instance of the shape class and its 'prototype' are linked through paremetric mapping. Variations in the shape are determined by the parameter values which define the mapping.

\subsection{Feature extraction and classification}

Moment invariants (MI), Fourier descriptors (FD), curvature and shape spectrum (CSS), co-occurrence matrices (COM), edge frequency (EF), run length (RL), pattern spectrum (PS), wavelet transform (WT), and morphological measurement (MM) methods are compared using a subset of plankton images in this chapter.

For moment invariants and Fourier descriptors features, the images are first seg- 
mented using Otsu's global threshold selection method [121]. Moment invariants order up to 3 [72] through 7 [101] from binary images are computed, which correspond to the feature length of $7,12,18,24$, and 33 respectively.

For radial Fourier descriptors (RFD) and Complex Fourier descriptors (CFD), a contour is calculated from the largest blob in each image. The contour is first interpolated into 256 pixels using linear interpolation. Then the contour is expressed by radial function from the centroids of the object or by complex coordinates of the objects. Discrete Fourier transform is taken from these functions. The first 64 Fourier coefficients (RFD) are normalized by the first element or the 128 element of Fourier power spectrum (CFD) is normalized by second element as a feature vector.

For Shape spectrum features, the image is first smoothed by a Gaussian kernel of size $9 \times 9$ and width $\sigma=9 / 4$. Principal curvatures are computed from smoothed images. Shape spectrum is calculated as suggested by Dorai and Jain [41, 40]. The histogram of shape spectrum with 128 bins is used as features. The high peaks at .25, .5 and .75 are suppressed by replacing them with the average of their two neighbors.

For the co-occurrence matrices method, each image is first quantized into 16 grayscale levels. Then co-occurrence matrices are constructed from four angles $(0$, 45, 90 and $135^{\circ}$ ), and six separating distances (1, 4, 8, 16, 32, and 64 pixels). For a certain distance, mean and range matrices are computed from the co-occurrence matrices with four different angles. Statistics such as energy (angular second moment), contrast, correlation, variance, inverse difference moment, sum entropy, entropy, and difference entropy [59], are calculated from the mean and range matrices. They are used as features. For each separating distance, there are eight features.

Both linear and exponential incremental distances are studied for edge frequency. For uniform incremental distance, one to 40 pixels with incremental distances of one are used, which ends up with 80 features. For exponential incremental distances, the distances of $1,2,4,8,16,32$, and 64 pixels are used. Both four direction and two direction formulations are used, which correspond to 28 and 14 features respectively.

For run length method, input images are first quantized into 16 gray levels. Run length matrices are computed from four different angles $\left(0,45,90\right.$ and $\left.135^{\circ}\right)$. Statistics 
from these matrices are used as features, which include short run emphasis, long run emphasis, gray level nonuniformity, run length nonuniformity, run percentage [52], low gray-level run emphasis, high gray-level run emphasis [22], short run low graylevel emphasis, short run high gray-level emphasis, long run low gray-level emphasis, and long run high gray-level emphasis [29]. Two tests are conducted. The first one only uses Galloway's features, which has feature length of 20 for each image. The other one uses all the features described above, which has feature length of 44 .

Two pattern spectrum methods are tested. The first one is followed by Vincent [162], which uses line-opening/closing spectrum and pseudo-disk openin g/closing spectrum. Each spectrum has 40 elements, which ends up with feature length of 160 in total. The second one is extended from Meijster and Wilkinson [114], which uses horizontal opening/closing spectrum, vertical opening/closing spectrum, area opening/closing spectrum. Each spectrum has 60 elements, which ends up with 240 feature length in total.

The Haar wavelets are used to generate a multi-scale representation of the images. The mean and standard deviation are calculated from the decomposed images. A multi-scale of level from 1 to 7 are tested, which has the feature length of $8,16,24$, $32,40,48$, and 56 respectively.

Six morphological measurements are used as shape-based features, which include: (1) a shape factor based on the perimeter and area of the object; (2) a ratio of maximum and minimum principal moments of the object; (3) a ratio of longest and shortest dimension of the bounding box surrounding the object; (4) a ratio of width at center of object to shortest dimension of the bounding box; (5) a ratio of left $1 / 4$ width of the object to shortest dimension of the bounding box; (6) a ratio of right 1/4-width of the object to shortest dimension of the bounding box [34, 74].

The Ohio State University support vector machine (OSU-SVM) is used to classify the feature vectors. The OSU-SVM was developed by J. Ma, Y. Zhao, and S. Anhalt for Matlab platform using Chang and Lin's LIBSVM algorithm. It is available at http://www/ece.osu.edu/ maj/osu_svm. The OSU-SVM uses decomposition in its optimization and a pair-wise approach to do multi-class classification. In this exper- 
iment, the Gaussian radial basis function (RFB) is used. The Gaussian RBF kernel is defined as

$$
k(x, y)=\exp \left(-\frac{\|x-y\|^{2}}{2 \sigma^{2}}\right)
$$

where $\sigma$ is a scalar value.

A subset of field-collected plankton images, which includes 450 samples for each taxon, is randomly picked from the data set described in Chapter 1 . This data set is used to compare the different pattern representation methods above. Out of this data set, 200 randomly-selected samples per taxon are used for training a support vector machine classifier, and another 200 randomly-selected samples per taxon (without replacement) are used to test the classifier. A $7 \times 7$ confusion matrix is built from these testing samples. The above process is repeated 10 times, so that 10 such confusion matrices are built. The mean and standard confusion matrices are obtained from these 10 independent tries.

\subsection{Results and discussion}

The mean and standard deviation of classification accuracy for each taxon from nine different feature representation methods are shown in Table 4.1, 4.2 and are also plotted in Figure 4-1. The major classification accuracy difference between shape-based feature methods and texture-based feature methods suggests texture-based methods are more suitable to classify plankton images from the field. The overall classification rate of texture-based methods ranges from $65 \%$ to $74 \%$, whereas that of shape-based methods ranges from $39 \%$ to $48 \%$. The pattern spectrum and wavelet transform methods are both shape and texture sensitive. Not surprisingly, their performance lies between these two method groups. Among all the feature representation meth-

ods, the co-occurrence matrices method has the best performance of $74 \%$, while the moment invariants method has the lowest performance of $39 \%$ (Table 4.1).

The reason of the performance difference of these two method groups is due to the nature of data acquisition. Field-collected images impose extra challenges on classification, such as wide view point changes, occluded images, and non-uniform il- 
Table 4.1: Mean classification accuracy from different feature representation methods, where the unit is in percent. The abbreviations are as follows: MI - moment invariants, FD - Fourier descriptors, SS - shape spectrum, MM - morphological measurements, CM - co-occurrence matrices, RL - run length, EF - edge frequency, PS pattern spectrum, WT - wavelet transform. The best performance for single feature method is the co-occurrence matrices method, which has the average of classification accuracy of $74 \%$. It is clear to see that the texture-based methods are superior than shape-based methods.

\begin{tabular}{||lllllllllr||}
\hline \hline Taxonomic group & MI & FD & SS & MM & CM & RL & EF & PS & WT \\
\hline Copepod & 24 & 41 & 47 & 39 & 70 & 66 & 49 & 60 & 62 \\
Rod-shaped diatom chains & 72 & 81 & 79 & 88 & 90 & 84 & 90 & 85 & 87 \\
Chaetoceros chains & 16 & 50 & 52 & 21 & 77 & 67 & 69 & 60 & 70 \\
Chaetoceros socialis chains & 77 & 59 & 67 & 60 & 85 & 75 & 76 & 72 & 73 \\
Hydroid medusae & 33 & 29 & 63 & 61 & 76 & 70 & 75 & 62 & 70 \\
Marine snow & 37 & 27 & 50 & 19 & 68 & 65 & 53 & 50 & 48 \\
Other & 10 & 36 & 27 & 51 & 45 & 41 & 47 & 41 & 38 \\
\hline Average accuracy & 39 & 46 & 48 & 48 & 74 & 67 & 65 & 61 & 64 \\
\hline \hline
\end{tabular}

Table 4.2: Standard deviation of classification rates from different feature representation methods, where the unit is in percent. The abbreviations are same as Table 4.1.

\begin{tabular}{||lllllllllr||}
\hline \hline Taxonomic group & MI & FD & SS & MM & CM & RL & EF & PS & WT \\
\hline Copepod & 5.3 & 3.9 & 3.3 & 4.8 & 3.0 & 5.5 & 3.2 & 5.0 & 4.0 \\
Rod-shaped diatom chains & 3.4 & 2.8 & 2.5 & 2.2 & 1.3 & 4.4 & 1.4 & 1.9 & 2.7 \\
Chaetoceros chains & 9.9 & 3.4 & 3.6 & 2.5 & 1.8 & 3.7 & 3.1 & 4.6 & 2.9 \\
Chaetoceros socialis chains & 3.0 & 3.6 & 3.1 & 3.8 & 2.5 & 3.3 & 4.3 & 2.3 & 2.3 \\
Hydroid medusae & 3.4 & 3.1 & 1.5 & 3.1 & 2.8 & 3.1 & 4.0 & 4.5 & 4.9 \\
Marine snow & 12.1 & 3.1 & 2.3 & 4.3 & 3.0 & 3.9 & 3.1 & 2.4 & 4.1 \\
Other & 7.0 & 2.9 & 3.6 & 3.4 & 2.8 & 3.6 & 3.7 & 4.3 & 1.8 \\
\hline \hline
\end{tabular}



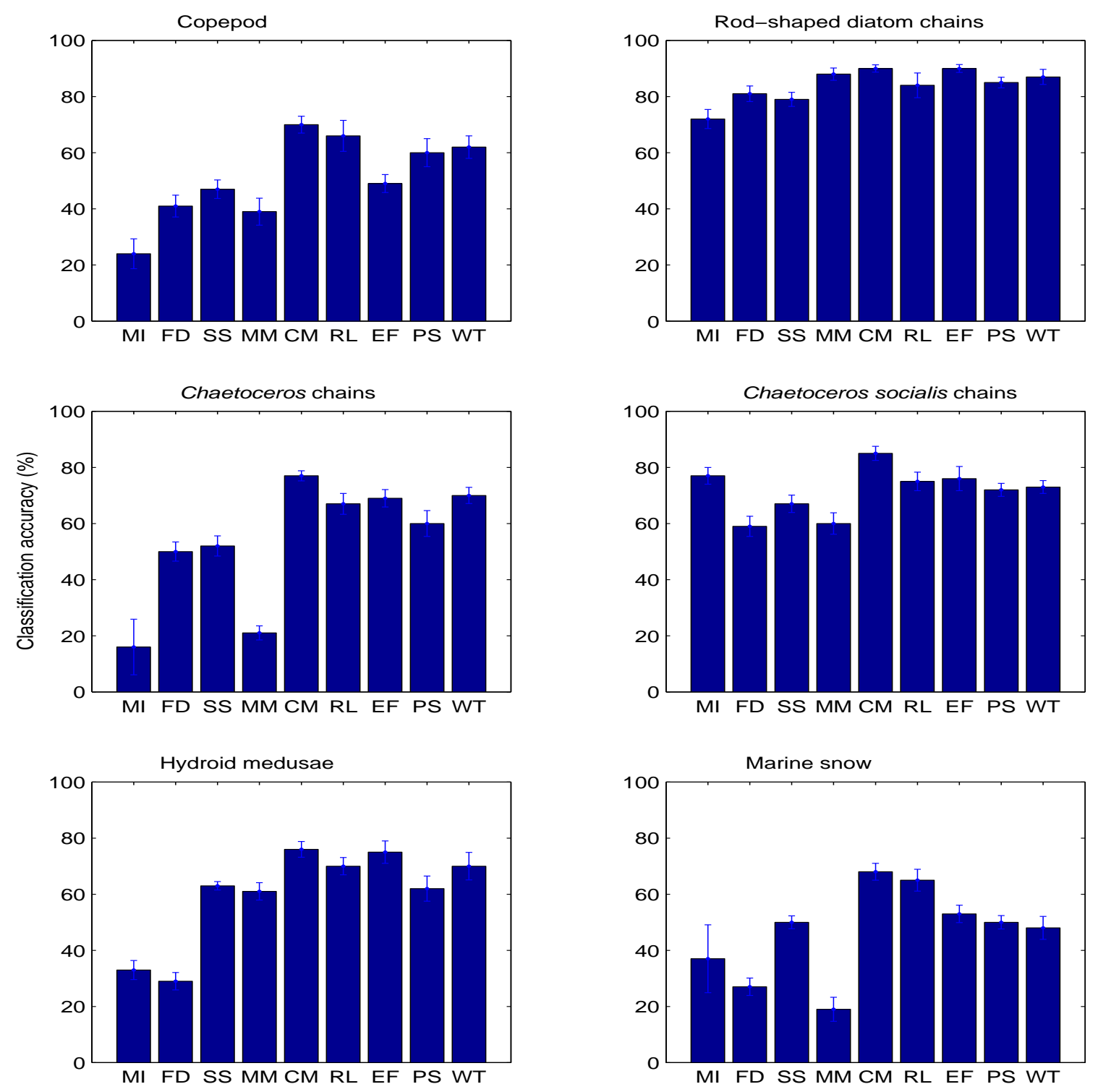

Figure 4-1: Mean and standard deviation of classification accuracy from different feature presentation methods for each taxon. The abbreviations are as follows: MI moment invariants, FD - Fourier descriptors, SS - shape spectrum, MM - morphological measurements, CM - co-occurrence matrices, RL - run length, EF - edge frequency, PS - pattern spectrum, WT - wavelet transform. It clearly shows the jump between shape-based features and texture-based features. The pattern spectrum and wavelet transform methods are between shape-based and texture-based methods, their performances lie in between these two group of methods. 
lumination, as well as traditional challenges (RST invariance) on classification. Nonuniform illumination makes perfect segmentation much hard or even impossible. It is illustrated in Figure 4-2. Furthermore, the dataset used for training and testing classifiers in this chapter is a random-picked subset of real world data instead of a hand-picked subset. The difference between random-picked and hand-picked samples is that human operators tend to pick "easy" (distinctive) samples, which makes the classification performance estimates based on hand-picked samples optimistically biased. Notice the "other" category in the Tables 4.1, and 4.2, which may not exist in most hand-picked training samples. It was discussed in Davis et al.[34] that including "other" as a category in the classifier may decrease the classification accuracy more than $10 \%$.

The sensitivity of the training samples is shown in Table 4.2. Moment invariants are very sensitive to switching the training and testing samples, while co-occurrence matrices are more robust to such changing. In other words, the co-occurrence matrices method ranks top in both classification accuracy and sensitivity.

\subsubsection{High order moment invariants}

The short feature length may be the cause of relative low performance of the moment invariants method. Hu's moment invariants [72] are used in Figure 4-1. High order of moment invariants discussed by Li [101] are investigated. Moment invariants of order up to 7 are calculated and compared with Hu's moment invariants. The results are summarized in Figure 4-3. High order moment invariants behave as poorly as low order moment invariants. There is only a slight classification accuracy improvement

by using high order moment invariants, which is not statistically significant. There is no benefit to use high order moment invariants in this dataset. 


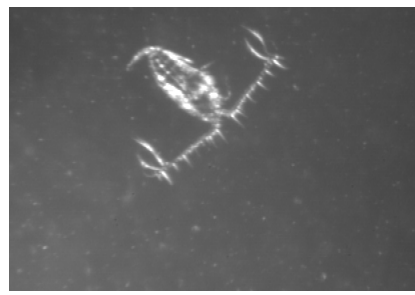

(a) original image

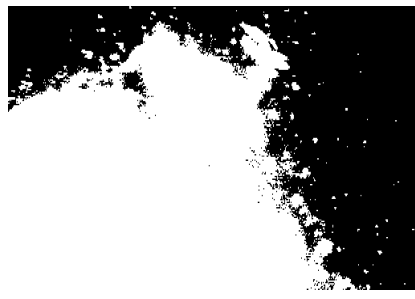

(c) segmented image of (a)

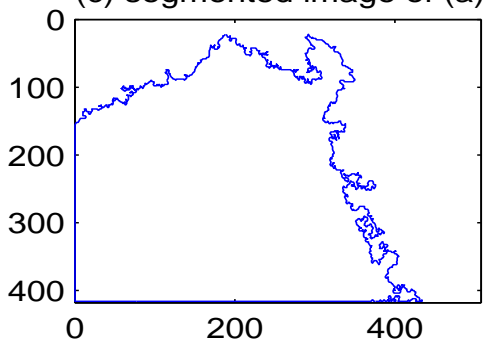

(e) contour of the largest object from (c)

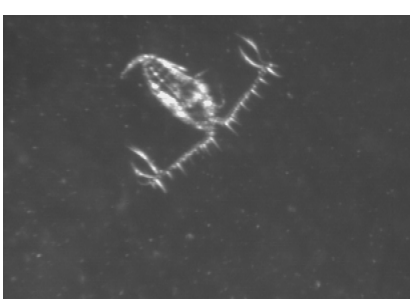

(b) image after gradient removal

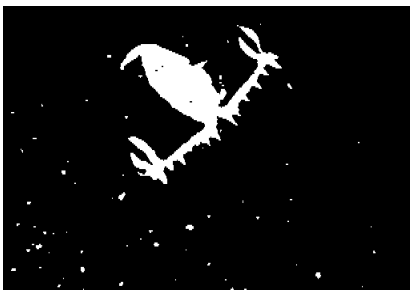

(d) segmented image of (b)

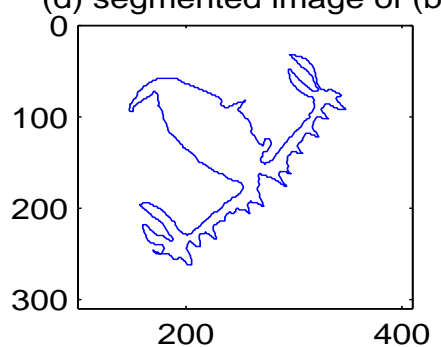

(f) contour of the largest object from (d)

Figure 4-2: Illustrates the problem of non-uniform illunimation on segmentation. (a) the original image, (b) gradient correction of (a), (c) segmentation of (a), (d) segmentation of (b), (e) contour of the largest object from (c), (f) contour of the largest object from $(\mathrm{d})$ 

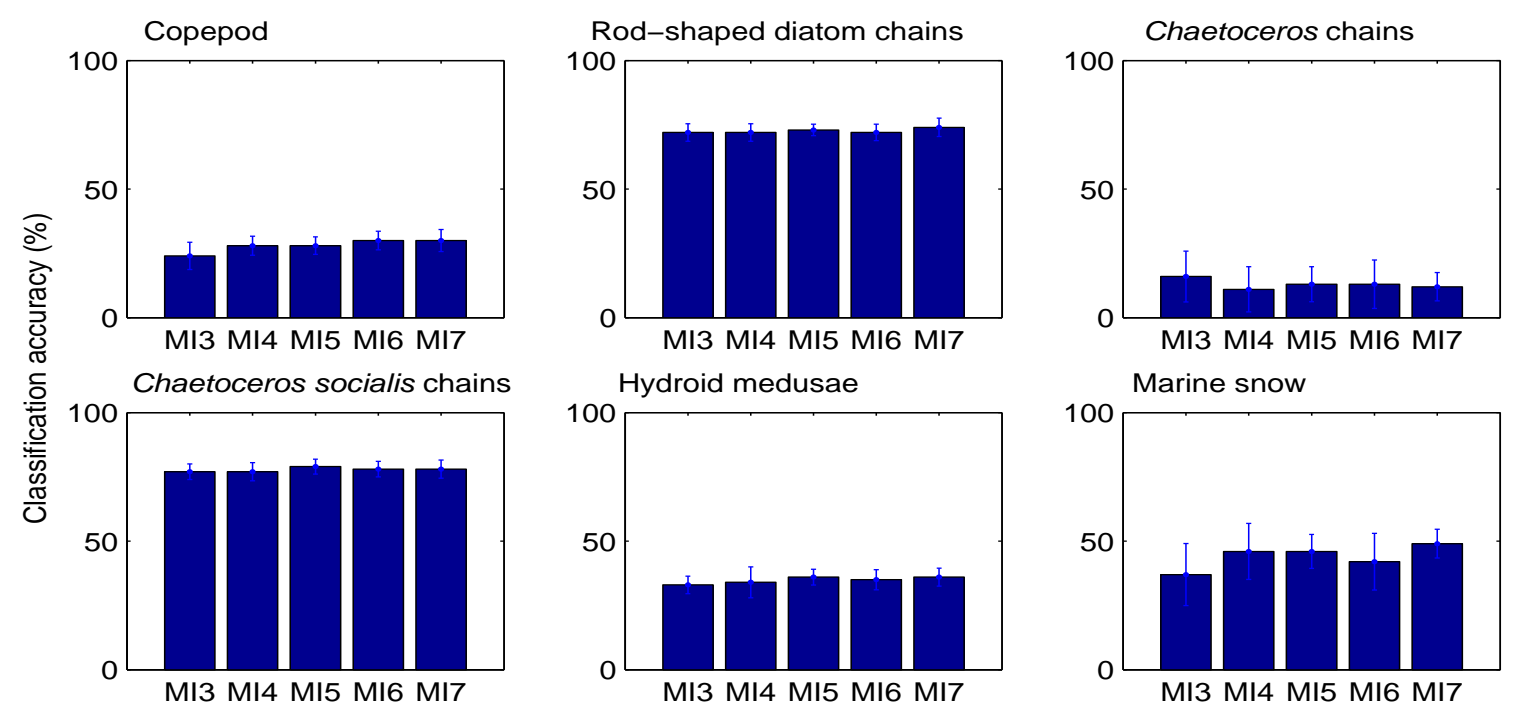

Figure 4-3: Mean and standard deviation of classification accuracy for moment invariants of different orders for each taxon. MI3-7 stands for moment invariants up to order 3-7, which correspond to feature length of 7, 12, 18, 24, and 33 respectively. where MI3 is equivalent to Hu's moment invariants. There is no benefit to using high order moment invariants in this dataset.

\subsubsection{Radial Fourier descriptors vs. complex Fourier descrip- tors}

Radial Fourier descriptors are used in Figure 4-1 to characterize a contour function. A radius-vector function is defined as the distances from reference point (usually the centroid of the object) to the contour in the direction of $\theta$-ray, where $0 \leq \theta \leq 2 \pi$. Radius-vector functions are only suited for representing star-shaped contours. Most plankton images do not have star-shaped contours. The problem of using a radiusvector function to encode a non-star-shaped contour is shown in Figure 4-4. The recovered contour from radius-vector function is nothing close to the original contour of the largest object.

In this section, we investigate the effect of using radial Fourier descriptors for plankton images by comparing with complex Fourier descriptors (Figure 4-5). Consider a closed contour of an object in a complex plane, every point on the contour is parameterized by its complex coordinates. When moving a point along the contour in 


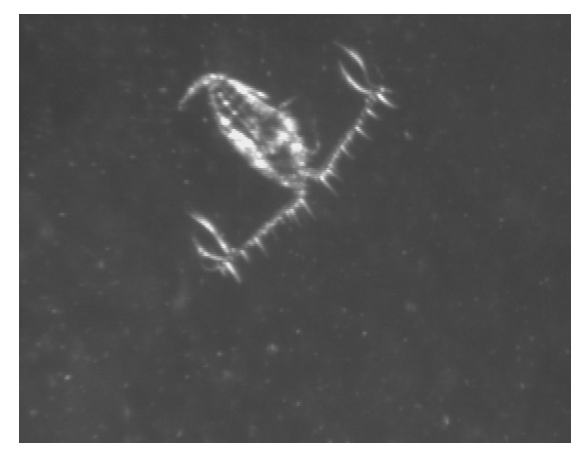

(a) original image

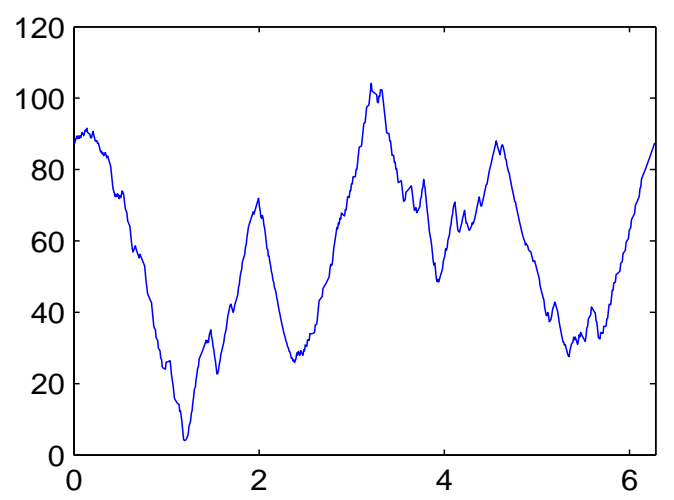

(c) radius-vector function

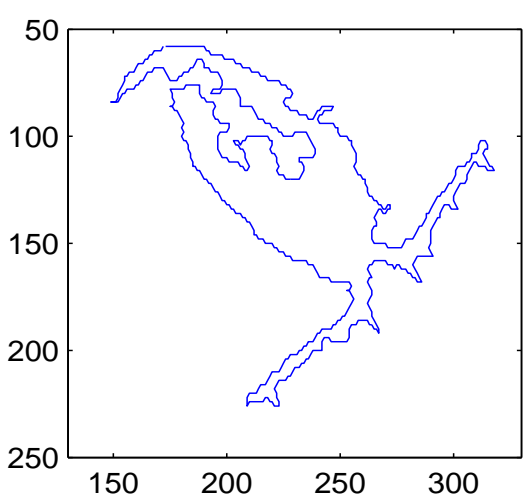

(b) contour of the largest object

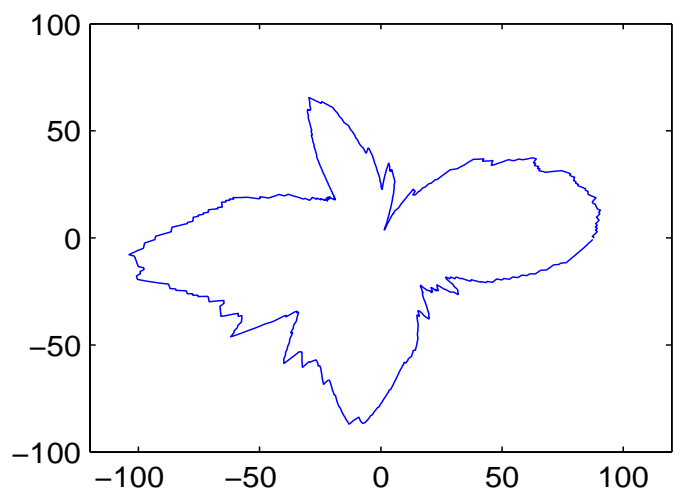

(d) recovered contour from radius-vector function

Figure 4-4: Illustrates the problem of using a radius-vector function to encode a nonstar-shaped plankton image. (a) the original image, (b) the contour of the object, (c) the radius-vector function from the contour model of (b), (d) the recovered contour of the object based on radius-vector function (c) with assumption that the object is star-shaped. 

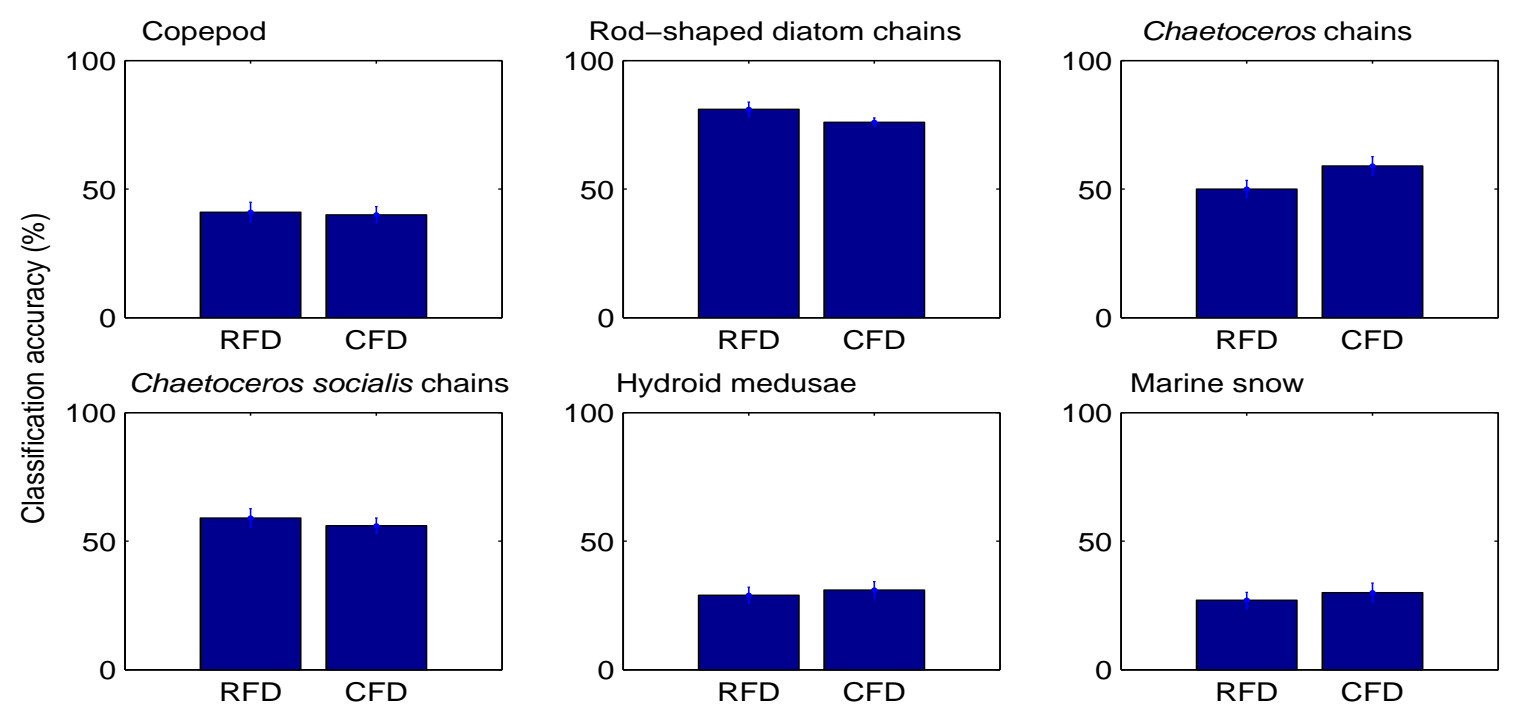

Figure 4-5: A comparison between radial Fourier descriptors (RFD) and complex Fourier descriptors (CFD).

the counterclockwise direction, we generate a complex function of the contour. The normalized coefficients of Fourier transform of the complex function are the complex Fourier descriptors.

There is almost no difference in classification accuracy between radial Fourier descriptors and complex Fourier descriptors, which suggests different contour parameterization is less important than the quality of contour models.

\subsubsection{Co-occurrence matrices}

A number of experiments is conducted on the co-occurrence matrices method. First, the different multi-scale level effect is investigated. The results are summarized in Figure 4-6. Co-occurrence matrices features from one to six multi-scale levels are tested, which corresponds to 1, 4, 8, 16, 32, and 64 pixel separation distances. Exponential incremental distances are used since I find there is too much redundant information in neighboring distance for linear incremental case (refer to Figure 49). The classification accuracy rises sharply with more separating distances added at first, and reaches top performance when 3-4 multi-scale levels are used. Then the 
classification performance drops slightly when more multi-scale levels are included in the feature set. The reason for the classification accuracy drop is that the images used are of relatively small size. With longer separating distance, such as 64 pixels, a considerable number of images has one or both dimensions shorter than this distance, resulting in no useful information being measured. For the rest of the chapter, four separating distances are used if it is not explicitly mentioned.
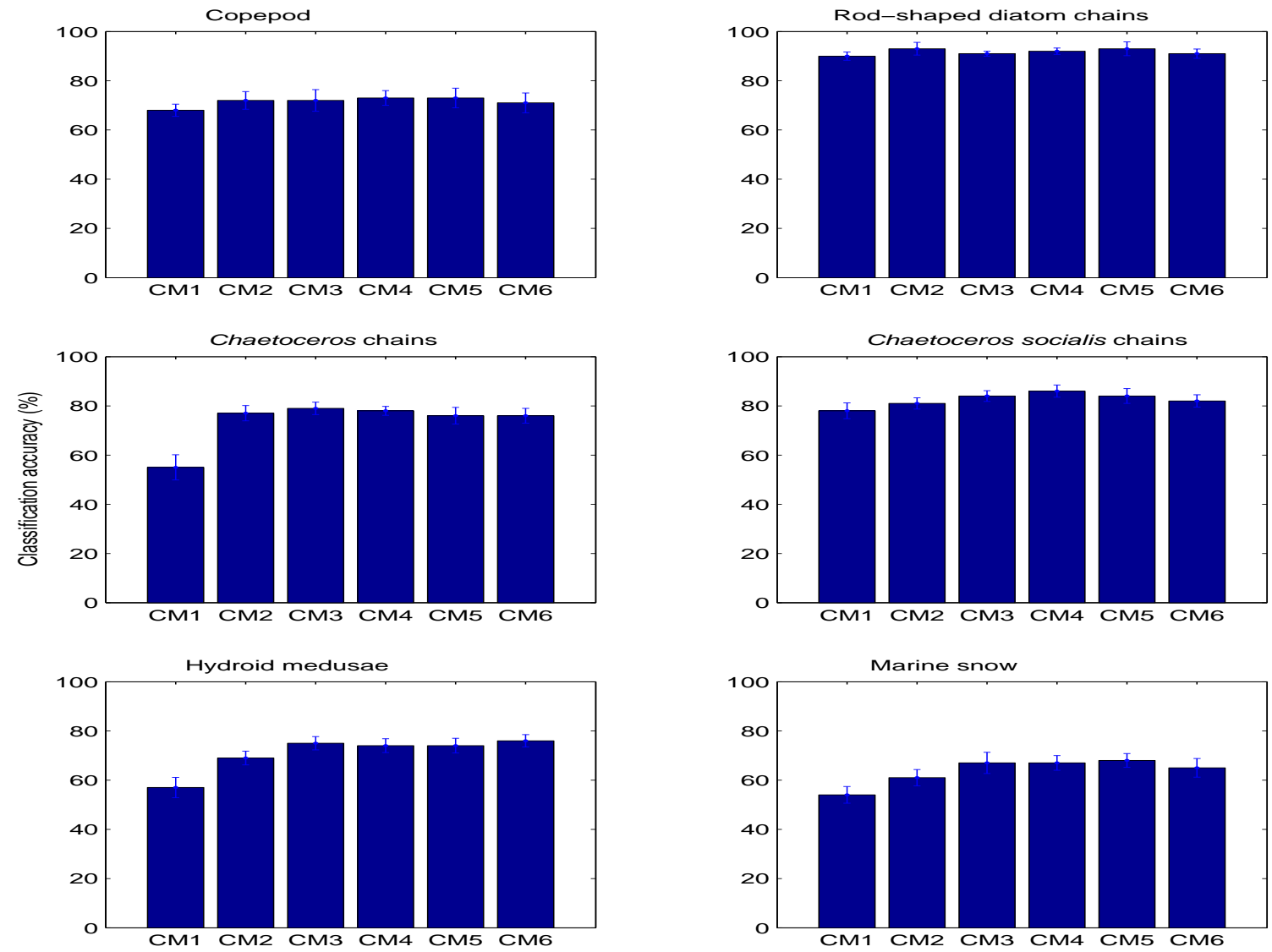

Figure 4-6: Mean and standard deviation of classification accuracy for co-occurrence matrices of different multi-scale levels for each taxon. The abbreviations CM1-6 stand for co-occurrence matrices of multi-scale levels from 1 to 6 , which correspond to feature length of $16,32,48,64,80$, and 96 respectively. The classification accuracy first rises sharply with an increase of multi-scale levels, and reaches top performance with 3-4 multi-scale levels. The performance then drops down slightly as more multiscale levels are included in the feature set.

Next, a comparison is done by using co-occurrence matrices themselves as features versus using statistical measurements from them. For each image which is quantizated 
into 16 gray levels, the size of each co-occurrence matrix is $16 \times 16$. When four multi-scale levels are used, there are a total of eight mean and range co-occurrence matrices, which results in total feature length of 2048. From Figure 4-7, there is not much accuracy difference between raw co-occurrence matrices and statistics from these co-occurrence matrices. However, from the classification point of view, short feature length is preferred. The high classification accuracy of raw co-occurrence matrices suggests that the support vector machine classifier can "smartly" find out relevant information in the co-occurrence matrices. On the other hand, since the classifier is not designed to do feature extraction, there still is room for improvement in classification accuracy with the co-occurrence matrices method.
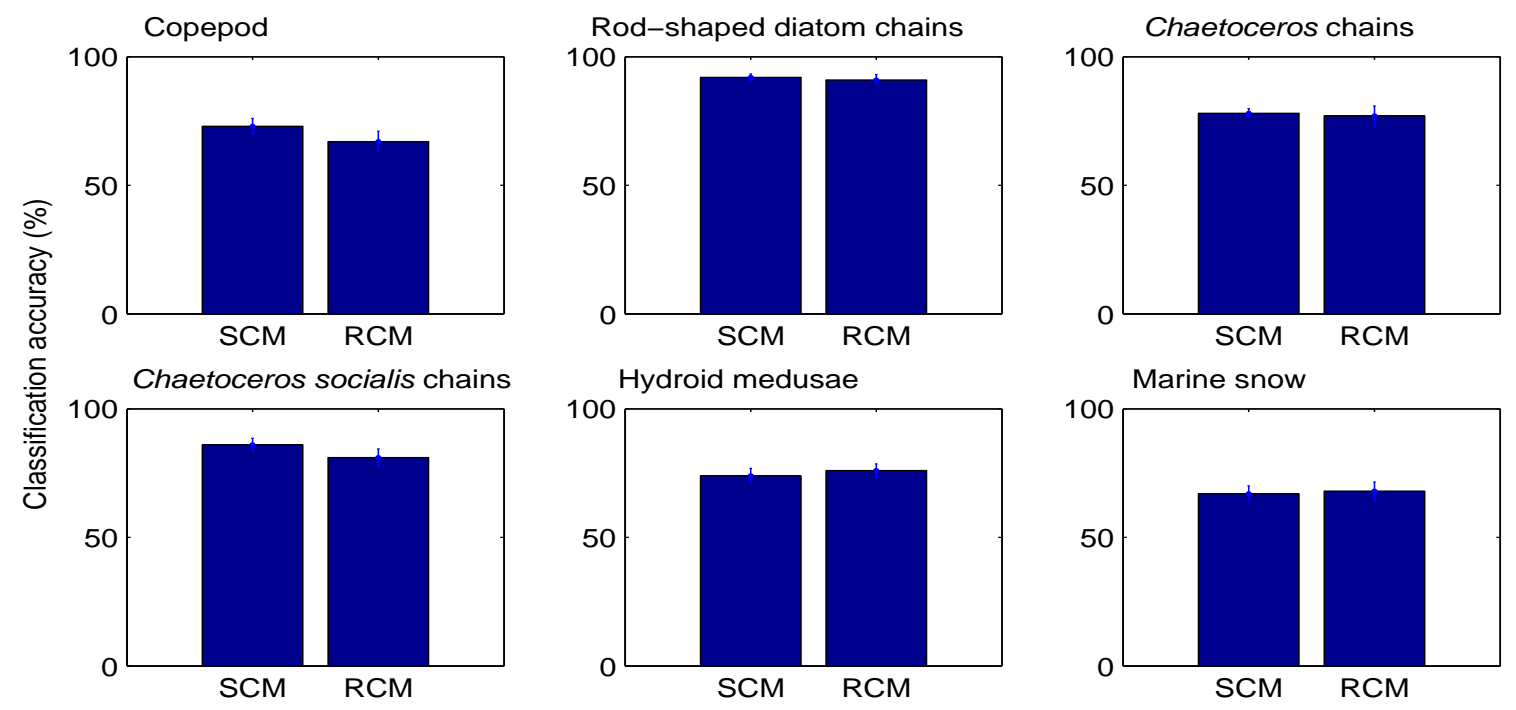

Figure 4-7: A comparison between raw co-occurrence matrices (RCM) and statistics of co-occurrence matrices (SCM). There is little difference between SCM and RCM.

The virtual support vector machine is investigated by utilizing the co-occurrence matrices features. The idea of the virtual support vector machine is that we can achieve transformation invariance by expanding original training samples by adding artificial training samples. The artificial training samples are generated by transforming the original training samples accordingly to the invariance of interest, such as rotation or scaling. By training the classifier with both original and artificial 

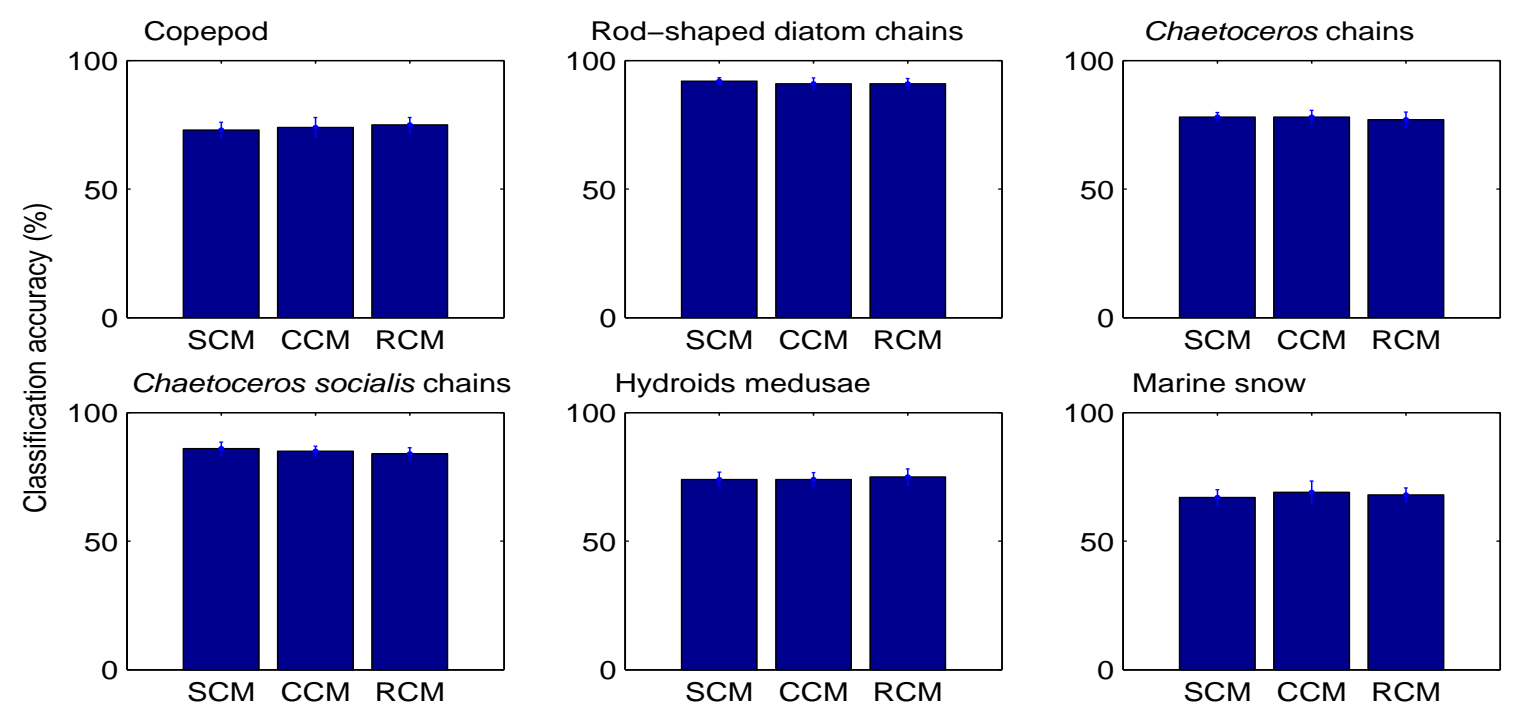

Figure 4-8: Virtual support vector machine test on the co-occurrence matrices method. SCM - statistics of co-occurrence matrices, CCM - statistics of co-occurrence matrices from original image and its complement, RCM - statistics of co-occurrence matrices from original image and resized images of 0.8 and 1.2. No accuracy gain is obtained by adding virtual samples in the training set.

samples with enough time, the hope is that the classifier will achieve transformation invariance from the samples. Figure 4-8 is the result of the virtual support vector machine test. There is no improvement in expanding the original training samples to its complement and its resized version, which suggest that the co-occurrence matrices feature has already achieved such invariance.

\subsubsection{Edge frequency}

The exponential distance interval spectrum works better than the linear distance interval spectrum, while four direction formulation works better than two direction formulation (Figure 4-9). The average classification accuracies of the linear distance interval, the exponential distance interval with two directions, the exponential distance interval with four directions are $56.7 \%, 60.9 \%$, and $65.5 \%$ respectively. The classification accuracy for interested taxon ranges from $34.4 \%$ to $84.3 \%$ for linear distance interval, from $41.7 \%$ to $88.3 \%$ for two direction formulation, and from $49.0 \%$ 
to $89.5 \%$ for four direction formulation.
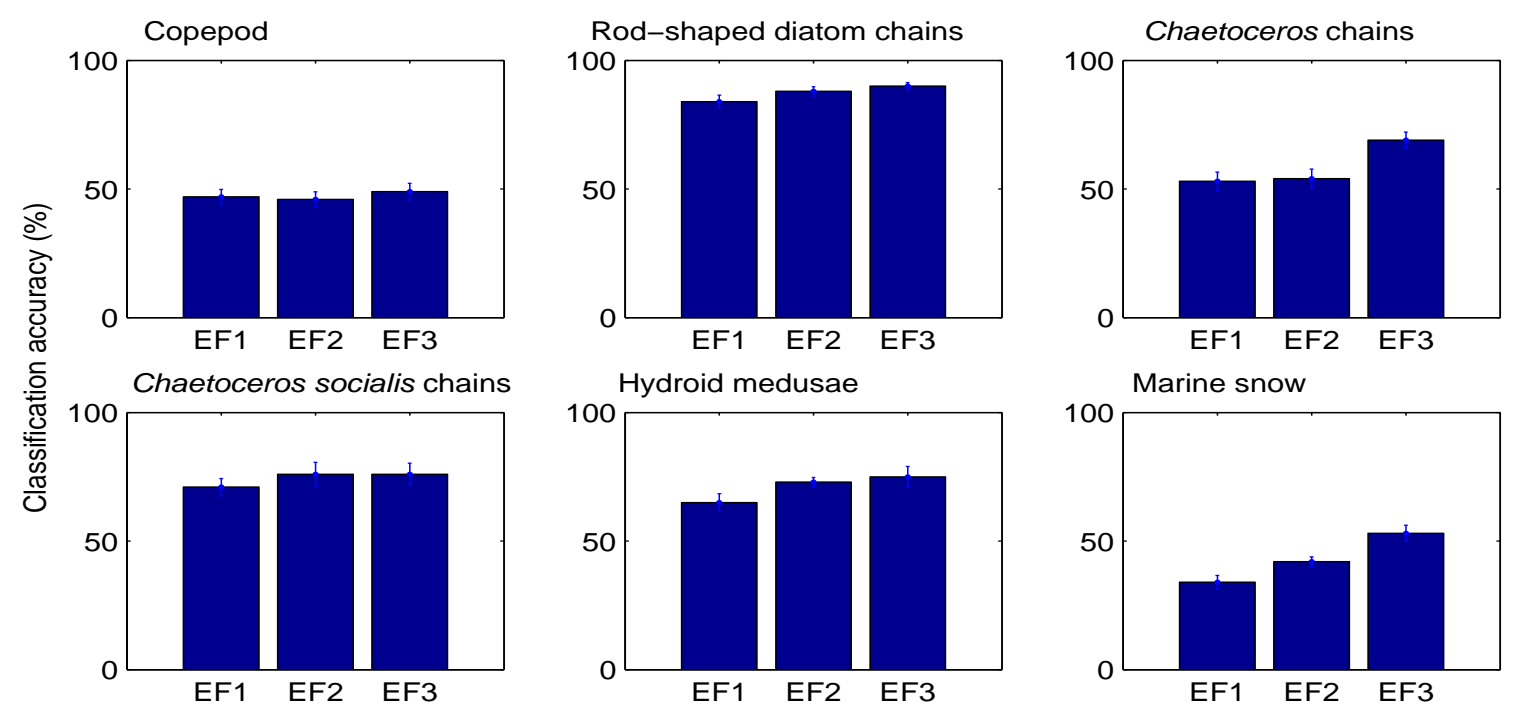

Figure 4-9: A comparison of edge frequency features, where EF1 is EF spectrum with linear distance interval from 1 to 40 pixels and two directions formula (horizontal \& vertical, and diagonals), EF2 is EF with 7 exponential distance interval from 1 to 64 and two directions formula (horizontal \& vertical, and diagonals), EF3 is EF with 7 exponential distance interval from 1 to 64 and four directions formula (horizontal, vertical and two diagonals). It is clear that exponential distance interval works better than the linear distance interval, and four direction formula works better than two direction formula.

\subsubsection{Run length}

Basic run length statistics proposed by Galloway [52], and run length statistics extended by Chu et al. [22], and Dasarathy \& Holder [29] are investigated (Figure 4-10). The extended statistics have an average accuracy of $66.7 \%$, while basic statistics have an average accuracy of $60.6 \%$. The classification accuracy for interested taxon ranges from $57.0 \%$ to $78.4 \%$ for basic statistics, while from $64.5 \%$ to $83.6 \%$ for extended statistics. 

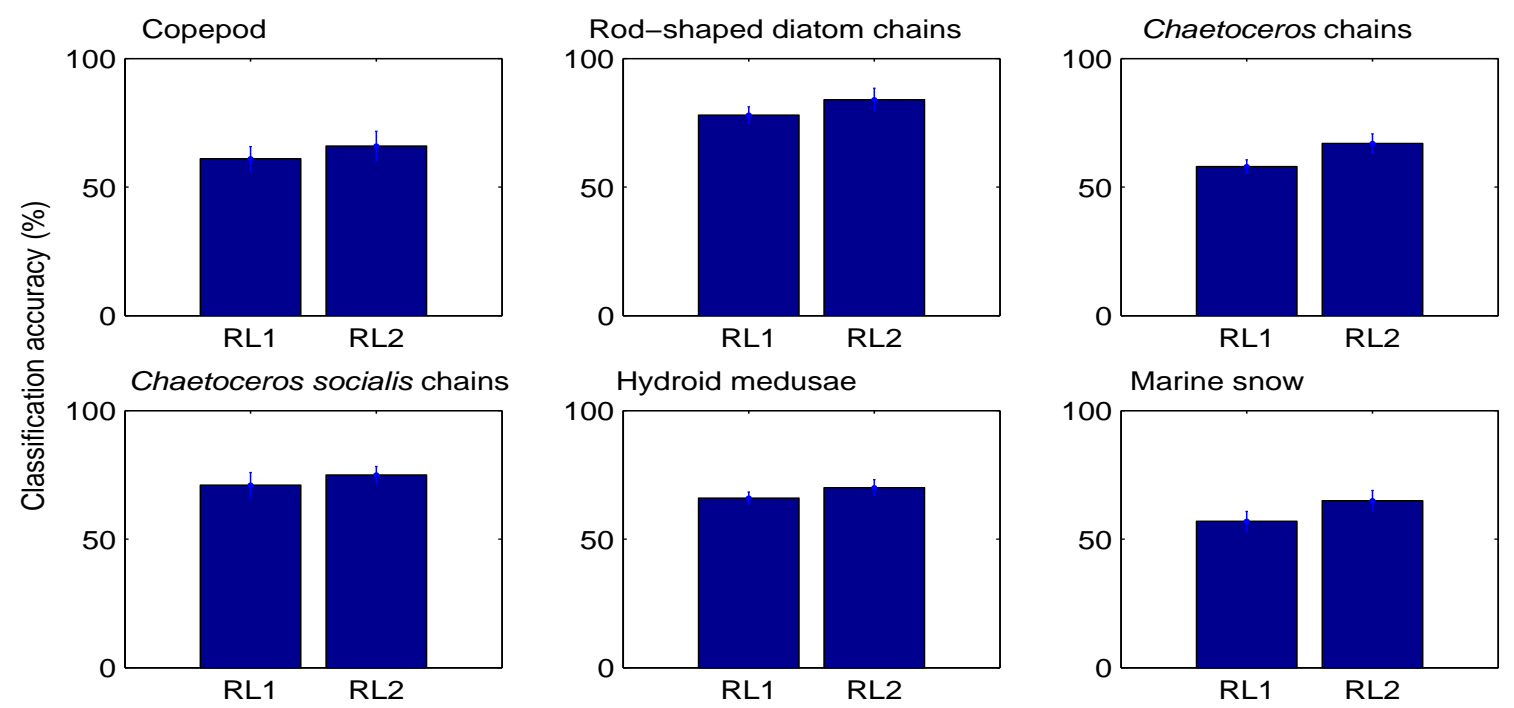

Figure 4-10: Comparison of run length methods. RL1 - run length statistics proposed by Galloway, 5 statistics from each run length matrix, total 20 features for 4 directions. RL2 - extended run length statisitcs by Chu et al., and by Dasarathy and Holder. 11 statistics from each run length matrix, total 44 features for 4 directions. The extended features give a slight better performance for all the taxa.

\subsubsection{Pattern spectrum}

Pattern spectrum as implemented by Vincent [162] (PS1) is compared with that implemented by Meijster and Wilkinson [114] (PS2). PS2 is extended to include line opening/closing spectra as well as area opening/closing spectra. PS1 outperforms PS2 on all the taxa except marine snow. The average classification accuracies for PS1 and PS2 are $61.4 \%$ and $52.2 \%$ respectively. The classification accuracy for interested taxon ranges from $49.8 \%$ to $85.3 \%$ for PS1, while from $36.9 \%$ to $77.8 \%$ for PS2 (Figure $4-11$ ).

\subsubsection{Wavelet transform}

The classification accuracies for wavelet transform feature increase with use of more multi-scale level at first, then change a little after 3-4 multi-scale levels are considered (Figure 4-12). The average classification accuracy changes from $59.2 \%$ to $64.3 \%$ from WL1 to WL7. 

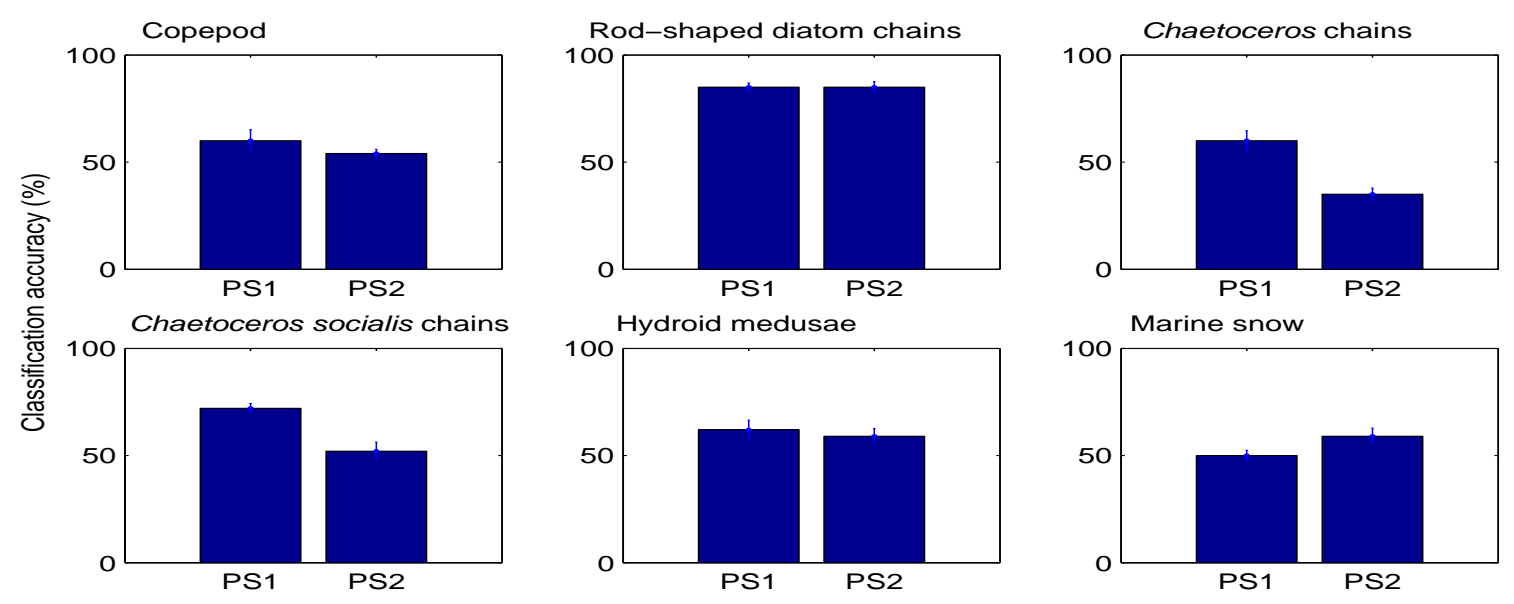

Figure 4-11: Comparison between two implementations of pattern spectrum. PS1 PS by Vincent, linear and pseudo opening and closing spectra, each has 40 elements, total feature length of 160. PS2 - PS modified from Meijster and Wilkinson, horizontal and vertical line opening and closing spectra, and area opening and closing spectra, each has 40 elements, total feature length of 240. PS1 outperforms PS2 on all the taxa except marine snow.
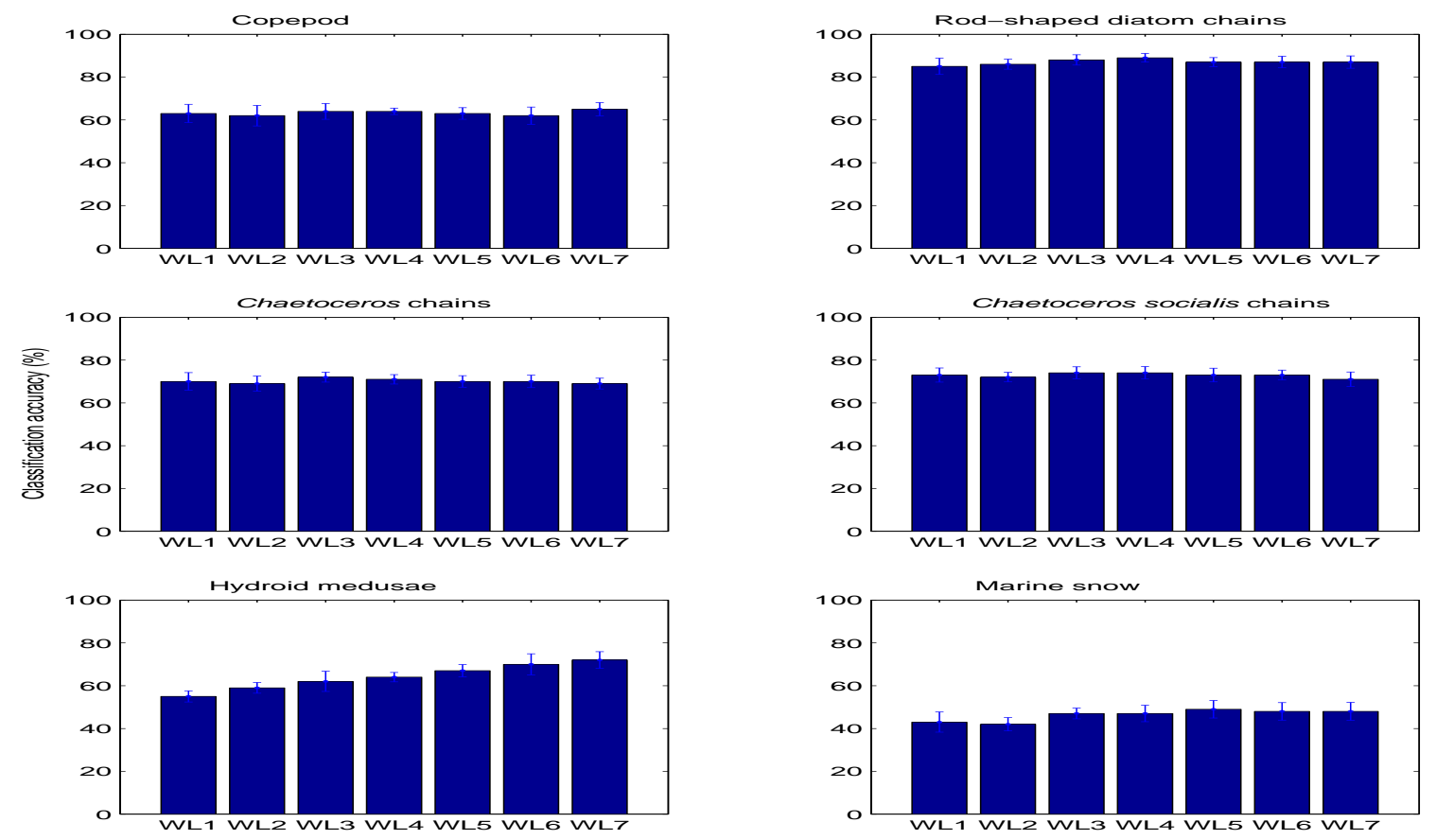

Figure 4-12: Multi-scale level test for wavelet transform features. WL1-7 stands for features from wavelet transform with multi-scale level from 1 to 7 . 


\subsection{Conclusion}

Texture-based features are more important to classify field-collected plankton images than shape-based features, even though shape-based features are extensively used in the literature.

Multi-scale representation of texture features helps to improve the distinctive power of texture features. Exponential incremental distance works better than linear incremental distance. The optimal multi-scale level depends on the resolution and size of the images. For this dataset, the optimal multi-scale levels are 3-4 levels.

Multi-scale co-occurrence matrices work best among all the feature methods tested. The mean classification accuracy of $73 \%$ for seven taxa on independent testing data sets is achieved using four multi-scale co-occurrence matrices. 


\section{Chapter 5}

\section{Co-Occurrence Matrices and Support Vector Machine}

In the previous chapter, I compared different feature presentation methods, and demonstrated that multi-scale texture-based pattern presentation methods are more suitable to classify field-collected images. In this chapter, I apply these findings and develop a classification method, which utilizes texture-based features, multi-scale cooccurrence matrices, and a support vector machine (COM-SVM) to classify the whole data set and estimate the abundance of 6 major taxonomic groups. Such results are compared against previous classification system with combined shape-based features and neural network(CSF-NN) classifier[34]. Using texture-based features calculated from multi-scale co-occurrence matrices alone reduces the classification error rate from 39 to $28 \%$. Subsequent plankton abundance estimates are improved by more than $50 \%$ in regions of low relative abundance. This chapter was published in Marine Ecology Progress Series[73].

This chapter is organized as follows. In Section 5.1, I describe the co-occurrence matrices method. In Section 5.2, I discuss the support vector machine method. In Section 5.3, I describe the feature extraction and classification used in this chapter. The classification results are given in Section 5.4, followed by the conclusion in Section 5.5. 


\subsection{Co-Occurrence Matrices}

Spatial gray level co-occurrence provides second-order statistics from the images. Julesz [85] first used first-order and second-order statistics in texture discrimination. The co-occurrence method was first proposed by Haralick et al. [59] as a texture feature and it has been widely used thereafter. It is based on estimation of the joint probability distribution of pairs of pixels with gray level $i$ and $j$, spatial distance $d$ and angle $\theta$ in an image. Each element in the co-occurrence matrix is the occurrence of pairs of pixels having gray levels $i$ and $j$ and a certain spatial relationship in the whole image (i.e., distance $d$ and angle $\theta$ ). Thus, for an image of $L$ quantization level, the size of its co-occurrence matrix is $L \times L$. The number of co-occurrence matrices is dependent on the number of different separation distances and quantized levels of the angle. For computation efficiency, the angle is usually quantized to $45^{\circ}$ or $90^{\circ}$. It is hard to select $d$ without prior information. It is common to choose $d=1$. In my experiment, I have quantized angle to $45^{\circ}$, which resulted in 4 different angles $\left(0,45,90\right.$, and $\left.135^{\circ}\right)$, and chose $d=1,4,8,16$ pixels.

\subsection{Support Vector Machines}

Support Vector Machines (SVM) were proposed by Vapnik [160, 161] and have yielded excellent results in a variety of data classification tasks. It is primarily a two-class classifier and involves two steps. First, the feature vectors, $\mathbf{x}$, of the training samples are mapped into a high (potentially infinite) dimensional space, $\mathcal{H}$. A hyperplane then is constructed in order to separate the training samples in $\mathcal{H}$. Different mappings $\mathbf{x} \mapsto \mathbf{\Phi}(\mathbf{x}) \in \mathcal{H}$ construct different SVMs.

The mapping $\Phi(\cdot)$ is performed by a kernel function $K(\cdot, \cdot)$ which defines an inner (dot) product in $\mathcal{H}$. The decision function (i.e., the hyperplane), $f$, given by an SVM is

$$
f(\mathbf{x})=\operatorname{sgn}(<\mathbf{w}, \Phi(x)>+b)=\operatorname{sgn}\left(\sum_{i=1}^{m} \alpha_{i} y_{i} \mathbf{K}\left(x_{i}, x\right)+b\right),
$$

where $\mathbf{w}$ and $b$ define the orientation and translation of $f$, respectively, $i$ is the 
training sample index, $y$ is class label, and $\alpha$ is a scalar.

The goal in training an SVM is to find the separating hyperplane which has the maximal distance to the closest training samples in space $\mathcal{H}$. This distance is called the margin. These particular training feature vectors used to determine optimal hyperplanes are called support vectors. In order to cope with non-separable cases, a set of slack variables $\xi_{i} \geq 0$ are introduced. If there are $m$ training samples: $\mathbf{x}_{\mathbf{1}}, \mathbf{x}_{\mathbf{2}}, \cdots, \mathbf{x}_{\mathbf{m}}$ with class label $y_{i} \in\{-1,1\}$, the classification reduces down to the following optimization problem:

$$
\text { minimize } L_{P}(\mathbf{w}, \xi)=\frac{1}{2}\|\mathbf{w}\|^{2}+\frac{C}{m} \sum_{i=1}^{m} \xi_{i},
$$

with relaxed separation constraints,

$$
y_{i}\left(<\phi\left(\mathbf{x}_{\mathbf{i}}\right), \mathbf{w}>+b\right) \geq 1-\xi_{i}, \quad i=1, \cdots, m
$$

where $\mathbf{w}$ is normal to the hyperplane, $C$ is a scalar value that controls the tradeoff between the empirical risk and margin width. The dual formulation is usually easy to solve, and is defined as:

$$
\text { maximize } L_{D}(\alpha)=\sum_{i=1}^{m} \alpha_{i}-\frac{1}{2} \sum_{i, j}^{m} \alpha_{i} \alpha_{j} y_{i} y_{j} \mathbf{K}\left(x_{i}, x_{j}\right) \text {, }
$$

subject to the constraints

$$
\sum_{i=1}^{m} \alpha_{i} y_{i}=0, \quad 0 \leq \alpha_{i} \leq C, \quad i=1, \cdots, m
$$

There are three main ways to extend SVMs from 2-class to multi-class classification: 1) The simplest is the one-versus-all approach [133] in which a set of binary SVMs are trained to separate one class from the rest. The main drawback of this approach is that the sample size is unbalanced, with the number of images in the selected class typically being much less than the number of images not in that class. 2) Another method is the Error-Correcting Output Codes [39], in which a series of 
binary problems are generated from a multiclass problem by splitting the original set of classes into two subsets. This method appears promising but is untested for plankton image data. 3) In the present study, I used a pairwise approach, where all possible pairs of 2 classes were used to build binary SVMs. For the classification with

$N$ classes, $\frac{N(N-1)}{2}$ binary SVMs are needed. This yields 21 binary SVMs for our case of 7 classes.

An important property of SVM is that the complexity of the classifier is characterized by the number of support vectors instead of the dimension of the hyperspace $\mathcal{H}$. As a result, the SVM is less prone to over-fitting than other methods.

\subsection{Feature Extraction and Classification}

Each image was first quantized to 16 levels. The co-occurrence matrices were calculated from 4 different angles $(0,45,90,135)$ and 4 different distances $(1,4,8$, 16 pixels). A frequency normalization was performed by dividing each entry in the co-occurrence matrices by the total number of neighbor pairs. For example, for an image of size $M \times N$, when the relationship between nearest horizontal neighbors is $\left(d=1, \theta=0^{\circ}\right)$, there will be a total of $2 N(M-1)$ nearest horizontal neighbor pairs. For every four matrices with the same distance, the mean and range matrices were calculated. Thus, for each image, eight co-occurrence matrices were computed. The energy, contrast, correlation, variance, inverse-difference moment, and entropy of these matrices[59] were calculated and used as feature vector elements. These features were further normalized to have zero mean and unit standard deviation.

The Ohio State University (OSU) support vector machine (OSU-SVM) was used to classify these feature vectors. The OSU-SVM was developed by Ma, Zhao, and Anhalt for Matlab platform using Chang and Lin's LIBSVM algorithm (Chang \& Lin, 2001). It is available at http://www/ece.osu.edu/ maj/osu_svm. The OSU-SVM uses decomposition in its optimization and a pair-wise approach to do multi-class classification. Different kernels were tested on my data set. In my experiment, the Gaussian radial basis function (RFB) performed best in terms of validation error. 
The Guassian RBF kernel is defined as

$$
k(x, y)=\exp \left(-\frac{\|x-y\|^{2}}{2 \sigma^{2}}\right)
$$

where $\sigma$ is a scalar value.

Two data sets were randomly picked from the working data set. These data sets had 200 samples per taxon and were used to train and validate the SVM classifier, respectively. Values of $\sigma$ and the regularization constant $C$ were optimized based on the classification error found from tests with the validation data set. Values of $\sigma=0.1, C=50$ gave the best classifier performance. Since the validation data set was used to tune the classifier parameters, it is not valid to use them to testify the classifier (i.e., generate confusion matrix). In this study, the classifiers are verified by classifying the whole data set.

\subsection{Classification results}

I compared the performance of my COM-SVM system to the prior plankton classification system described in Chapter $3[150,34]$. The COM-SVM yielded a $28 \%$ reduction in recognition error rate (cf. Table 5.1 and Table 5.2, respectively). The overall performance of the COM-SVM was $72 \%$ compared to $61 \%$ of the previous system. The COM-SVM classifier performed better than the combined shape-based features (moment invariants, Fourier descriptor, granulometry curve, and morphological measurements) and neural network (CSF-NN) classifier for almost all the categories except the "other" category (Tables 5.1 and 5.2). This finding supports my idea that for field-collected samples, texture-based features are more important than global shape-based features in plankton classification, due to occlusion and nonlinear illumination, or projection variance inherent in field-collected images. Most occlusions occur when part of an organism is out of the image volume. Some occlusions happen when part of an organism is darker than the rest because of the the nolinear illumination. The global segmentation only segments part of the organism (cf. 
Figure 4-3). The situation of nonlinear illunimation should be improved by using a ring-illuminator in future instruments. A small amount of occlusion can also occur when an out-of-focus organism is in the light path of an in-focus organism. This situation only occurs when the concentration of the plankton is very high $(\geq 10$ ind. $\left.m l^{-1}\right)$.

Table 5.1: Confusion matrix for EN302, VPR Tow 7, based on the co-occurence matrix classifier using hold-out method. Column and row heading are coded as: C1, copepod; C2, rod-shaped diatom chains; C3, Chaetoceros chains; C4, Chaetoceros socialis colonies; C5, hydroid medusae; C6, marine snow; C7, 'other'; and $P_{d}$, probability of detection. True counts (i.e. human counts) for a given taxa are given in the columns, while counts by automatic identification (i.e. computer counts) are given in the rows. Correct identifications by the computer are given along the main diagonal, while the off-diagonal entries are the incorrect identification by the computer. Overall accuracy for this classifier was $72 \%$.

\begin{tabular}{||l|l|l|l|l|l|l|r||}
\hline \hline & C1 & C2 & C3 & C4 & C5 & C6 & C7 \\
\hline C1 & 4400 & 72 & 8 & 5 & 79 & 22 & 1081 \\
\hline C2 & 96 & 3327 & 11 & 9 & 17 & 54 & 330 \\
\hline C3 & 62 & 31 & 390 & 44 & 147 & 47 & 236 \\
\hline C4 & 57 & 59 & 13 & 2100 & 47 & 267 & 390 \\
\hline C5 & 231 & 10 & 29 & 15 & 1295 & 50 & 168 \\
\hline C6 & 65 & 48 & 15 & 146 & 153 & 1146 & 137 \\
\hline C7 & 1058 & 104 & 11 & 73 & 60 & 68 & 1977 \\
\hline$P_{d}$ & 74 & 91 & 82 & 88 & 72 & 69 & 46 \\
\hline \hline
\end{tabular}

Although Culverhouse et al. [28] showed that human experts were far from perfect for certain difficult classification tasks such as plankton identification, for simplicity, we considered the human expert as a "perfect classifier" in this study. The effect of training with contaminated training samples is a very interesting research topic. Research on handwritten characters by Schölkopf \& Smola [139] suggests that classifier performance was not too sensitive to a small amount of contamination. Further study is needed to decide how "clean" the training set needs to be to have a reliable classifier(cf. classification stability, Chapter 4).

Testing the effects of different kernels and their parameters revealed that the SVM classifier was robust to both kernel function type and parameters specific to the kernel (cf. Table 5.3). For radial basis function (Gaussian kernel), the recognition rate was 
Table 5.2: Mean confusion matrix for EN302, VPR Tow 7, based on learning vector quatization method neural network classifiers built with different randomly selected sets of 200 training ROIs using hold-out method [34]. Column and row headings are as in Table 5.1. True counts (i.e. human counts) for a given taxa are given in the columns, while counts by attomatic identification (i.e. computer counts) are given in the rows. The correct identifications by the computer are given along the main diagonal, while the off-diagonal entries are the incorrect identification by the computer. Overall accuracy of this classifier was $61 \%$.

\begin{tabular}{||l|l|l|l|l|l|l|r||}
\hline \hline & C1 & C2 & C3 & C4 & C5 & C6 & C7 \\
\hline C1 & 3604 & 482 & 26 & 29 & 104 & 95 & 1048 \\
\hline C2 & 155 & 2822 & 2 & 18 & 59 & 25 & 231 \\
\hline C3 & 138 & 20 & 325 & 181 & 281 & 184 & 212 \\
\hline C4 & 127 & 26 & 26 & 1757 & 143 & 181 & 302 \\
\hline C5 & 72 & 40 & 13 & 29 & 829 & 186 & 97 \\
\hline C6 & 116 & 37 & 34 & 119 & 214 & 758 & 165 \\
\hline C7 & 1771 & 224 & 47 & 247 & 151 & 135 & 2185 \\
\hline$P_{d}$ & 60 & 77 & 68 & 73 & 46 & 48 & 51 \\
\hline \hline
\end{tabular}

not sensitive to the choice of penalty constant $C$. For the wide range of $C$ (10-500), the recognition rate only changed by $2 \%$. Recognition rate was more sensitive to the kernel width $\sigma$ for the radial basis function. However, the recognition rate was still fairly constant over a wide range of $\sigma$. For the polynomial kernel, recognition rate increased from $69 \%$ to $74 \%$ with an increase in polynomial order from 1 to 6 . For the sigmoid kernel, the change in classifier performance was relatively small, and the performance itself was similar to that obtained using the other kernels. Among all kernel methods, the top performances differed by only $1 \%$. The similarity among these different classifiers in performance improvement indicates that classification is not sensitive to the classifiers being used. Specifically, the sigmoid kernel SVM is equivalent to certain types of $\mathrm{NN}$ classfier, implying that COM features are more relevant to the plankton classification problem.

In estimating plankton abundance, the performance of COM-SVM was uniformly better than the CSF-NN classifier (Figure 5-1). Abundance estimates for both classifiers had the same trends as the hand-sorted result. Differences in abundance between these methods, quantified using the Kullback-Leibler (KL) distance method [42] for all four taxa, revealed a closer agreement between COM-SVM and hand-sorted than 
Table 5.3: Performance of the classifier with different kernel widths $(\sigma)$, regulation penalty $(\mathrm{C})$ and kernel types, where $d$ is the polynomial degree and $\kappa$ is the kernel coefficient. The recognition rate on the independent test set is shown.

Gaussian kernel $(C=50) k\left(x, x^{\prime}\right)=\exp \left(\frac{-\left\|x-x^{\prime}\right\|^{2}}{2 \sigma^{2}}\right)$
\begin{tabular}{||l|l|l|l|l|l|l|l||}
\hline \hline$\sigma$ & 0.2 & 0.5 & 1 & 2 & 3 & 5 & 10 \\
\hline Rate & 63 & 69 & 72 & 75 & 75 & 73 & 70 \\
\hline
\end{tabular}

Gaussian kernel $(\sigma=2) k\left(x, x^{\prime}\right)=\exp \left(\frac{-\left\|x-x^{\prime}\right\|^{2}}{2 \sigma^{2}}\right)$

\begin{tabular}{||l|l|l|l|l|l|r||}
\hline \hline C & 10 & 20 & 50 & 100 & 200 & 500 \\
\hline Rate & 73 & 75 & 75 & 75 & 74 & 73 \\
\hline \hline
\end{tabular}

Polynomial kernel $(C=5) k\left(x, x^{\prime}\right)=\left(<x, x^{\prime}>\right)^{d}$

\begin{tabular}{||l|l|l|l|l|l|r||}
\hline \hline d & 1 & 2 & 3 & 4 & 5 & 6 \\
\hline Rate & 74 & 74 & 74 & 72 & 69 & 69 \\
\hline \hline
\end{tabular}

Sigmoid kernel $k\left(x, x^{\prime}\right)=\tanh \left(\kappa<x, x^{\prime}>+\theta\right)$

\begin{tabular}{||l|l|l|l|l|l|r||}
\hline \hline$\kappa$ & 0.01 & 0.02 & 0.05 & 0.1 & 0.2 & 0.5 \\
\hline Rate & 69 & 71 & 73 & 74 & 72 & 60 \\
\hline \hline
\end{tabular}

between hand-sorted and CSF-NN (Table 5.4), reflecting the higher accuracy of the COM-SVM method. In order to investigate the relative contribution of COM and SVM in improving performance, the SVM classifier was trained using original features. The abundance estimation of this classifier (CSF-SVM) was compared to that of the original classifier (CSF-NN). The CSF-SVM classifier was found to perform better than the CSF-NN classifier in regions of low abundance for Chaetoceros socialis colonies. However, the CSF-SVM classifier gave underestimates in relatively high abundance regions. In overall performance, the CSF-SVM classifier and CSF-NN classifier were fairly similar (Figure 5-2). As discussed by Davis et al. [34], when the relative abundance of a taxon is above $20-25 \%$, the abundance estimation error due to misclassification falls well within the natural variation for replicate plankton tows. In areas of low relative abundance, accuracy of the abundance estimates is typically much lower $[34,146]$. The $28 \%$ reduction in recognition error results in a reduction in abundance estimate error rate for Chaetoceros socialis colonies by more than $50 \%$ in areas of low relative abundance (Figure 5-3). The reduction in abundance error rate is due to the use of both COM and SVM. Positive values indicate improved performance, while negative values indicate worse performance. COM-SVM out-performed 
CSF-SVM in most cases, except in regions of low C. socialis abundance. The latter performance difference was due to general underestimation by the CSF-SVM classifier and consequent increase in $C$. socialis abundance in these regions. These observations further support the idea that use of texture-based features (i.e. co-occurrence matrix) is the main reason for performance improvement in our classification system.
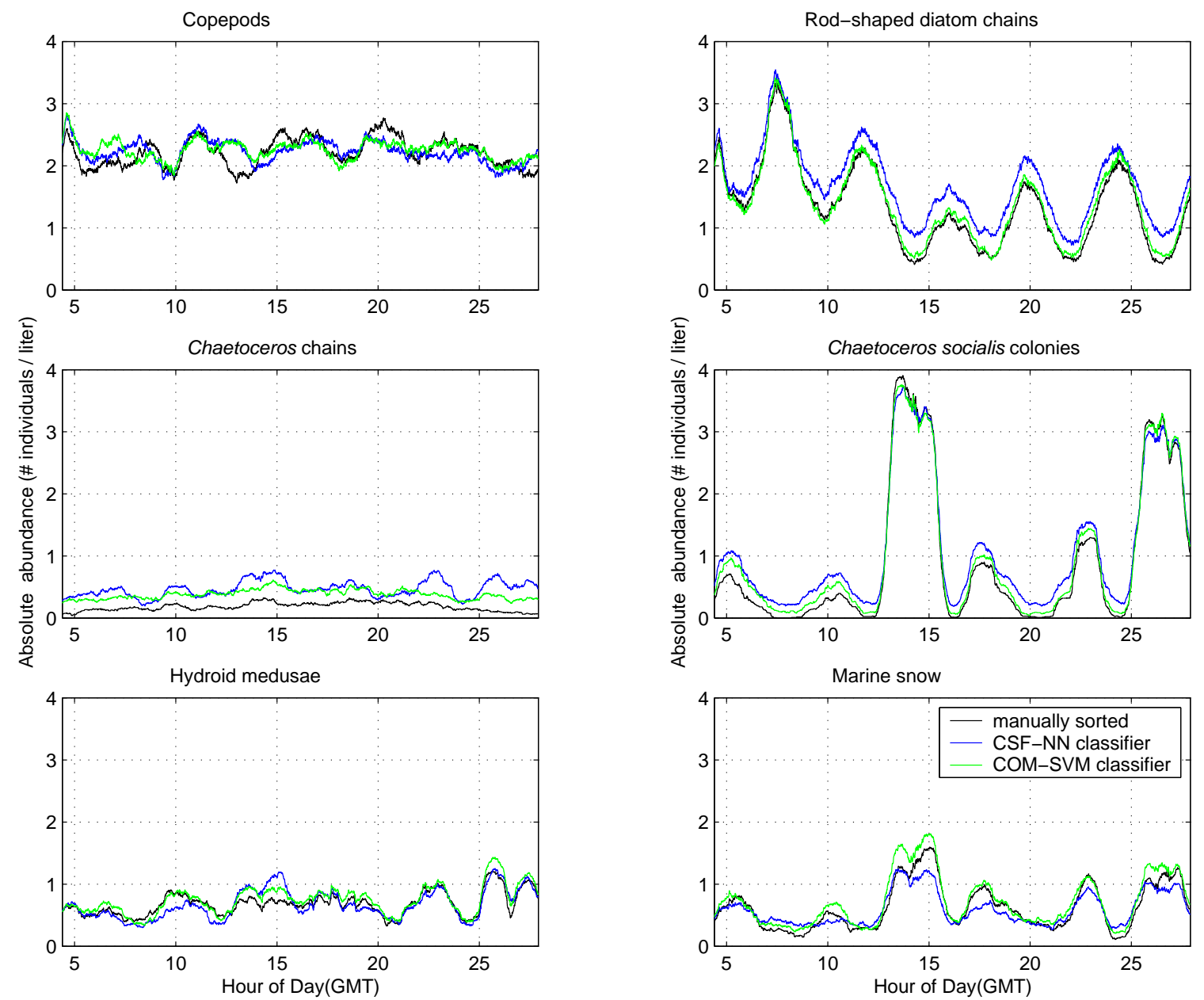

Figure 5-1: Comparison of 2 automated classifier with human expert classified results for 6 dominant taxa along the tow timescale. CSF-NN, combined shape-based features and neural network; COM-SVM, co-occurrence features and support vector machine. The data are first binned into 10 second time intervals. A 1 hour smoothing window is applied to the binned data.

The pair-wise approach was chosen in order to extend the binary SVM classifier to the multi-class SVM classifier used in this study. Another approach using the Error-Correcting Output Coding method [39] also appears to be very promising and 

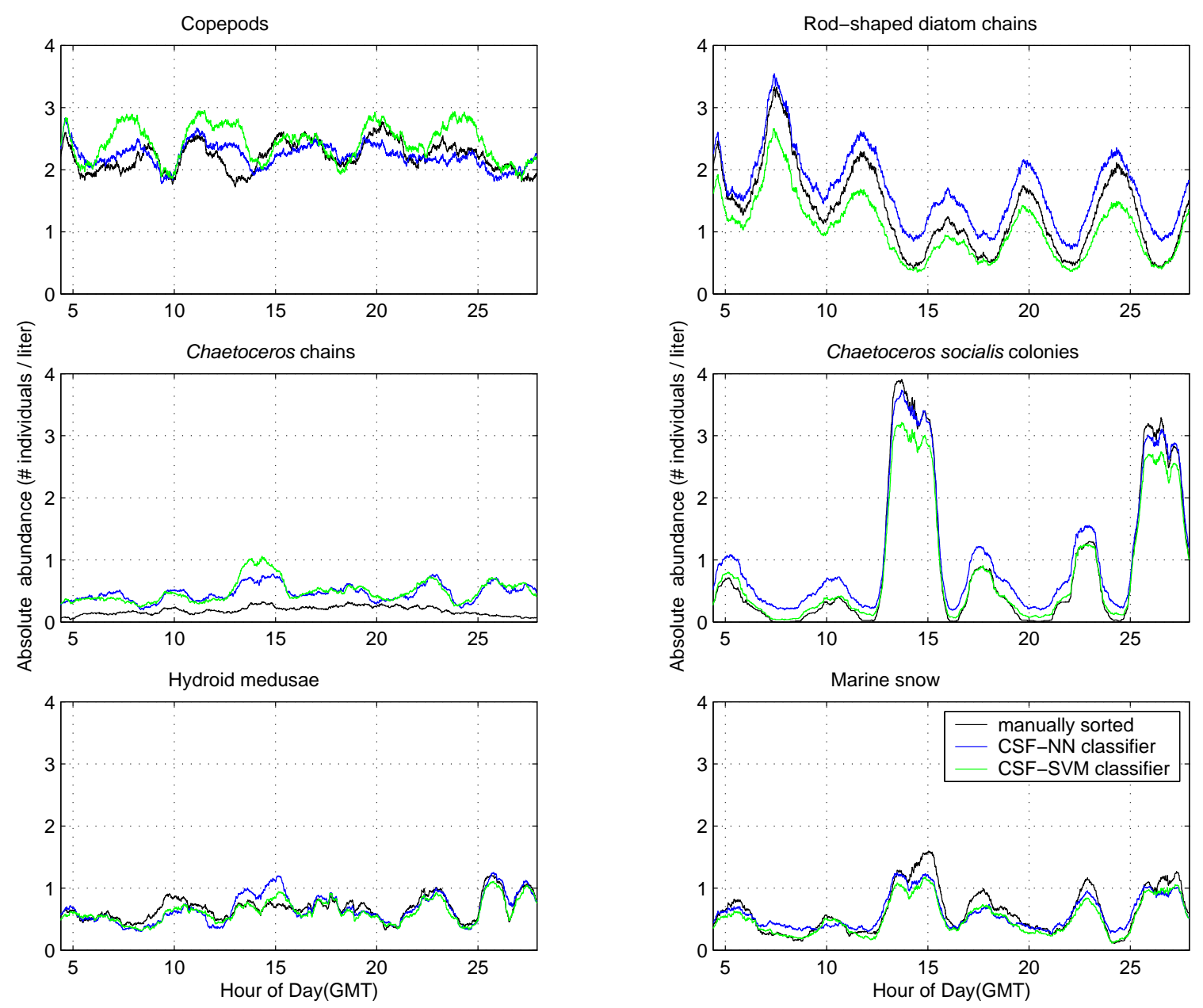

Figure 5-2: Comparison of 2 automated classifier with human expert classified results for 6 dominant taxa along the tow timescale. CSF-NN, combined shape-based features and neural network; CSF-SVM, combined shape-based featuresand support vector machine. The data are first binned into 10 second time intervals. A 1 hour smoothing window is applied to the binned data.

Table 5.4: Kullback-Leibler(KL) distance estimation for difference in abundance between COM-SVM and hand-sorted and between CSF-NN and hand-sorted. Row headings are as in Table 5.1. The KL distance is dimensionless. For two identical abundance curves, the KL distance is 0 , while for two random distributions, the KL distance is 0.5. Note lower values of COM-SVM than CSF-NN for all four taxa.

\begin{tabular}{||l|l|l|l|l|l|r||}
\hline \hline & C1 & C2 & C3 & C4 & C5 & C6 \\
\hline$D_{K L}(C O M-S V M$, hand $)$ & 0.0036 & 0.0022 & 0.0302 & 0.0225 & 0.0048 & 0.0075 \\
\hline$D_{K L}(C S F-N N$, hand $)$ & 0.0041 & 0.0113 & 0.0757 & 0.0742 & 0.0188 & 0.0279 \\
\hline \hline
\end{tabular}



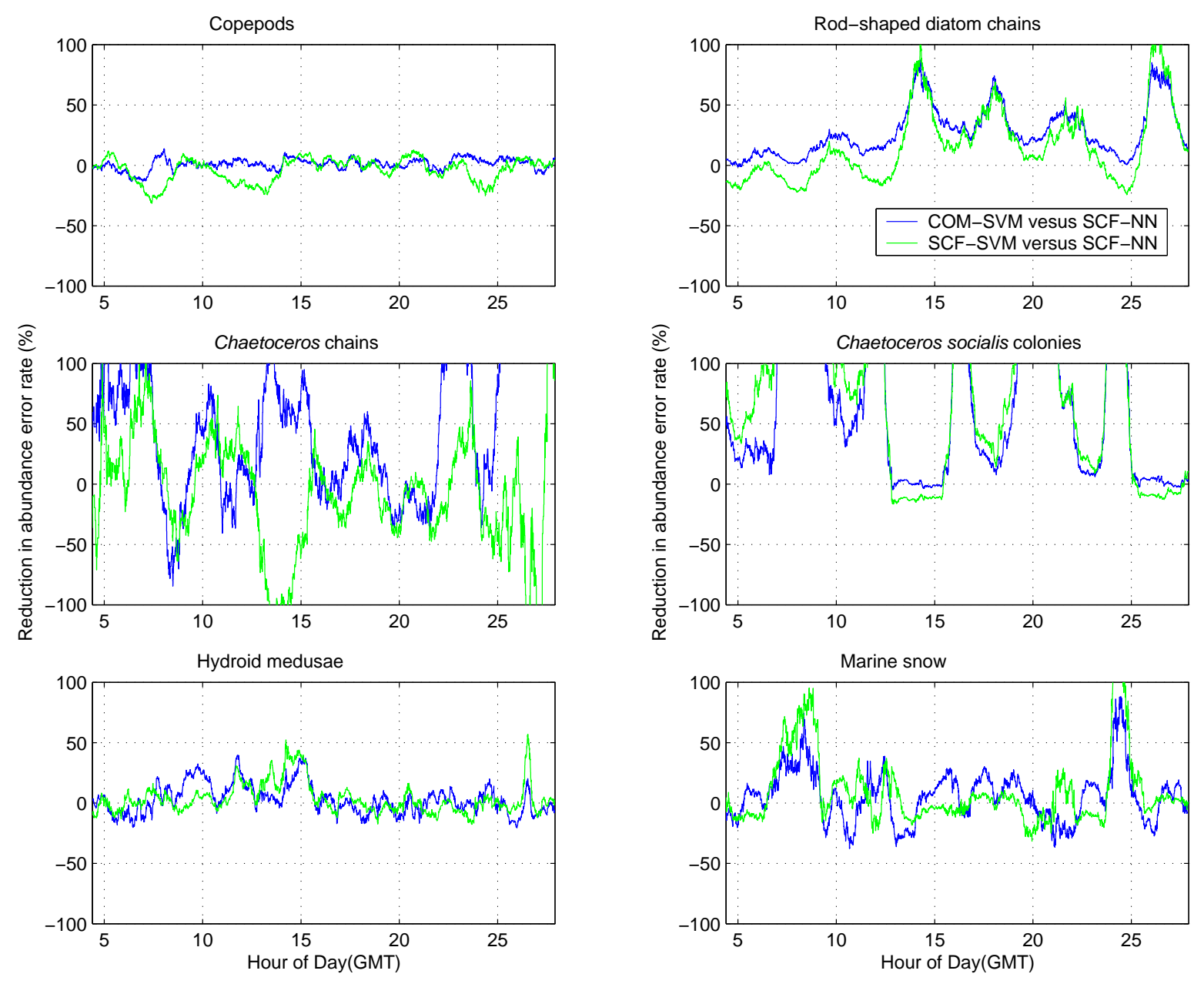

Figure 5-3: Reduction in the relative abundance estimation error rate between COMSVM and CSF-NN, and between CSF-SVM and CSF-NN. The positive value indicates that COM-SVM/CSF-SVM is better than CSF-NN, while the negative value indicates COM-SVM/CSF-NN is worse than CSF-NN. 
is becoming an active research topic [3, 26, 122]. Further analysis of this method is the subject of future study.

The new COM-SVM method only uses texture-based features (i.e. co-occurrence matrices) to automatically classify plankton images. Shape-based features also carry a substantial amount of information that can be used for classification. An attempt was made to directly stack texture-based features and shape-based features into a single feature vector, and train the classifier on this single feature vector (with and without principal component analysis). Only a very limited improvement (less than $1 \%$ ) in recognition rate was obtained. This method of combining features was only one approach, and further research is needed to determine whether other methods (such as weighting each individual feature by its discriminative power) for combining features may yield improved identification accuracy. Given the growing trend toward optical imaging of marine biota, new methods of automatic identification are needed to improve classification accuracy. The texture-based method presented here can be used for a wide-variety of image classification problems, since it is not sensitive to occlusion and lighting gradients and is independent of shape-based features.

\subsection{Conclusion}

In this chapter, I have used texture-based feature, co-occurrence matrices, to classify plankton images taken in the field using the VPR. This method had $72 \%$ overall recognition rate compared to $61 \%$ for a previous recognition system that used shapedbased features. Shape-based features are the primary ones currently used in automatic plankton recognition systems due to their early success on plankton images taken in the laboratory. Texture-based features have been found to work better for fieldcollected images of plankton because they are less sensitive to occlusion, non-uniform lighting, and projection variance.

SVM was used to train the classifier. Classifier performance was not sensitive to kernel type or to the exact parameter values used for specific kernels. In Chapter 3 , we know that selection of representative training samples is an important factor. 
In order to accurately assess classifier performance, a random set of training samples from the field is recommended.

Multi-scale texture features are captured with multiple separation distances. Scale invariance is achieved by normalization of co-occurrence matrices. Rotation invariance is achieved by using only the range and mean co-occurrence matrices.

Continued improvements in accuracy of automatic image recognition methods will enable wider use of this powerful approach. The growing use of underwater optical imaging methods requires more emphasis on development and improvement of new automatic identification techniques.

The method described here is a step toward the long term goal of highly-accurate automatic identification of plankton from optical imaging systems. 


\section{Chapter 6}

\section{Dual classification system and accurate plankton abundance estimation}

In the previous chapter, I demonstrated that using features from multi-scale cooccurrence matrices can improve the plankton classification significantly. The automatic classification results in general yield very good agreement with those obtained with manually sorted results. However, in regions of relative low abundance or for a taxon with relative low abundance, the classification is not accurate enough to estimate taxonomic group abundance. In this chapter, I have developed a dual classification method to cope with these two situations. The dual-classification system developed a rejection metric obtained by voting with 2 classifiers: 1) an NN classifier built from shape-based features and 2) an SVM classifier built from texture-based features. Both classifiers must agree on the identification of an image for it to be considered true, otherwise it is classified as "unknown". Abundance estimation from the dual-classification system was corrected based on detection and false-alarm rates. After correction, the abundance estimation from the automatic classification system agreed very well with that derived from manually sorted results. This chapter was published in Marine Ecology Progress Series[74].

This chapter is organized as follows. The dual-classification system is described in 
Section 6.1. The dual-classification results are compared against single classification results in 6.2. A short conclusion is made in Section 6.3

\subsection{Dual classification system description}

\subsubsection{Pattern representations}

Five different types of features have been used in the dual-classification system, including shape-based features (moment invariants, morphological measurements, Fourier descriptor and granulometry curves) and texture-based features (co-occurrence matrix).

\section{Moment invariants}

Moment invariants, introduced by $\mathrm{Hu}$ [72], are based on normalized central moments, and are translation, rotation, and scale invariant. They have been widely used in plankton identification $[82,81,150,149,34,106]$.

\section{Morphological measurements}

Jeffries et al. [82, 81] first used 7 morphological measurements as features to identify zooplankton. The concept of using morphological measurement as features in plankton recognition has been commonly accepted ever since then $[34,106]$. In this chapter, 6 morphological measurements were used as part of the shape-based feature set: 1) a shape factor based on the perimeter and area of the object; 2) a ratio of maximum and minimum principal moments of the object; 3) a ratio of longest and shortest dimensions of the bounding box surrounding the object; 4) a ratio of the width at center of the object to shortest dimension of the bounding box; 5) a ratio of the left 1/4-width of the object to shortest dimension of the bounding box of an object; 6) a ratio of the right $1 / 4$-width of the object to shortest dimension of the bounding box [34]. 


\section{Fourier descriptors}

Fourier descriptors (FD) are well-studied invariant features used to describe the contour of an object. Depending on what functions are used to describe the contour, FDs can be grouped into 3 major categories, namely tangent-angle FDs [175], complex FDs [57], and elliptic FDs [94]. FDs are the Fourier coefficients when the contour function is approximated by a Fourier series. Normalized FDs were used as features to classify plankton images $[82,81]$. In this study, I used a centroidal radius-vector function (distances from the centroid to perimeter pixels) as the contour model ${ }^{1}$. The first 64 elements of the normalized power spectrum, obtained from the Fourier transform of centroidal radius-vector function were used as a feature set [150, 149, 34]. These elements were also translation, rotation, and scale invariant.

\section{Granulometry}

The concept of granulometry was introduced by Matheron [112] to study size distribution of binary images. The operation involves a series of openings/closings with structuring elements of increasing/decreasing size [140]. Tang et al. [150] first used granulometry features to classify plankton images. They found that the granulometry was more powerful in discriminating plankton images than common moment invariants and Fourier descriptors. However, these operators are computationally expensive. Fast algorithms $[162,114]$ were developed for very limited structural elements. In this chapter, Vincent's algorithm [162] was used to calculate the linear opening and closing spectra, as well as pseudo-opening and -closing spectra. Each spectrum has 40 elements, resulting in 160 features for granulometry.

\section{Co-occurrence matrix}

Gray level co-occurrence matrices(GLCM) were first proposed by Haralick et al. [59] as a texture feature to classify satellite images. It is based on estimation of the joint

\footnotetext{
${ }^{1}$ As discussed in Chapter 4, radius-vector functions are only suitable for star-shaped contour models. Most plankton images are not star-shaped. As shown in Chapter 4, the difference between different contour models are very small. To be consistent with earlier works, radius-vector functions were used in this study.
} 
probability distribution of pairs of pixels with gray-scale level $i$ and $j$, spatial distance $d$, and angle $\theta$ in an image. Hu \& Davis [73] first used GLCM to classify plankton images. They concluded that these texture-based features were more useful for classifying field-collected plankton images, due to occlusion, non-linear illumination and projection variance of the images.

\subsubsection{Feature extraction}

\section{Shape-based features}

All the shaped-based features were stacked into 1 feature vector. The features included 7 moment invariants, 6 morphological measurements, 64 Fourier descriptor coefficients, and 160 granulometry measurements [34]. Each feature element was normalized to have zero mean and unit standard deviation. Principal component analysis was applied on the feature vector to calculate dominant eigenvectors. The first 30 features associated with the largest eigenvalues were saved as the feature vector, and corresponding feature vectors were saved as a transformation matrix [150].

\section{Texture-based features}

Four different distance $(1,4,8,16$ pixels $)$ pairs and four different angles $\left(0,45,90,135^{\circ}\right)$ were used to generate co-occurrence matrices. For each separation distance, there were 4 co-occurrence matrices from 4 different angles. Only the mean and range of these matrices were used to achieve relative rotation invariance. Normalization was also applied to the resulting matrices to achieve scale invariance. The angular second moment (energy), contrast, correction, variance, inverse-difference moment, entropy, sum entropy, and difference entropy of these matrices [59, 73] were calculated and used as feature vector elements. Each feature element was further normalized to have zero mean and unit standard deviation. For each image, 64 features were used [73]. 


\subsubsection{Classifiers}

The learning vector quantization neural network classifier and support vector machine classifier were used in this study.

\section{Learning vector quantization}

Learning vector quantization (LVQ) is a supervised version of vector quantization. Its objective is to learn a set of prototypes (codebooks) which best represent each class. We implemented it with an artificial neural network $[149,34]$. LVQ neural network (LVQ-NN) is a method to divide n-dimensional feature space into different taxonomic regions by fitting neurons to the training data. The neural network has 2 layers, namely a competitive layer and a linear output layer. The complexity of the neural network (prototypes of subclass, number of neurons) was based on the number of training samples and the number of classes in the classifier. For the 200 samples per taxon, I used 20 neurons per taxon for the competitive layer. The number of outputlayer neurons was equal to the number of taxa. The weights of the neurons for each class were initialized to the mean of the training feature vectors for that class plus a small random value. The network was trained by randomly presenting the training samples to the network. For a given training sample, the nearest neuron (winning neuron) was found (i.e. shortest Euclidean distance between the training samples to all the neurons in feature space). The taxon assigned to this nearest neuron was the "predicted" taxon of the neuron network. If the prediction was correct, the weights of this winning neuron (prototype) were updated in such a way to move that neuron a step closer to the training sample in the feature space. Otherwise, the weights of the winning neuron were updated such that it was pushed a step away from that sample in the feature space. The learning rate (step size) was preset from the trade-off between the training time and the training error. A small learning rate was usually associated with long training time and small training error, while a large learning rate was usually associated with short training time and big training error. Over-training was avoided by using number of neurons and epoches established in Chapter 3 . 


\section{Support vector machine}

The support vector machine (SVM) is a margin-based linear machine. It was first proposed by Vapnik [160, 161]. Instead of using neurons, the basic idea of SVM is to find a hyperplane which separates the training samples with maximum margin. The capacity of linear SVM is often limited. In order to deal with the non-linear problem, an intermediate step is taken to map original features to a much higher dimensional space; a hyperplane is then constructed on that high space. The mapping step is usually time consuming. The trick of nonlinear SVM is to pick certain mapping functions which satisfy Mercer's condition so that the mapping is equivalent to applying a kernel function on the original features. In these cases, the mapping is not necessary. Nonlinear SVM is solved exactly like linear SVM, except the original feature vector is replaced by a kernel function of the feature vector. SVM is closely related to structural risk minimization and regularization theory. It has shown a nice gener-

alization property and resistance to over-training in a number of real-world problems $[118,37,92,117,73]$. SVM is primarily a binary classifier. Three approaches are often used to extend SVM to multi-class case, namely one-vs-all approach, pairwise approach, and error-correcting output codes approach. In the last chapter, I showed that the SVM classifier was not very sensitive to kernel types and kernel parameters. In this chapter, I chose a linear kernel function to avoid extra labeled validation samples which were needed in kernel parameter selection. The pairwise approach was used, since it yielded balanced training in this case [73].

\subsubsection{Dual classification system}

The schematic diagram of the dual classification system is shown in Figure 6-1. During the training phase, two classifiers were built in parallel. An LVQ-NN classifier was built from shape-based features as discussed in the feature extraction section. At the same time, an SVM classifier was built using texture-based features from the same training samples. In the classification phase, shape-based and texture-based features were calculated from all the samples. An LVQ-NN classifier made the identification 
based on shape-based features, while an SVM classifier made the identification based on texture-based features. In the end, a classifier committee was called. If the labels predicted by the two classifiers belonged to the same class, the sample was labeled as that class. Otherwise, the sample was labeled as "unknown".

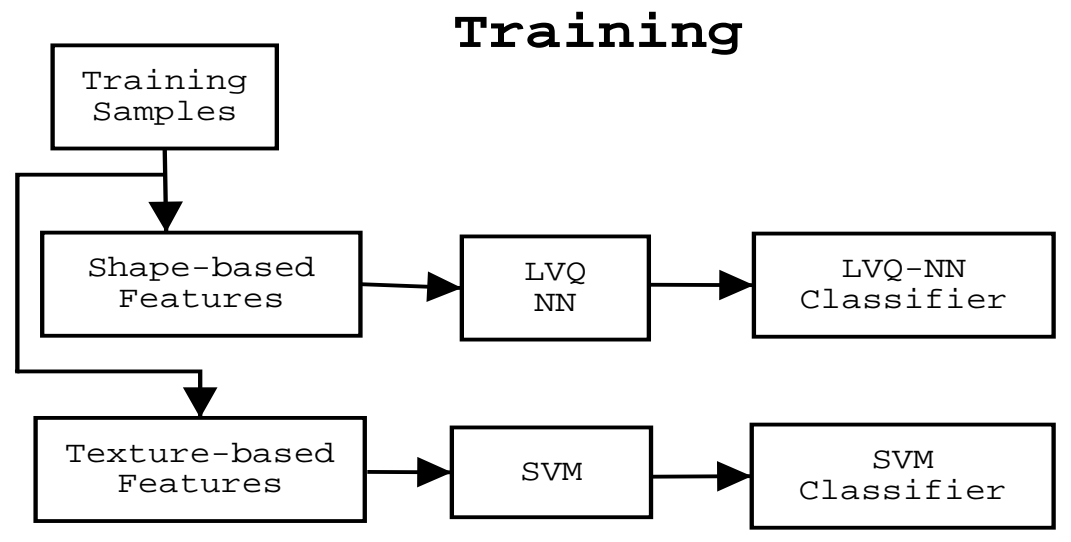

\section{Classification}

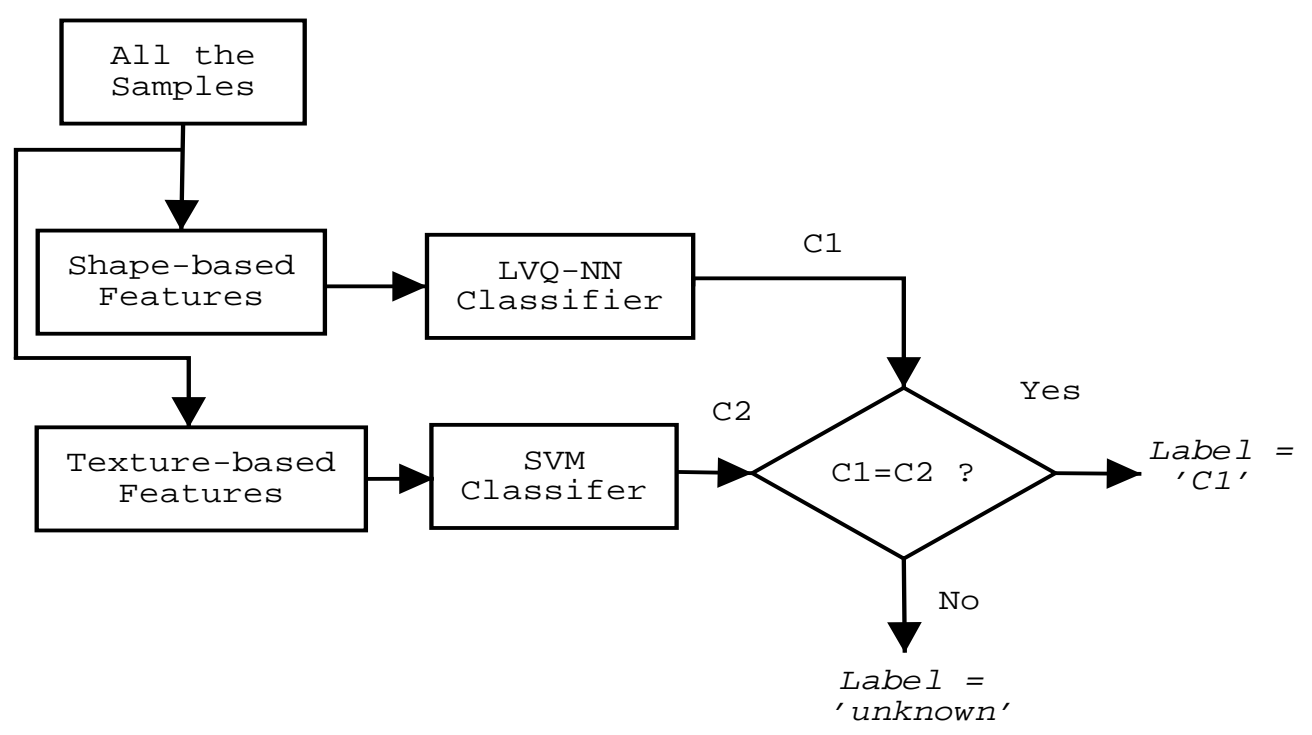

Figure 6-1: Schematic diagram of dual-classification system. LVQ: learning vector quantization; NN: neural netowork; SVM: support vector machine 


\subsubsection{Classification performance evaluation and abundance correction}

\section{Confusion matrix}

The confusion matrix is used to assess the accuracy of automatic classification. The number of images manually sorted by a human is given in the columns ( 1 column per taxon), while the number of images automatically classified by a computer is given in the rows (1 row per taxon). Diagonal elements correspond to agreement between human and machine. In this chapter, the confusion matrix of the dualclassification system was built in the following way. First, a $7 \times 6$ matrix was built from a set of training images (200 per taxon) for 6 dominant taxa (Table 6.1) using the leave-one-out method (cross-validation) [34]. The 7th row in this matrix contains the "unknown" counts. Second, 200 images that had been manually sorted into an "other" category were classified using the dual-classification system to fill in the 7th column ${ }^{2}$. The resulting $7 \times 7$ matrix was used as the confusion matrix for the dualclassification system (i.e. Table 6.1).

From the matrix, some simple indexes of classifier performance can be calculated. The most used indexes are probability of detection (also known as sensitivity or probability of true positives), and probability of false alarm (also known as probability of false positives). The probability of detection, $P_{D}$, measures the probability that the classification system will label correctly for each class given the object belongs to that class, i.e. $P_{D}=$ true positive counts/(true positive counts + false negative counts). The probability of false alarm is the probability that an image will be classified as a given taxon when it does not actually belong to that taxon. Another related concept is specificity, $\mathrm{SP}[6]$, which is the probability that a classifier's prediction is correct for each taxon, i.e. $\mathrm{SP}=$ true positive counts $/$ (true positive counts + false positive counts). The probability of detection and specificity of each taxon were calculated from the confusion matrix to correct the abundance estimation.

\footnotetext{
${ }^{2}$ The last classifier built in leave-one-out method was used.
} 
Table 6.1: Confusion matrix of the dual-classification system, using the leave-one-out method. Randomly selected images (200 per category) from EN302 VPR tow 7 were used to build the confusion matrix. C1: copepods, C2: rod-shaped diatom chains, C3: Chaetoceros chains, C4: Chaetoceros socialis colonies, C5: hydroid medusae, C6: marine snow, $\mathrm{C} 7$ : other, $\mathrm{C} 7$ : unknown, $P_{D}$ : probability of detection $(\%), \mathrm{SP}=$ specificity (\%). NA: not applicable. True counts (i.e. human counts) for a given taxa are given in the columns, while counts by classification system are given in the rows. Correct identifications by the computer are given along the main diagonal, while the off-diagonal entries are the incorrect identification by the computer. All data are counts, except in the last row and last column, which are percent values. Although images from the "other" category are not needed to train the dual-classification system, they are necessary to evaluate it.

\begin{tabular}{||l|l|l|l|l|l|l|l|r||}
\hline \hline & C1 & C2 & C3 & C4 & C5 & C6 & C7 & SP \\
\hline C1 & 146 & 0 & 3 & 2 & 1 & 2 & 59 & 69 \\
\hline C2 & 2 & 176 & 2 & 0 & 1 & 1 & 13 & 90 \\
\hline C3 & 0 & 0 & 122 & 1 & 3 & 1 & 2 & 95 \\
\hline C4 & 0 & 0 & 0 & 145 & 2 & 8 & 12 & 87 \\
\hline C5 & 0 & 0 & 5 & 0 & 111 & 6 & 4 & 88 \\
\hline C6 & 1 & 0 & 0 & 3 & 4 & 98 & 4 & 89 \\
\hline C7 & 51 & 24 & 68 & 49 & 78 & 84 & 106 & 23 \\
\hline PD & 73 & 88 & 61 & 73 & 56 & 49 & 53 & NA \\
\hline \hline
\end{tabular}




\section{Abundance correction}

If $P_{D}$ and $\mathrm{SP}$ of a classification system for each taxon are always the same, plankton abundance estimated from the classification system will be perfect although the classfication system itself is not perfect. In reality, $P_{D}$ and specificity may change for different-sized evaluation data sets. In particular, the specificity of a taxon is positively related to the relative abundance of that taxon. However, if the variation in $P_{D}$ and $\mathrm{SP}$ of a classification system for each taxon is relatively small in the study area, we can automatically correct the abundance estimation from the classification system using the following steps: 1) estimate $P_{D}$ and SP for each taxon from the confusion matrix; 2) scale the abundance estimation from the classification system for each taxon by the ratio $S P / P_{D}$ for that taxon. The manual correction method discussed in Davis et al. [34], involves removing all false alarms manually from the classification results. In that case, the specificity of each taxon was unity, and the correction factor for each taxon was $1 / P_{D}$. This correction method is different from the statistical correction method discussed in Chapter 3 [146].

\subsection{Classification results}

The first 25 images classified as copepods and Chaetoceros socialis colonies by the dual classification system and by the single neural network classifier [34] are shown in Figure 6-2. For the taxa with high relative abundance (i.e. copepods), the performance of dual-classification and single classifier is very similar, which implies the two classification systems have very close probability of detection. On the other hand, for taxa with lower relative abundance (i.e. C. socialis), the dual classification system has a far lower false alarm rate (Figure 6-2). The dual classification system has much higher specificity for C. socialis in regions of low relative abundance (cf. Table 6.1, 6.2). In other words, the dual classification system makes the specificity less variable with changes in relative abundance of a taxon, which makes automatic correction of classification results possible (Tables 6.1, and 6.2).

Abundance estimation of 6 dominant taxa were compared between manually 
A
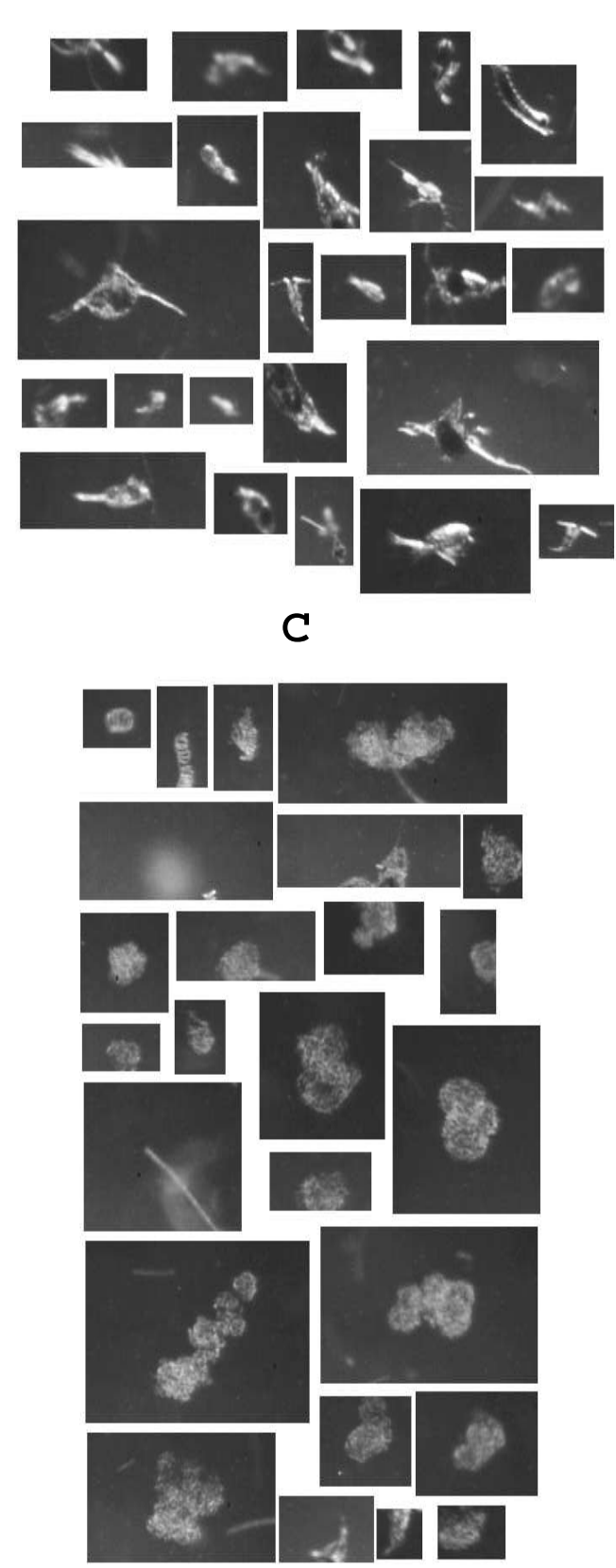

B

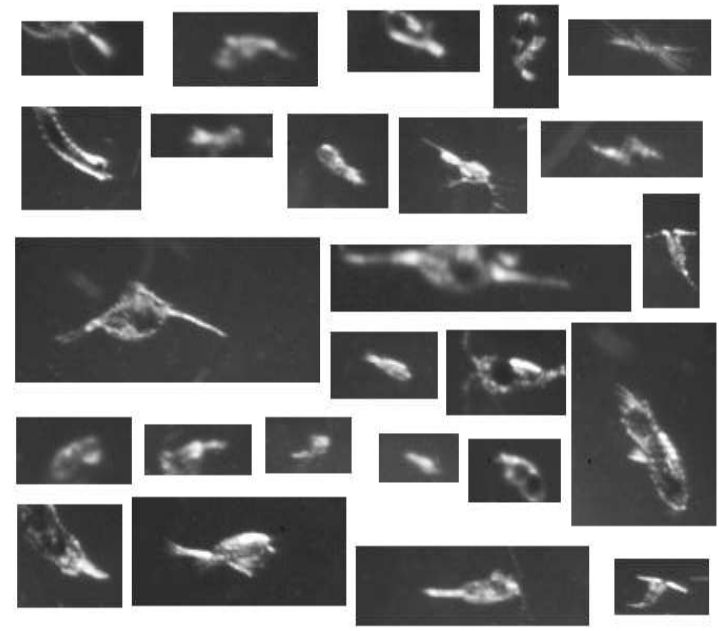

D

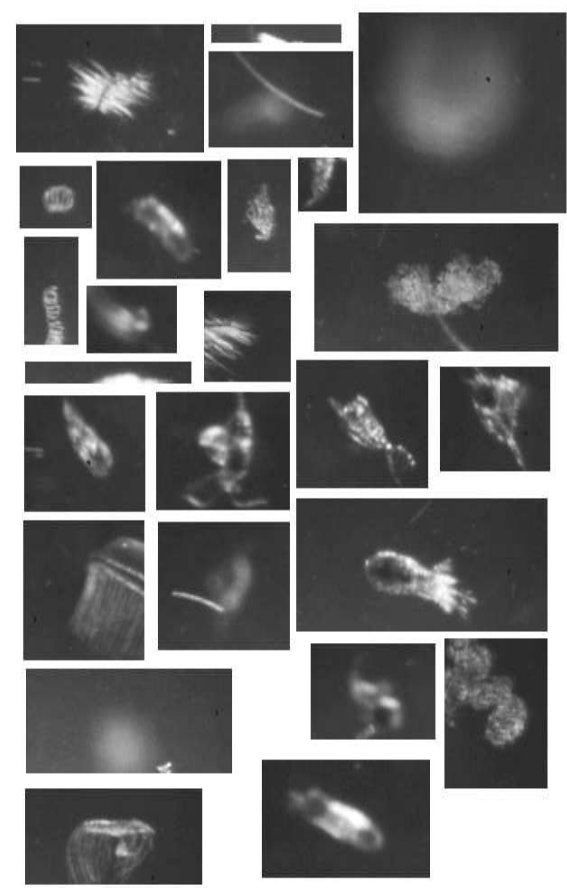

Figure 6-2: Automatically classified images: comparison of results for $(A, C)$ dualclassification system and (B,D) single neural network classifier. The first 25 images classified as (A,B) copepods and (C,D) Chaetoceros socialis by the dual-classification system and LVQ-NN classifier are shown. For taxa having relatively high abundance, such as copepods, both systems yield very similar results (21 out of 25 were the same). In contrast, for taxa having relatively low abundance, such as low-abundance regions of C. socialis, the dual-classification system has much higher specificity (fewer false alarms). 
Table 6.2: Confusion matrix of the single LVQ-NN classifier, using the leave-oneout method. Images used were the same as those in Table 6.1. Abbreviations as in Table 6.1. All data are counts, except in the last row and last column, which are percent values.

\begin{tabular}{||l|l|l|l|l|l|l|l|r||}
\hline \hline & C1 & C2 & C3 & C4 & C5 & C6 & C7 & SP \\
\hline C1 & 132 & 8 & 16 & 2 & 16 & 7 & 54 & 56 \\
\hline C2 & 6 & 171 & 1 & 4 & 7 & 5 & 20 & 80 \\
\hline C3 & 7 & 1 & 123 & 10 & 23 & 22 & 11 & 62 \\
\hline C4 & 6 & 2 & 14 & 153 & 5 & 20 & 14 & 72 \\
\hline C5 & 5 & 5 & 13 & 4 & 105 & 21 & 9 & 65 \\
\hline C6 & 9 & 3 & 20 & 17 & 34 & 109 & 15 & 53 \\
\hline C7 & 35 & 10 & 13 & 10 & 10 & 16 & 77 & 45 \\
\hline PD & 66 & 86 & 62 & 77 & 53 & 55 & 39 & NA \\
\hline \hline
\end{tabular}

sorted, dual classification with automatic correction, single NN classifier of original feature with manual correction [34](Figure 6-3). The manual correction method [34] requires manual removal of false negative images from the automatically classified results for each taxon. The result was then further corrected by the probability of detection which was estimated from Table 6.2. The automatic correction method estimated probability of detection and specificity for each taxon from the confusion matrix (Table 6.1), and used the correction factor discussed in the section 6.1.5.

Except for the copepod category, the manually sorted, manually corrected and dual classification curves lie almost on top of each other (Figure 6-3). The high agreement between manually corrected and manually sorted results for copepods is due to the incorrect assumption that the human-sorted results were perfect and invariant. For this case, false negative samples were determined using a lookup table from manually sorted images (i.e. no variations between manually corrected and manually sorted results) rather than by manually correcting the classification result as discussed by Davis et al.[34]. The high agreement between manually sorted and manually corrected results of copepods abundance is an artifact of such a treatment. In fact, among the manually sorted images, there is some overlap between copepods and the "other" category due to ambiguity in appearance of some of the "other" images, which may actually have been copepods oriented in such a way as to make 

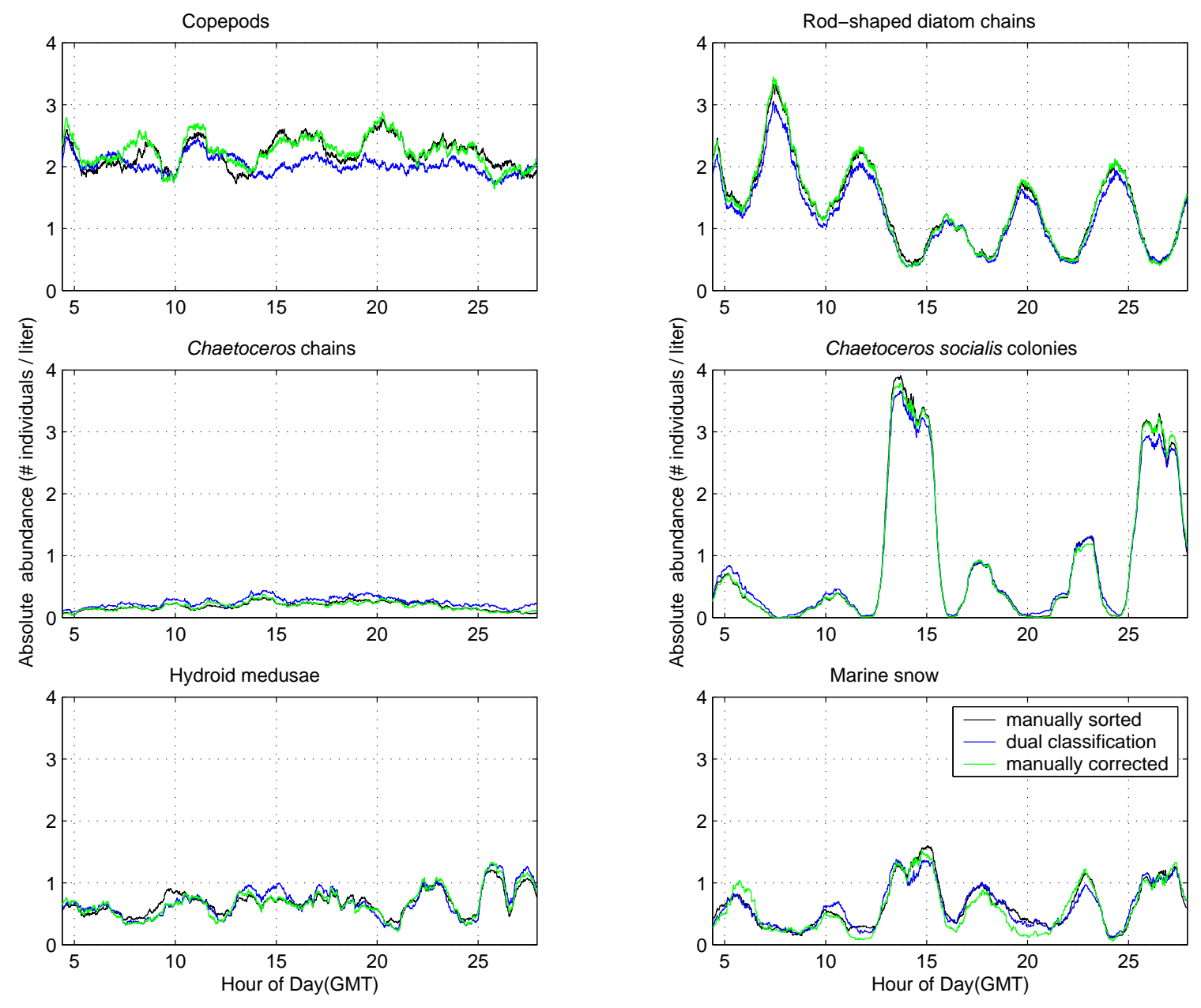

Figure 6-3: Comparison of dual-classification, and manually corrected single NN classification with human expert classified results for 6 dominant taxa along the tow timescale. The data are first binned into 10 second time intervals. A 1 hour smoothing window is applied to the binned data. 
identification by a human difficult.

According to the study by Culverhouse et al. [28], trained personnel can be expected to achieve 67 to $83 \%$ self-consistency in difficult labeling tasks. Our copepods category should belong to this case. That is to say, if a human labels all 20,000+ images a second time, the copepod abundance estimation between the two human results is likely to differ. The mean abundance estimation for copepods between automatically classified and manually sorted results is very close. The uncertainty in the manually sorted abundance estimation is comparable to the abundance difference between automatic and manually sorted results.

Abundance estimation of 6 dominant taxa were compared between 3 automatic classifiers (dual-classification, single NN classifier without correction, SVM classifier from co-occurrence feature) and manually sorted results (Figure 6-4). For taxa in high relative abundance regions, the 3 automatic classification systems agree very well with manually sorted results. However, for taxa having low relative abundance or taxa having low relative abundance regions, the reduction of the abundance error rate is marked (Figure 6-4). Chaetoceros chains make up less than $2.5 \%$ of total plankton in this tow. The abundance estimation error of the dual-classification system is uniformly less than $50 \%$ along the tow path, which is smaller than the natural variation for replicate plankton tows $[171,34]$. In the regions of extremely low relative abundance (e.g. Figure 6-3, hour 8 and 12, Chaetoceros socialis colonies), the dual classification system estimates the abundance significantly higher than manually sorted or manually corrected abundance.

The reduction in abundance error rates of the dual classification system compared to the single NN classifier [34], the SVM classifier with co-occurrence matrices [73], and manual correction [34] are given in Figure 6-5. For copepods, the manually corrected result outperforms other methods. As discussed above, this difference is not significant, due to low confidence of the manually sorted result. For rodshaped diatom chains, the performances of dual classification, manually corrected, and COM-SVM are very similar. They all outperform the single NN classifier. Dual classification has a significant reduction in abundance error compared to OF-NN and 

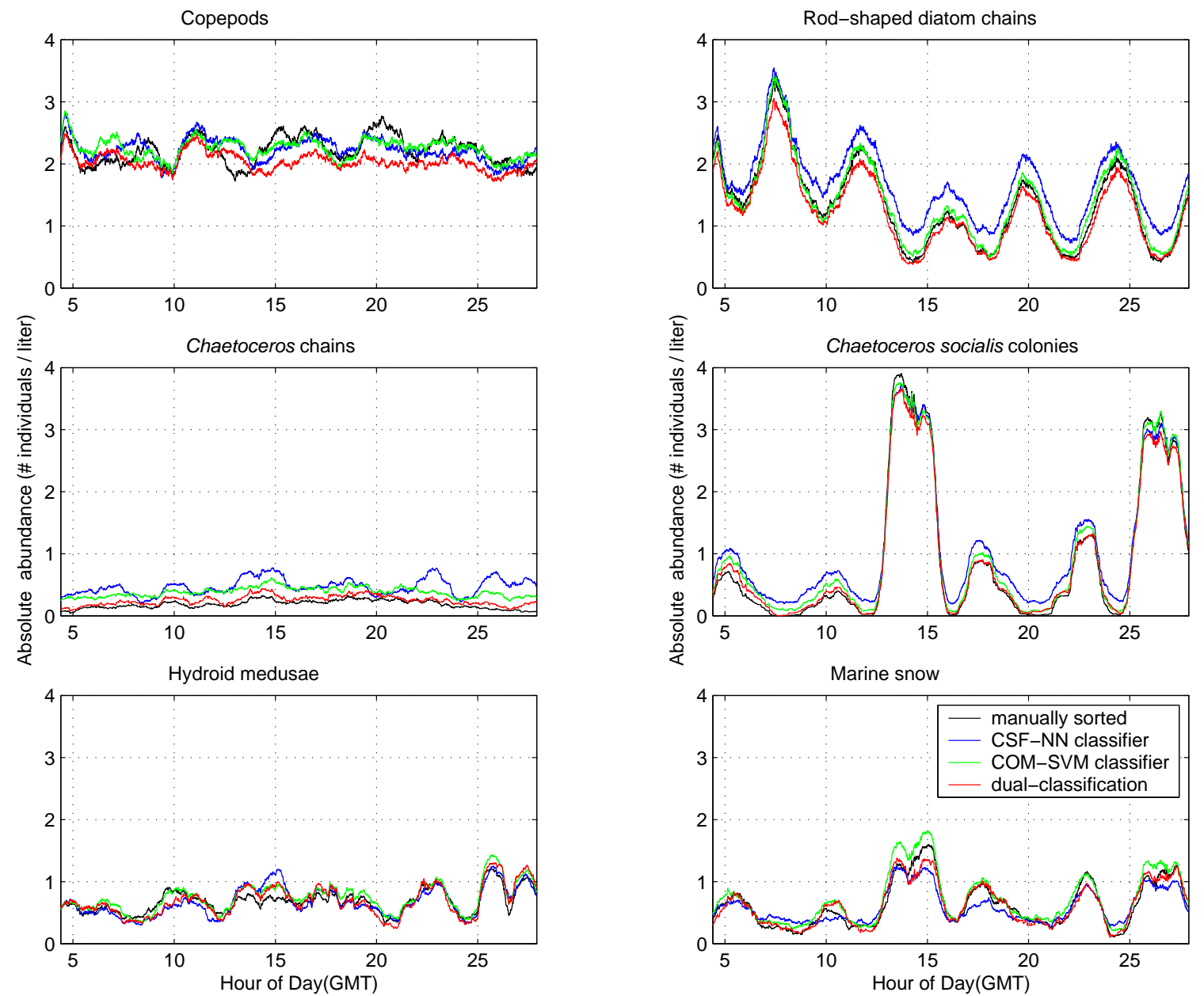

Figure 6-4: Comparison of 3 automated classifier with human expert classified results for 6 dominant taxa along the tow timescale. CSF-NN, combined shape-based features and neural network; CSF-SVM, combined shape-based features and support vector machine. The data are first binned into 10 second time intervals. A 1 hour smoothing window is applied to the binned data. 
COM-SVM, while it is close to the manually corrected results. It is the same case for C. socialis colonies. The performance disagreement occurs in the regions of extremely low relative abundance. As discussed by Benfield et al. [9], these regions could be the limits of the optical sampling method (i.e., high magnification VPR camera used). The performance of four different methods on hydroid medusae and marine snow is very close. The dual classification method performs slightly better for marine snow, while the manually corrected method is better for hydroid medusae.

The advantage of using the dual-classification system is to reduce the false alarm rate of each taxon to such a low level that the variation of specificity for each taxon is low in the whole study region (Figure 6-6). This makes fully automatic correction possible. The dual-classification system substantially decreases the probability of false alarm, while only slightly reducing the probability of detection. By rejecting a small portion of the images as "unknown", identifications are made by the dualclassification system with higher confidence. Thus, it is not necessary to classify all the images into taxonomic groups to achieve better abundance estimation.

In this chapter, I present one way to integrate shape-based features with texturebased features. Other approaches to incorporate shape-based features to texturebased features are certainly possbile. In the simplest example, all available features are stacked into 1 feature vector and used in training an SVM or LVQ-NN classifier as I did for combining shape-based features. I have found that such an approach is not efficient, and that the result is almost identical to the COM-SVM method. I have also tried more sophisticated approaches to reduce feature dimension without losing discriminant power, but have thus far met with little success. Such approaches require further research.

A dual-classification system utilizes a greater range of variation in feature sets and classifiers. The second classifier provides additional information that the first classifier alone does not possess. It is certainly possible to use 1 type of classifier (e.g. SVM or LVQ-NN) with all types of features or 1 type of feature for both classifiers. However, the variability gained by the dual-classification system using different features and different classifers would be reduced. 


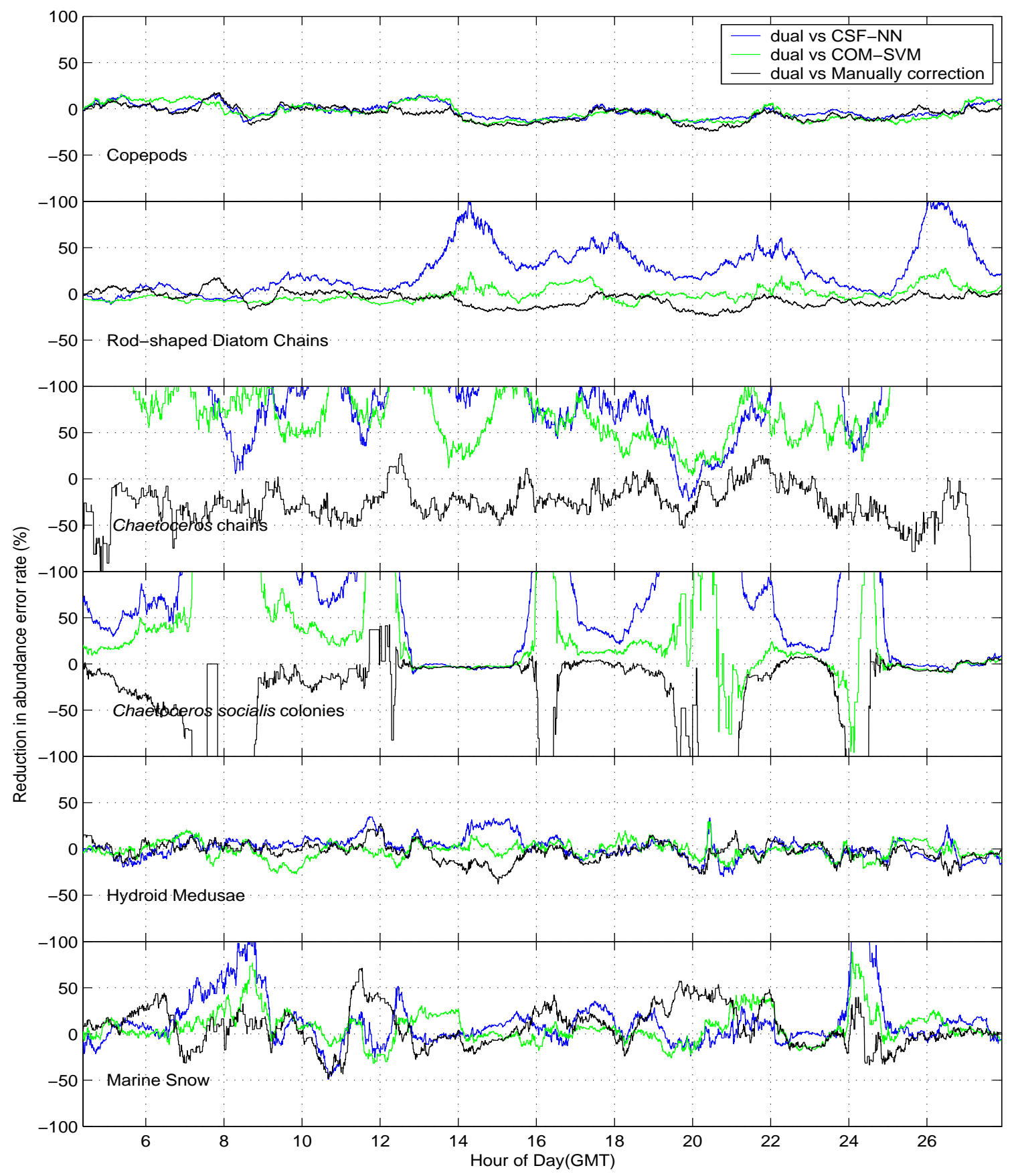

Figure 6-5: Reduction in error rate between the dual- and single-classification systems for relative abundance estimation. Positive values indicate that the dual-classification outperforms other methods; negative values indicate the opposite. Dual: dualclassification system, CSF-NN: combined shape-based features and neural network; CSF-SVM, combined shape-based features and support vector machine. 


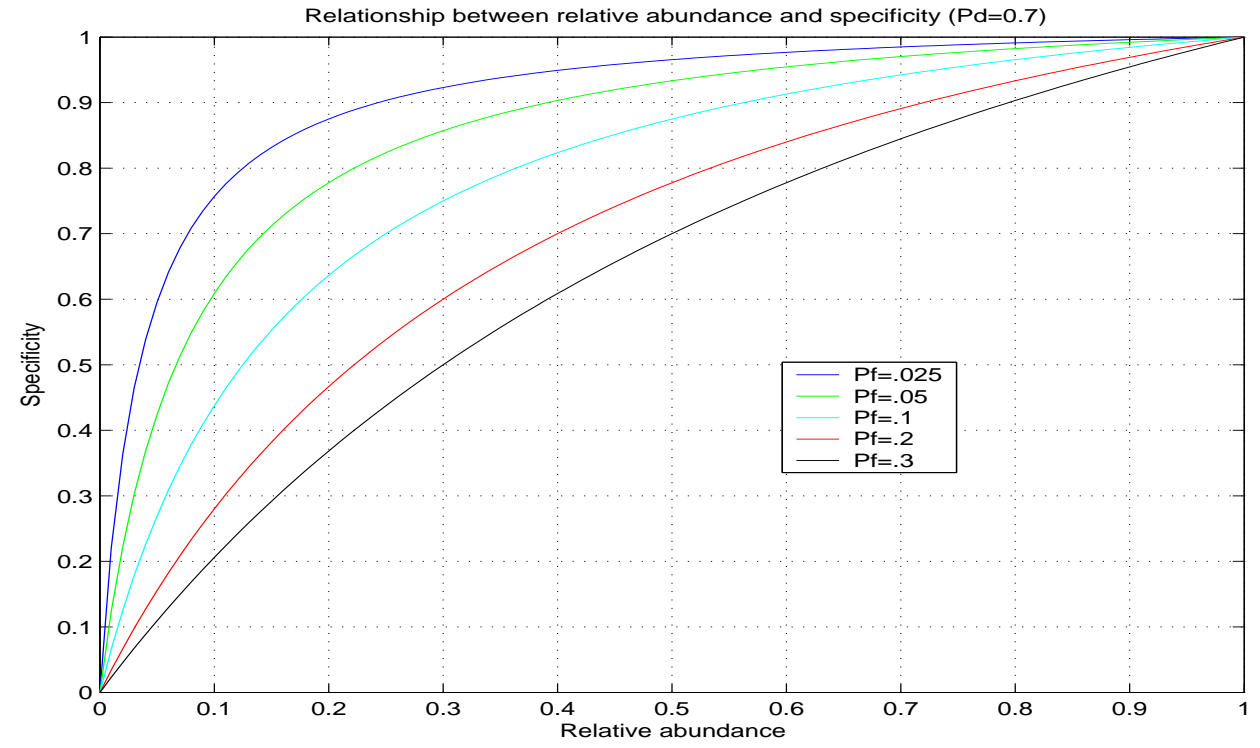

Figure 6-6: Relationship between specificity and relative abundance for different false alarm rates. Probability of detection is set to $70 \%$. As false alarm rates become smaller, the range in which the specificity closes to a constant becomes wider. The dual-classification system has substantially reduced the false alarm rates, so that the specificity of each taxon in the whole study area is close to a constant. This makes fully automatic correction possible. 
The difficulty of general object classification may be overlooked because humans are so good at visual classification of objects. We take for granted our ability to identify facial images without considering the millions of years of evolution involved. On the other hand, a computer is taught in less than 1 hour to identify plankton images that suffer from projection variance, occlusion, non-uniform illumination, and noise, using 200 training images per taxon. The assessment study in Chapter 3 [34] revealed the difficulty level of this data set. I showed that the $90 \%+$ accuracy on a selected subset of these data [150] only yielded $60 \%$ accuracy on the entire data set. Although humans are able to identify some of the images in this data set to a higher level of taxonomic group, the dual-classification method presented in this chapter yields abundance estimation almost as accurate as those of human experts.

\subsection{Conclusion}

In this chapter, I used a dual-classification system by building an SVM classifier on texture-based features (co-occurrence matrices) and an LVQ-NN classifier on shapebased features (moment invariants, Fourier descriptors and granulometry) to jointly identify over 20,000 VPR images. A confusion matrix was built from training samples. Sensitivity and specificity of the classification system were calculated from the confusion matrix to correct the abundance estimation. After correction, the dualclassification system reliably estimated the abundance of a taxon even when its relative abundance was as low as $2.5 \%$. In regions of relatively low abundance, the dual-classification system reduced the abundance estimation error by 50 to $100 \%$ compared with previous methods. Because it is fully automatic, this method can be used for real-time applications, as our previous methods discussed in Chapter 3 and $5[34,73]$. 


\section{Chapter 7}

\section{Conclusions and future work}

The ultimate goal of this thesis is to build an automatic classification system which can automatically obtain fine-scale abundance estimation of different taxonomic groups from the Video Plankton Recorder. A statistical machine learning approach was used to train the classification system. The major contributions of this thesis include constructing a large real-world labeled data set, developing real-time focus detection algorithms and evaluating their performance on the recorded VPR video, investigating different pattern representation methods on this large data set, assessing an existing learning vector quantization neural network classification system on this data set in a systematic way, extracting features from multi-scale co-occurrence matrices, designing different classification schemes, and proposing different correction methods to correct classification results.

This is the first study of taxa-specific abundance estimation with machine learning and pattern recognition on field-collected images from plankton imaging sampler. It is the first study to compare the classification systems on a such large data set which includes all the samples collected from the Video Plankton Recorder. By using dualclassification system and automatic correction method, the abundance estimation is almost as good as that of manually classified results. The findings in this thesis can be applied to researches which have similiar problems, i.e., projection variance and occlusion. The classification assessment will provides the guidance of model selection and parameter estimation. The multi-scale texture-based features should be used 
in these applications. In order to make the dual-classification work, the quality of images need to have information about both shape and texture. In other words, both of the classifiers in the system should have relative high of probability of detection.

\subsection{Summary of major contributions}

\section{Focus detection}

Three different real-time focus detection algorithms were developed and calibrated from four video sections. This was the first quantitative study of real-time focus detection algorithms of the Video Plankton Recorder. The performance of the algorithms was good in terms of both probability of detection and probability of false alarm. Special care was needed in the extremely high abundant regions. The problem can be corrected with careful calibration of the focus detection program.

\section{Feature representation}

A group of most commonly used texture-based features and shape-based features was compared on a random set of real world field-collected VPR images. This study demonstrated that texture-based features were more important than shape-based features in classifying field-collected images due to the non-linear illumination, occlusion and project variance. Among all the feature representation methods, features from multi-scale co-occurrence matrices were the best. The mean classification accuracy was $73 \%$ for seven taxa on independent testing data set.

\section{Feature extraction and selection}

Multi-scale co-occurrence matrices were designed with 4 different angles and 4 different separation distances. Mean and range matrices from each separation distance were used to achieve relative rotation invariance. Normalization was also applied to the resulting matrices to achieve scale invariance. The angular second moment (energy), contrast, correction, variance, inverse-different moment, entropy, sum entropy, 
and difference entropy of these matrices were calculated and used as feature vector. Because these features were less sensitive to occlusion and projection, a support vector machine trained on these features reduced the classification error rate from 39 to $28 \%$, compared to a previous plankton recognition system using a combined shape-based features.

\section{Classifier design}

Three different classifier designs were implemented and tested on the data set. First, a two-pass classification system was implemented based on a learning vector quantization neural network classifier. The main idea of this approach is to estimate the local priors of each taxon recursively. In the first classification, the uniform prior was used. The classification was based on maximizing likelihood of feature vectors. The result of the first classifier was used to estimate the priors of each taxon. In the second classification, these priors as well as feature vectors were used to maximize a posteriori. This scheme can be extended to n-pass classification system. For simplicity, a two-pass classification system was implemented and tested.

Second, a distance rejection metric was developed on a learning vector quantization neural network classifier. After a classifier was trained, each training sample was classified by the classifier. The mean and standard distances between a correctly classified training sample and winning neuron were computed. A distance limit was calculated from these two values for each taxon. During the classification phase, an extra step was performed. The distance between the winning neuron and the sample being classified was compared to the distance limit of corresponding taxon. If the distance was less than the distance limit, the sample was classified as same taxon as the winning neuron. Otherwise, it was classified as "unknown".

At the end, I developed a dual-classification system by taking advantage of both shape-based features and texture-based features, as well as a learning vector quantization neural network classifier and a support vector machine classifier. One of the problems to limit the accuracy of abundance estimation is the relative large probability of false alarm. To overcome this problem, I proposed a dual-classification system. 
In the training phase, an LVQ-NN classifier based on shape-based features and an SVM based on texture-based features were trained in parallel. In the classification phase, both classifiers were used to predict the label of the sample independently. A classifier committee was called to see if both classifiers agreed on the label. If this was the case, the sample was classified as the label that both classifiers were agreed on. Otherwise, the sample was classified as "unknown".

\section{Abundance estimation}

The ultimate goal of this thesis is to obtain accurate and reliable species-specific abundance estimation from the video images. To this end, I proposed 3 different methods to get better abundance estimation from classification with correction. A statistical correction method was applied directly on the classified results. The classifier was modeled as probabilistic and was characterized by the confusion matrix. This method traded bias with variance. It offered less bias but larger variance estimation. Due to the uncertainty of confusion matrix estimation, this method might estimate negative abundance in some locations.

An automatic correction method was developed to correct the dual-classification

results. Instead of using the confusion matrix itself, probability of detection and specificity of each taxon were calculated from the confusion matrix. These values were used to scale the abundance estimation accordingly. Except for one taxon, the automatically corrected abundance estimation was almost as good as that of a human expert manually going through all the images. It yielded perfect abundance estimation for less abundance taxon which made up $2.5 \%$ of total abundance.

A correction method was developed to correct a single classification result with manual correction. This method only utilized the probability of detection from confusion matrix. A human expert needs to go through the classified results to pull out the false positives. The scaling factor was similar to automatic correction method with all the specificities being unity. 


\subsection{Future research directions}

In this thesis, I developed a classification system which could reliably estimate the abundance of major planktonic taxa in real time. However, the accuracy of each individual identification was still far below human identification despite significant improvement from previous systems. The limitation on machine accuracy was partly due to the image quality. In order to further shorten the gap between human and machine identification, new sensors are needed to overcome some difficulties associated with field sampling. Two directions are promising for futther exploration, namely 3-D plankton recognition and colored plankton image recognition.

\section{D plankton recognition}

One major hindrance to identifying zooplankton accurately in 2-D images is the projection variation. A copepod looks very different in shape from different view points. If a 3-D imaging system is applied, the projection variance will be no longer exist. The object can be rotated or oriented to a certain attitudes which makes the object easy to identify.

One such system is computational digital holography. The digital in-line holography is able to record $150 \mathrm{ml}$ image volume on a CCD and reconstruct sub-millimeter resolution slices in the axial direction.

Most of the feature representation methods used in this thesis have a natural extension to 3-D. For example, moment invariants, co-occurrence matrices, and granulometry can be easily extended to 3-D. Fourier descriptors have no such extension. However, three principal axes can be computed from a 3-D image and outlines of the object in cross-section along these axes can be encoded with Fourier descriptors.

One of the challenges to using such a system is how to quickly reconstruct all the slices in the image volume and pick out all the regions of interest. Likewise, the feature extraction time and disk requirements will be increased accordingly. 


\section{Colored plankton image recognition}

Another challenge of in situ sampling is occlusion. Since we have no control over the samples that we are sampling, part of the object we are sampling may be out of the image volume. Furthermore, nonlinear illumination can also cause occlusion problem during segmentation. I demonstrated that using texture-based features could overcome part of this problem. Texture is not the only feature which is not sensitive to occlusion. The other feature which has such a characteristic is color.

Plankton have color, at least to a certain degree. Color provides independent features of plankton besides shape and texture. Color can either be combined with other features (shape and texture) or be used alone as a classifier component. The first approach will yield a more overall accurate classification system, while the second approach may significantly reduce the false alarm rate such that the classification system will obtain reliable abundance estimation on extremely low abundant species.

Color invariants are well studied. Color angles are commonly used color invariants. The existing technologies can greatly shorten the developing time. 


\section{Bibliography}

[1] D. L. Aksnes and T. Magnesen. Distribution, development, and production of calanus finmarchicus(Gunnerus) in Lindaspollene, Western Norway 1979. Sarsia, 68:195-207, 1983.

[2] D. L. Aksnes, C. B. Miller, M. D. Ohman, and S. N. Wood. Estimation techniques used in studies of copepod population dynamics - a review of underling assumptions. Sarsia, 82:279-296, 1997.

[3] E. L. Allwein, R. E. Schapire, and Y Singer. Reducing multiclass to binary: a unifying approach for margin classfiers. Journal of Machine Learning Research, 1:113-141, 2001.

[4] C. Apstein. Das Süsswasserplankton Kiel und Leipzig. Lipsius and Tescher, Kiel, 1896.

[5] C. J. Ashjian, C. S. Davis, S. M. Gallager, and P. Alatalo. Distribution of plankton, particles and hydrographic features across Georges Bank described using the Video Plankton Recorder. Deep-Sea Research II, 48:245-282, 2001.

[6] P. Baldi and S. Brunak. Bioinformatrics - the machine learning approach. MIT Press, Cambridge, MA, 2001.

[7] S. O. Belkasim, M. Shridhar, and A. Ahmadi. Pattern recognition with moment invariants: A comparative study and new results. Pattern Recognition, 42:11171138, 1991. 
[8] R. Bellman. Adaptive Control Processes: A Guided Tour. Princeton University Press, 1961.

[9] M. C. Benfield, C. S. Davis, P. H. Wiebe, R. G. Lough, S. M. Gallager, and N. J. Copley. Video Plankton Recorder estimates of copepod, pteropod and larvacean distributions from a stratified region of Georges Bank with comparative measurements from a MOCNESS sampler. Deep-Sea Research II, 43:1925-1945, 1996.

[10] M. C. Benfield, C.J. Schwehm, R.G. Fredericks, G. Squyresand S.F. Keenan, and M.V. Trevorrow. ZOOVIS: A high-resolution digital still camera system for measurement of fine-scale zooplankton distributions. In P. Strutton and L. Seuront, editors, Scales in Aquatic Ecology: Measurement, Analysis and Simulation. CRC Press, 2003.

[11] A. Del Bimbo and P. Pala. Visual image retrieval by elastic matching of user sketches. IEEE Trans. on Pattern Analysis and Machine Intelligence, 19:121$132,1997$.

[12] L. Boddy and C. W. Morris. Analysis of flow cytometry data - a neural network approach. Binary - Computing in microbiology, 5:17-22, 1993.

[13] O. Bousquet and A. Elisseeff. Stability and generalization. Journal of Machine Learning Research, 2:499-526, 2002.

[14] M. Brown and D. Lowe. Invariant features from interest point groups. In British Machine Vision Conference, pages 656-665, Cardiff Wales, 2002.

[15] C. J. C. Burges. A Tutorial on Support Vector Machines for Pattern Recognition. Data Mining and Knowledge Discovery, 2:121-167, 1998.

[16] D. J. Burr. Elastic matching of line drawings. IEEE Trans. on Pattern Analysis and Machine Intelligence, 3:708-713, 1981. 
[17] F. Carlotti, J. Giske, and F. Werner. Modeling zooplankton dynamics. In R. Harris, P. Wiebe, J. Lenz, H. R. Skjoldal, and M. Huntly, editors, ICES Zooplankton Methodology Manual, pages 533-570, London, 2000. Academic Press.

[18] D. Casasent and D. Psaltis. Position, rotation and scale invariant optical correlation. Applied Optics, 15:1795-1799, 1976.

[19] D. Casasent and D. Psaltis. New optical transforms for pattern recognition. Proceedings of the IEEE, 65:77-84, 1977.

[20] T. Chang and C.-C. J. Kuo. Texture analysis and classification with treestructured wavelet transform. IEEE Trans. on Image Processing, 2:429-441, 1993.

[21] Y. Q. Chen, M. S. Nixon, and D. W. Thomas. Statistical geometrical features for texture classification. Pattern Recognition, 28:537-552, 1995.

[22] A. Chu, C. M. Sehgal, and J. F. Greenleaf. Use of gray value distribution of run lengths for texture analysis. Pattern Recognition Letters, 11:415-419, 1990.

[23] M. Clark and A. C. Bovik. Texture segmentation using Gabor modulation/demodulation. Pattern Recognition Letters, 6:261-267, 1987.

[24] G. L. Clarke and D. F. Bumpus. The plankton sampler - an instrument for quantitative plankton investigations. Special Publication of the American Society of Limnology and Oceanography, 5:1-8, 1950.

[25] E. Costas, M. I. San Andres, S. Gonzalas-Gil, A. Aguilera, and V. LopezRodas. A procedure to estimate okadaic acid in whole dinoflagellate cells using immunological technigues. Journal of Applied Phycology, 7:407-411, 1995.

[26] K. Crammer and Y. Singer. On the learnability and design of output codes for multiclass problems. Machine Learning, 47:201-233, 2002. 
[27] P.F. Culverhouse, R. E. Ellis, R. Simpson, R. Williams, R. W. Pierce, and J. T. Turner. Categorisation of 5 species of cymatocylis (tintinidae) by artificial neural network. Marine Ecology Progress Series, 107:273-280, 1994.

[28] P.F. Culverhouse, R. Williams, B. Reguera, V. Herry, and S. González-Gil. Do experts make mistakes? a comparison of human and machine identification of dinoflagellates. Marine Ecology Progress Series, 247:17-25, 2003.

[29] B. V. Dasarathy and E. B. Holder. Image characterizations based on joint gray level run length distributions. Pattern Recognition Letters, 12:497-502, 1991.

[30] J.G. Daugman. Uncertainty relation for resolution in space, spatial frequency, and orientation optimized by two-dimensional visual cortical filters. osa, 2:11601169, 1985.

[31] C. S. Davis, S. M. Gallager, M. S. Berman, L. R. Haury, and J. R. Strickler. The Video Plankton Recorder (VPR): Design and initial result. Archiv. Hydrobiol. Beih., 36:67-81, 1992.

[32] C. S. Davis and S. M. Gallager A. R. Solow. Microaggregations of oceanic plankton observed by towed video microscopy. Science, 257:230-232, 1992.

[33] C.S. Davis, S. M. Gallager, M. Marra, and W. K. Stewart. Rapid visualization of plankton abundance and taxonomic composition using the Video Plankton Recorder. Deep-Sea Research II, 43:1947-1970, 1996.

[34] C.S. Davis, Q. Hu, S. M. Gallager, X. Tang, and C. J. Ashjian. Real-time observation of taxa-specifc plankton distribution: an optical sampling method. Marine Ecology Progress Series, 287:77-96, 2004.

[35] C.S. Davis, F. Thwaite, S. M. Gallager, and Q. Hu. A three-axis fast-tow digital Video Plankton Recorder for rapid surveys of plankton taxa and hydrography. Limnology Oceanography Method, 3:59-74, 2005. 
[36] J. L. de Bougrenet de la Tocnaye and F. Ghorbel. A rotation, scaling and translation invariant pattern classification system. Pattern Recognition Letters, 8:55-58, 1988.

[37] D. Decoste and B. Schölkopf. Training invariant support vector machines. Machine Learning, 24:797-805, 2002.

[38] M. R. Dennett, D. A. Caron, A. F. Michaels, S. M. Gallager, and C. S. Davis. Video plankton recorder reveals high abundance of colonial radiolaria in surface waters of the Central North Pacific. Journal of Plankton Research, 24:797-805, 2002.

[39] T. G. Dietterich and G. Bakiri. Solving multiclass learning problems via errorcorrecting output codes. Journal of Artificial Intelligence Research, 2:263-286, 1995.

[40] C. Dorai and A. K. Jain. COSMOS - a representation scheme of 3d free-form objects. IEEE Trans. on Pattern Analysis and Machine Intelligence, 19:11151130, 1997.

[41] C. Dorai and A. K. Jain. Shape spectrum based view grouping and matching of 3d free-form objects. IEEE Trans. on Pattern Analysis and Machine Intelligence, 19:1139-1146, 1997.

[42] R. O. Duda, P. E. Hart, and D. G. Stork. Pattern Classification. John Wiley \& Sons, Inc, New York, 2001.

[43] A. Elisseeff, T. Evgeniou, and M. Pontil. Stability of randomized learning algorithms. Journal of Machine Learning Research, 6:55-79, 2005.

[44] T. Evgeniou, M. Pontil, and T. Poggio. Regularization networks and support vector machines. Advances in Computational Mathematics, 13:1-50, 2000.

[45] M. J. R. Fasham. The statistical and mathematical analysis of plankton pathiness. Oceanography and Marine Biology - An Annual Review, 16:43-79, 1978. 
[46] M. J. R. Fasham. Modelling the marine biota. In M. Heimann, editor, The global carbon cycle, pages 457-504. Springer-Verlag, Berlin, 1993.

[47] K. G. Foote and T. K. Stanton. Acoustical methods. In R. P. Harris, P. H. Wiebe, J. Lenz, H. R. Skjoldal, and M. Huntley, editors, ICES zooplankton methodology manual, pages 259-295. Academic Press, London, 2000.

[48] P. J. S. Franks and J. S. Jaffe. Microscale distributions of phytoplankton: initial results from a two-dimensional imaging fluorometer, osst. Marine Ecology Progress Series, 220:59-72, 2001.

[49] J. H. Fraser. Zooplankton sampling. Nature, 211:915-916, 1966.

[50] R. Froese, K. G. Barthel, W. Welsch, M. Rolke, C. Schubert, B. Hermann, S. Mees, D. Schnack, and J. Lenz. Development of an underwater video system for recording of ichthyoplankton and zooplankton. In ICES Council Meeting, ICES Biological Oceanography Communication, page 5pp. Copenhagen, Denmark, 1990.

[51] D. Gabor. Theory of communication. In Proceedings of the Institute of Electrical Engineers, volume 93, pages 429-457, 1946.

[52] M. M. Galloway. Texture analysis using gray level run lengths. Computer Graphics Image Processing, 4:172-179, 1975.

[53] D. Geiger, A. Gupta, L. Costa, and J. Vlontzos. Dynamic programming for detecting, tracking, and matching deformable contours. IEEE Trans. on Pattern Analysis and Machine Intelligence, 17:294-302, 1995.

[54] G. G. Gordon. Face recognition based on depth maps and surface curvature. In Proceedings of SPIE Geometric Methods in Computer Vision, San Diego, 1991.

[55] G. Gorsky, C. Aldorf, M. Kage, M. Picheral, Y. Garcia, and J. Favole. Vertical distribution of suspended aggregates determined by a new underwater video profiler. Annales de l'Institut Océanographique, 68:275-280, 1992. 
[56] J. Goutsias and D. Schonfeld. Morphological representation of discrete and binary images. IEEE Trans. on Signal Processing, 39:1369-1379, 1991.

[57] G. H. Granlund. Fourier preprocessing for hand print character recognition. IEEE Trans. on Computers, 21:195-201, 1972.

[58] P. Grosjean, M. Picheral, C. Warembourg, and G. Gorsky. Enumeration, measurement, and identification of net zooplankton samples using the ZOOSCAN digital image system. ICES Journal of Marine Science, 61:518-525, 2004.

[59] R. M. Haralick, K. Shanmugam, and I. Dinstein. Texture feature for image classification. IEEE Trans. Systems, Man, and Cybernetics, 3:610-621, 1973.

[60] A. C. Hardy. The Discovery Expedition: A new method of plankton research. Nature, 118:630-632, 1926.

[61] L. R. Haury, J. A. McGowan, and P. H. Wiebe. Patterns and processes in the time-space scales of plankton distributions. In J. H. Steele, editor, Spatial patterns in plankton communities, pages 277-327. Plenum Press, New York, 1978.

[62] L. R. Haury and P. H. Wiebe. Fine-scale multi-species aggregations of oceanic zooplankton. Deep-Sea Research I, 29:915-921, 1982.

[63] L. R. Haury, P. H. Wiebe, and S. H. Boyd. Longhurst-Hardy plankton recorders: their design and use to minimize bias. Deep-Sea Research I, 23:1217-1229, 1976.

[64] L. R. Haury, P. H. Wiebe, M. H. Orr, and M. G. Briscoe. Tidally generated highfrequency internal wave packets and their effect on plankton in Massachusetts Bay. Journal of Marine Research, 41:65-112, 1983.

[65] D. C. He and L. Wang. Texture features based on texture spectrum. Pattern Recognition, 25:391-399, 1991. 
[66] B. Heisele, T. Serre, M. Pontil, and T. Poggio. Component-based face detection. In IEEE Computer Society Conference on Computer Vision and Pattern Recognition(CVPR 2001), pages 657-662, Kauai Marriott Hawaii, 2001.

[67] V. Hensen. Über die Bestimmung des Planktons oder des im Meere treibenden Materials and Pflanzen und Thieren. Berichte der Kommssion wissenschaftlichen Untersuchung der deutschen Meere in Kiel, 5:1-107, 1895.

[68] A. W. Herman. Simultaneous measurement of zooplankton and light attenuance with a new optical plankton counter. Continental Shelf Research, 8:205-221, 1988.

[69] J. W. Hofstraat, W. J. M. van Zeijl, M. E. J. de Vreeze, J. C. H. Peeters, L. Peperzak, and F. Colijn T. W. M. Rademaker. Phytoplankton monitoring by flow cytometry. Journal of Plankton Research, 16:1197-1224, 1994.

[70] J. J. Hopfield. Neural networks and physical systems with emergent collective computational abilities. Proc. Natl. Acad. Sci. USA, 79:2554-2558, 1982.

[71] W. E. Hoyle. On a deep sea tow-net for opening and shutting under water. Proceedings and Transactions of the Liverpool Biological Society, 3:100-112, 1889.

[72] M.-K. Hu. Visual pattern recognition by moment invariants. IRE Trans. Information Theory, 8:179-187, 1962.

[73] Q. Hu and C. Davis. Automatic plankton image recognition with co-occurrence matrices and support vector machine. Marine Ecology Progress Series, 295:21$31,2005$.

[74] Q. Hu and C. Davis. Accurate automatic quantification of taxa-specific plankton abundance using dual classification with correction. Marine Ecology Progress Series, 306, 2006. 
[75] J. S. Jaffe, M. D. Ohman, and A. D. Roberts. OASIS in the sea: measurement of the acoustic reflectivity of zooplankton with concurrent optical imaging. DeepSea Research II, 45:1239-1253, 1998.

[76] A. K. Jain. Dimensionality and sample size considerations in pattern recognition practice. In P. R. Krishnaiah and I. N. Kanal, editors, Handbook of Statistics, pages 835-855. North-Holland, Amsterdam, 1982.

[77] A. K. Jain, P. W. Duin, and J. Mao. Statistical pattern recognition: A review. IEEE Trans. on Pattern Analysis and Machine Intelligence, 22:4-37, 2000.

[78] A. K. Jain, S. Prabhakar, and Hong L. A multichannel approach to fingerprint classification. IEEE Trans. on Pattern Analysis and Machine Intelligence, 21:348-359, 1999.

[79] A. K. Jain, Y. Zhong, and M.-P. D. Dubuisson-Jolly. Deformable template models: A review. Signal Processing, 71:109-129, 1998.

[80] A. K. Jain and D. Zongker. Representation and recognition of handwritten digits using deformable templates. IEEE Trans. on Pattern Analysis and Machine Intelligence, 19:1386-1390, 1997.

[81] H. P. Jeffries, M. S. Berman, A. D. Poularikas, C. Katsinis, I. Melas, K. Sherman, and L. Bivins. Automated sizing, counting and identification of zooplankton by pattern recognition. Marine Biology, 78:329-334, 1984.

[82] H. P. Jeffries, K. Sherman, R. Maurer, and C. Katsinis. Computer processing of zooplankton samples. In V. Kennedy, editor, Estuarine perspectives, pages 303-316. Academic Press, New York, 1977.

[83] P. M. Jenkins. The methods and results of the German plankton investigations, with special reference to the Hensen Nets. Proceedings and Transactions of the Liverpool Biological Society, 15:279-341, 1901.

[84] I. T. Jolliffe. Principal Component Analysis. Springer-Verlag, 1986. 
[85] B. Julesz. Visual pattern discrimination. IRE Trans. Information Theory, 8:84$92,1962$.

[86] S. Kageyu, N. Ohnishi, and N. Sugie. Augmented multilayer perceptron for rotation- and scale-invariant handwritten numerical recognition. In Proceedings of IEEE International Joint Conference On Neural Networks, volume 1, pages 54-59, 1991.

[87] M. Kass, A. Witkin, and D. Terzopoulos. Snake: active contour models. International Journal of Computer Vision, 1:321-331, 1988.

[88] J. Katz, P. L. Donaghay, J. Zhang, S. King, and K. Russell. Submersible holocamera for detection of particle characteristics and motions in the ocean. Deep-Sea Research I, 46:1455-1481, 1999.

[89] H. Kauppinen, T. Seppanen, and M. Pietikainen. An experimental comparison of autoregressive and fourier based descriptors in $2 \mathrm{~d}$ shape classification. IEEE Trans. on Pattern Analysis and Machine Intelligence, 17:201-207, 1995.

[90] A. Khotanzad and Y. H. Hong. Invariant image recognition by Zernike moments. IEEE Trans. on Pattern Analysis and Machine Intelligence, 12:489-497, 1990.

[91] A. Khotanzad and Y. H. Hong. Rotation invariant image recognition using features selected via a systematic method. Pattern Recognition, 23:1089-1101, 1990.

[92] H. Kim, P. Howland, and H. Park. Dimension reduction in text classification with support vector machines. Journal of Machine Learning Research, 6:37-53, 2005.

[93] H. S. Kim and H.-K. Lee. Invariant image watermark using zernike moments. IEEE Transactions on Circuts and Systems for Video Technology, 13:766-775, 2003. 
[94] F. P. Kuhl and C. R. Giardina. Elliptic fourier features of a closed contour. Computer Vision, Graphics and Image Processing, 18:236-258, 1982.

[95] K. I. Law. Textured Image Segmentation. PhD dissertation, University of Southern California, 1980.

[96] B. B. Leavitt. A quantitative study of the vertical distribution of the larger zooplankton in deep water. Biological Bulletin, 68:115-130, 1935.

[97] Y. LeCun, B. Boser, J. S. Denker, D. Henderson, R. E. Howard, W. Hubbard, and L. D. Jackel. Backpropagation applied to handwritten zip code recognition. Neural Computation, 1:541-551, 1989.

[98] Y. LeCun, L. Bottou, Y. Bengio, and P. Haffner. Gradient-based learning applied to document recognition. Proceedings of the IEEE, 86:2278-2324, 1998.

[99] J. Lenz, D. Schnack, D. Petersen, J. Kreikemeier, B. Hermann, S. Mees, and K. Wieland. The Ichthyoplankton Recorder: a video recording system for in situ studies of small-scale plankton distribution patterns. ICES Journal of Marine Science, 52:409-417, 1995.

[100] F. Leymarie and M. Levine. Tracking deformable objects in the plane using as active contour model. IEEE Trans. on Pattern Analysis and Machine Intelligence, 15:617-634, 1993.

[101] Y. Li. Reforming the theory of invariant moments for pattern recognition. Pattern Recognition Letters, 25:723-730, 1992.

[102] C.-S. Lin and C.-L. Huang. New forms of shape invariants from elliptic fourier descriptors. Pattern Recognition, 20:535-545, 1987.

[103] A. R. Longhurst, A. D. Reith, R. E. Bower, and D. L. R. Seibert. A new system for the collection of multiple serial plankton samples. Deep-Sea Research I, $13: 213-222,1966$. 
[104] D. Lowe. Object recognition from local scale-invariant features. In International Conference on Computer Vision, pages 1150-1157, Corfu Greece, 1999.

[105] D. Lowe and A. R. Webb. Optimized feature extration and the bayes decision in feed-forward classifier networks. IEEE Trans. on Pattern Analysis and Machine Intelligence, 13:355-364, 1991.

[106] T. Luo, K. Kramer, D. B. Goldgof, L. O. Hall, S. Samson, A. Remsen, and T. Hopkins. Recognizing plankton images from the shadow image particle profiling evaluation recorder. IEEE Trans. Systems, Man, and Cybernetics Part B - Cybernetics, 34:1753-1762, 2004.

[107] D. R. Lynch, W. C. Gentleman, D. J. Jr McGillicuddy, and C. S. Davis. Biological/physical simulations of calanus finmarchicus population dynamics in the Gulf and Maine. Marine Ecology Progress Series, 169:189-210, 1998.

[108] D. L. Mackas, K. L. Deman, and M. R. Abbott. Plankton pathiness: Biology in the physical vernacular. Bulletin of Marine Science, 37:652-674, 1985.

[109] V. Manian, R. Vasquez, and P. Katiyar. Texture classification using logical operators. IEEE Trans. on Image Analysis, 9:1693-1703, 2000.

[110] J. Mao and A. K. Jain. Artificial neural networks for feature extraction and multivariate data projection. IEEE Trans. on Neural Networks, 6:296-317, 1995.

[111] P. Maragos. Pattern spectrum and multiscale shape representation. IEEE Trans. on Pattern Analysis and Machine Intelligence, 11:701-716, 1989.

[112] G. Matheron. Random Sets and Integral Geometry. Wiley, New York, 1975.

[113] W. S. McCulloch and W. Pitts. A logical calculus of ideas immanent in nervous activity. Bull. Mathematical Biophysics, 5:115-133, 1943. 
[114] A. Meijster and M. H. F. Wilkinson. A comparison of algorithms for connected set openings and closings. IEEE Trans. on Pattern Analysis and Machine Intelligence, 24:484-494, 2002.

[115] C. B. Miller, D. R. Lynch, F. Carlotti, W. C. Gentleman, and C. V. W. Lewis. Coupling of an individual-based population dynamic model of calanus finmarchicus to a circulation model for the Geoges Bank region. Fisheries Oceanography, 7:219-234, 1998.

[116] M. Minsky and S. Papert. Perceptrons: An Introduction to Computational Geometry. MIT Press, Cambridge Mass., 1969.

[117] T. M. Mitchell, R. Hutchinson, R. S. Niculescu, F. Pereira, and X. Wang. Learning to decode cognitive states from brain. Machine Learning, 57:145-175, 2004.

[118] A. Mohan, C. Papageorgiou, and T. Poggio. Example-based object detection in images by components. IEEE Trans. on Pattern Analysis and Machine Intelligence, 23:349-361, 2001.

[119] C. Nastar and M. Mitschke. Real-time face recognition using feature combination. In Proceedings of the third IEEE International Conference on Automatic Face and Gesture Recognition (FG'98), Nara Japan, 1998.

[120] M. Omori and T. Ikeda. Methods in Marine Zooplankton Ecology. John Wiley and Son, New York, 1984.

[121] N. Otsu. A threshold selection method from gray level histograms. IEEE Trans. Systems, Man, and Cybernetics, 9:62-66, 1979.

[122] A. Passerini, M. Pontil, and P. Frasconi. New results on error correcting output codes of kernel machines. IEEE Trans. on Neural Networks, 15:45-54, 2004.

[123] E. Persoon and K.-S. Fu. Shape discrimination using fourier descriptors. IEEE Trans. Systems, Man, and Cybernetics, 7:170-179, 1977. 
[124] F. Phan, E. Micheli-Tzanakou, and S. Sideman. Speaker identification using neural networks and wavelets. IEEE Engineering in Medicine and Biology, 19:92-101, 2000.

[125] M. Porat and Y.Y. Zeevi. The generalized gabor scheme of image representation in biological and machine vision. IEEE Trans. on Pattern Analysis and Machine Intelligence, 10:452-468, 1988.

[126] J. A. Posgay and R. R. Marak. The MARMAP bongo zooplankton samplers. Journal of Northwest Atlantic Fisheries Science, 1:91-99, 1980.

[127] W. K. Pratt. Digital Image Processing. John Wiley and Son, New York, 1991.

[128] C.-M. Pun and M.-C. Lee. Rotation-invariant texture classification using a twostage wavelet packet feature approach. In IEE Proceedings of Vision, Image and Signal Processing, volume 148, pages 422-428, 2001.

[129] S. J. Raudys and A. K. Jain. Small sample size effects in statistical pattern recognition: Recommendations for practioners. IEEE Trans. on Pattern Analysis and Machine Intelligence, 13:252-264, 1991.

[130] S. J. Raudys and V. Pikelis. On dimensionality, sample size, classification error, and complexity of classification algorithms in pattern recognition. IEEE Trans. on Pattern Analysis and Machine Intelligence, 2:243-251, 1980.

[131] T. H. Reiss. The revised fundamental theorem of moment invariants. IEEE Trans. on Pattern Analysis and Machine Intelligence, 13:976-986, 1991.

[132] B. Rieger and L. J. van Vliet. Curvature of n-dimensional space curves in grey-value images. IEEE Trans. on Image Processing, 11:738-745, 2002.

[133] R. Rifkin and A. Klautan. In defense of one-vs-all classification. Journal of Machine Learning Research, 5:101-141, 2004. 
[134] G. A. Riley. Theory of food chain relations in the ocean. In M. N. Hill, editor, The sea: the composition of sea water: comparative and descriptive oceanography, pages 438-463. Wiley, New York, 1963.

[135] M. Rolke and J. Lenz. Size structure analysis of zooplankton samples by means of an automated image analyzing system. Journal of Plankton Research, 6:637$645,1984$.

[136] R. Rosenblatt. Principles of Neurodynamics. Spartan Books, New York, 1962.

[137] D. E. Rumelhart and J. L. McClelland. Parallel Distributed Processing: Exploration in the Microstructure of Cognition. MIT Press, Cambridge Mass., 1986.

[138] S. Samson, T. Hopkins, A. Remsen, L. Langebrake, T. Sutten, and J. Patten. A system for high-resolution zooplankton imaging. IEEE Journal of Oceanic Engineering, 26:671-676, 2001.

[139] B. Schölkopf and A. J. Smola. Learning with Kernels. MIT press, Cambridge, Massachusetts, 2002.

[140] J. Serra. Image Analysis and Mathematical Morphology. Academic Press, London, 1982.

[141] Y. Sheng and H. H. Arsenault. Experiments on pattern recognition using invariant fourier-mellin descriptors. Journal of the Optical Society of America. A, Optics, image science, and vision, 3:771-776, 1986.

[142] Y. Sheng and J. Duvernoy. Circular fourier radial mellin transform descriptors for pattern recognition. Journal of the Optical Society of America. A, Optics, image science, and vision, 3:885-888, 1986.

[143] Y. Sheng and C. Lejeune. Invariant pattern recognition using fourier-mellin transforms and neural networks. Journal of Optics, 22:223-228, 1991. 
[144] C. K. Sieracki, M. E. Sieracki, and C. S. Yentsch. An imaging in-flow system for automated analysis of marine microplankton. Marine Ecology Progress Series, 168:285-296, 1998.

[145] R. Simpson, P. F. Culverhouse, R. Ellis, and R. Williams. Classification of euceratium gran. in neural networks. In IEEE International Conference on Neural Network in Ocean Engineering, pages 223-230, Wahington DC, 1991.

[146] A. Solow, C. Davis, and Q. Hu. Estimating the txaonomic composition of a sample when individuals are classified with error. Marine Ecology Progress Series, 216:309-311, 2001.

[147] J. R. Strickler and J. S. Hwang. Matched spatial filters in long working distance microscopy of phase object. In P. C. Cheng, P. P. Hwang, J. Wu, G. Wang, and H. Kim, editors, Focus on modern microscopy, pages 217-239. World Scientific, New Jersey, 2000.

[148] X. Tang. Transform Texture Classification. PhD dissertation, Massachusetts Institute of Technology and Woods Hole Oceanographic Institution, 1996.

[149] X. Tang. Multiple competitive learning network fusion for object classification. IEEE Trans. Systems, Man, and Cybernetics, 28:532-543, 1998.

[150] X. Tang, W. K. Stewart, L. Vicent, H. Huang, M. Marra, S. M. Gallager, and C. S. Davis. Automatic plankton image recognition. Artificial Intelligence Review, 12:177-199, 1998.

[151] C.-H. Teh and R. T. Chin. On image analysis by the methods of moments. IEEE Trans. on Pattern Analysis and Machine Intelligence, 10:496-513, 1988.

[152] P. Tiselius. An in situ video camera for plankton studies: design and preliminary observations. Marine Ecology Progress Series, 164:293-299, 1998.

[153] Fumiaki Tomita and Saburo Tsuji. Computer Analysis of Visual Textures. Kluwer Academic Publishers, Norwell, MA, USA, 1990. 
[154] L. Toth and P. F. Culverhouse. Three-dimensional object categorisation from static $2 \mathrm{~d}$ views using multiple coarse channels. Image and Vision Computing, 17:845-858, 1999.

[155] A. Tsai, A. Yezzi, Jr. W. Wells, C. Tempany, D. Tucker, A. Fan, W. E. Grimson, and A. Willsky. A shape-based approach to the segmentation medical imagery using level sets. IEEE Trans. on Medical Imaging, 22:137-154, 2003.

[156] Mihran Tuceryan and Anil K. Jain. Texture analysis. In C. H. Chen, L. F. Pau, and P. S. P. Wang, editors, The Handbook of Pattern Recognition and Computer Vision, part 2.1, pages 207-248. World Scientific Publishing Co., second edition, 1998.

[157] M. R. Turner. Texture discrimination by gabor functions. Biological Cybernetics, 55:71-82, 1986.

[158] M. Unser. Texture classification and segmentation using wavelet frames. IEEE Trans. on Image Processing, 4:1549-1560, 1995.

[159] V. N. Vapnik. Estimation of Dependences Based on Empirical Data. SpringerVerlag, Berlin, 1982.

[160] V. N. Vapnik. The Nature of Statistical Learning. Springer, New York, 1995.

[161] V. N. Vapnik. Statistical Learning Theory. Wiley, New York, 1998.

[162] L. Vincent. Morphological grayscale reconstruction in image analysis: application and efficient algorithm. IEEE Trans. on Image Processing, 2:176-201, 1993.

[163] L. Wang and G. Healey. Using zernike moments for illumination and geometry invariant classification of multispectral texture. IEEE Trans. on Image Processing, 7:196-203, 1998.

[164] A. J. Warner and G. C. Hays. Sampling by the Continuous Plankton Recorder survey. Progress in Oceanography, 34:237-256, 1994. 
[165] S. Watanabe. Pattern Recognition: Human and Mechanical. Wiley, New York, 1985.

[166] J. Watson, S. Alexander, G. Craig, D. C. Hendry, P. R. Hobson, R. S. Lampitt, J. M. Marteau, H. Nareid, M. A. Player, K. Saw, and K. Tipping. Simultaneous in-line and off-axis subsea holographic recording of plankton and other marine particles. Measurement Science and Technology, 12:L9-L15, 2001.

[167] P. Werbos. Beyond Regression: New Tools for Prediction and Analysis in the Behavioral Science. PhD dissertation, Harvard University, Cambridge Mass., 1974.

[168] P. H. Wiebe and M. C. Benfield. From the Hensen net toward four-dimensional biological oceanography. Progress in Oceanography, 56:7-136, 2003.

[169] P. H. Wiebe, K. H. Burt, S. H. Boyd, and A. W. Morton. A multiple opening/closing net and environmental sensing system for sampling zooplankton. Journal of Marine Research, 34:313-326, 1976.

[170] P. H. Wiebe and C. H. Greence. The use of high frequency acoustics in the study of zooplankton spatial and temporal patterns. In Proceedings of the NIPR Symposium on Polar Biology, volume 7, pages 133-157, Tokyo, 1994. National Institute of Polar Research.

[171] P. H. Wiebe and W. R. Holland. Plankton pathiness: effect of repeated net tows. Limnology and Oceanography, 13:315-321, 1968.

[172] P. H. Wiebe, A. W. Morton, A. M. Bradley, R. H. Backus, J. E. Craddock, V. Barber, T. J. Cowles, and G. R. Flierl. New developments in the mocness, an apparatus for sampling zooplankton and micronekton. Marine Biology, 87:313$323,1985$.

[173] P. H. Wiebe, T. K. Stanton, C. H. Greence, M. C. Benfield, H. M. Sosik, T. Austin, J. D. Warren, and T Hammar. BIOMAPER II: an integrated in- 
strument platform for coupled biological and physical measurements in coastal and oceanic regimes. IEEE Journal of Oceanic Engineering, 27:700-716, 2002.

[174] M. K. Wilkins and L. Boddy C. W. Morris. Proportion estimation with confidence limits. Journal of Microbiological Methods, 43:55-64, 2000.

[175] C. T. Zahn and R. Z. Roskies. Fourier descriptor for plane closed curves. IEEE Trans. on Computers, 21:269-281, 1972.

[176] F. Zernike. Beugungstheorie des schneidenverfahrens und seiner verbesserten form, der phasenkontrastmethode. Physica, 1:689-704, 1934. 